\title{
Homolytic C-H and N-H Bond Dissociation Energies of Strained Organic Compounds
}

\author{
Yong Feng, Lei Liu,* Jin-Ti Wang, Su-Wen Zhao, Qing-Xiang Guo* \\ Department of Chemistry, University of Science and Technology of China, Hefei 230026, \\ China
}

\section{Supporting Information}

\section{Table of contents}

Table 1. The dependence of the $\mathrm{C}-\mathrm{H}$ BDE of $\mathrm{CH}_{4}$ on the $\mathrm{p} \%$ values. (S2)

Table 2. The dependence of the energy of $\mathrm{CH}_{2}=\mathrm{CH}-\mathrm{CH}_{2} \bullet$ radical on the H-C-C-C dihedron angle and spin. (S3)

The Cartesian coordinates $(\AA)$ and calculated Energies and the thermal corrections to Enthalpy of optimized structures by UB3LYP/6-31G(d) method. (S4-S78) 
Table 1. The dependence of the $\mathrm{C}-\mathrm{H} \mathrm{BDE}$ of $\mathrm{CH}_{4}$ on the $\mathrm{p} \%$ values.

\begin{tabular}{llcccc}
\hline angle & \multicolumn{1}{c}{$\mathrm{BDE}$} & \multicolumn{2}{c}{$\mathrm{CH}_{4}$} & \multicolumn{2}{c}{$\mathrm{CH}_{3}$} \\
& $(\mathrm{kcal} / \mathrm{mol})$ & $\mathrm{E}(\mathrm{a} . \mathrm{u})$. & $\mathrm{p} \%$ (parent) & $\mathrm{E}$ (a.u.) & $\mathrm{p} \%$ (radical) \\
\hline 90.0 & 89.73393 & -40.48129 & 0.888 & -39.83829 & 1.000 \\
91.0 & 91.84864 & -40.48465 & 0.881 & -39.83828 & 0.999 \\
92.0 & 93.9006 & -40.48788 & 0.874 & -39.83824 & 0.997 \\
93.0 & 95.89608 & -40.49100 & 0.867 & -39.83818 & 0.993 \\
94.0 & 97.82253 & -40.49397 & 0.860 & -39.83808 & 0.987 \\
95.0 & 99.67996 & -40.49681 & 0.852 & -39.83796 & 0.980 \\
96.0 & 101.47464 & -40.49950 & 0.845 & -39.83779 & 0.972 \\
97.0 & 103.20029 & -40.50204 & 0.838 & -39.83758 & 0.963 \\
98.0 & 104.8632 & -40.50443 & 0.834 & -39.83732 & 0.952 \\
99.0 & 106.4508 & -40.50664 & 0.823 & -39.83700 & 0.941 \\
100.0 & 107.98192 & -40.50869 & 0.816 & -39.83661 & 0.929 \\
101.0 & 109.43774 & -40.51055 & 0.809 & -39.83615 & 0.916 \\
102.0 & 110.83709 & -40.51223 & 0.802 & -39.83560 & 0.903 \\
103.0 & 112.18624 & -40.51373 & 0.794 & -39.83495 & 0.889 \\
104.0 & 113.46636 & -40.51502 & 0.787 & -39.83420 & 0.876 \\
105.0 & 114.70255 & -40.51612 & 0.780 & -39.83333 & 0.862 \\
106.0 & 115.876 & -40.51700 & 0.773 & -39.83234 & 0.848 \\
107.0 & 117.00551 & -40.51767 & 0.766 & -39.83121 & 0.834 \\
108.0 & 118.09111 & -40.51812 & 0.759 & -39.82993 & 0.820 \\
109.0 & 119.1516 & -40.51835 & 0.752 & -39.82847 & 0.806 \\
110.0 & 120.14306 & -40.51834 & 0.746 & -39.82688 & 0.792 \\
111.0 & 121.09688 & -40.51809 & 0.739 & -39.82511 & 0.779 \\
112.0 & 122.02559 & -40.51761 & 0.732 & -39.82315 & 0.766 \\
113.0 & 122.91038 & -40.51687 & 0.726 & -39.82100 & 0.754 \\
114.0 & 123.77635 & -40.51588 & 0.719 & -39.81863 & 0.741 \\
115.0 & 124.61721 & -40.51463 & 0.712 & -39.81604 & 0.729 \\
116.0 & 125.4267 & -40.51311 & 0.706 & -39.81323 & 0.718 \\
117.0 & 126.21736 & -40.51133 & 0.699 & -39.81019 & 0.707 \\
118.0 & 126.98292 & -40.50926 & 0.693 & -39.80690 & 0.696 \\
119.0 & 127.72339 & -40.50691 & 0.686 & -39.80337 & 0.685 \\
120.0 & 128.4513 & -40.50427 & 0.680 & -39.79957 & 0.675 \\
\hline & & & & &
\end{tabular}


Table 2. The dependence of the energy of $\mathrm{CH}_{2}=\mathrm{CH}-\mathrm{CH}_{2} \bullet$ radical on the $\mathrm{H}-\mathrm{C}-\mathrm{C}-\mathrm{C}$ dihedron angle and spin.

\begin{tabular}{ccc}
\hline Dihedron angle & HF Energy (a.u.) & spin \\
\hline 0.0 & -117.26035 & 0.703 \\
3.0 & -117.26025 & 0.703 \\
6.0 & -117.25996 & 0.706 \\
9.0 & -117.25946 & 0.711 \\
12.0 & -117.25878 & 0.717 \\
15.0 & -117.25791 & 0.726 \\
18.0 & -117.25686 & 0.736 \\
21.0 & -117.25565 & 0.747 \\
24.0 & -117.25429 & 0.761 \\
27.0 & -117.25278 & 0.776 \\
30.0 & -117.25115 & 0.792 \\
33.0 & -117.24942 & 0.810 \\
36.0 & -117.24760 & 0.829 \\
39.0 & -117.24573 & 0.846 \\
42.0 & -117.24381 & 0.867 \\
45.0 & -117.24189 & 0.889 \\
48.0 & -117.23998 & 0.911 \\
51.0 & -117.23811 & 0.933 \\
54.0 & -117.23631 & 0.954 \\
57.0 & -117.23461 & 0.975 \\
60.0 & -117.23301 & 0.994 \\
63.0 & -117.23155 & 1.012 \\
66.0 & -117.23024 & 1.028 \\
69.0 & -117.22908 & 1.042 \\
72.0 & -117.22808 & 1.054 \\
75.0 & -117.22724 & 1.064 \\
78.0 & -117.22656 & 1.072 \\
81.0 & -117.22604 & 1.078 \\
84.0 & -117.22567 & 1.082 \\
87.0 & -117.22545 & 1.085 \\
90.0 & -117.22538 & 1.086 \\
\hline & &
\end{tabular}


The Cartesian coordinates $(\AA)$ and calculated Energies and the thermal corrections to Enthalpy of optimized structures by UB3LYP/6-31G(d) method.

\section{Methane:}

\begin{tabular}{cccc}
\multicolumn{4}{c}{ Charge $=\mathbf{0}$ Multiplicity $=\mathbf{1}$} \\
C & -0.000001 & 0.000000 & 0.000044 \\
H & -0.000002 & 0.000001 & 1.093398 \\
H & 1.030701 & 0.000002 & -0.364552 \\
H & -0.515348 & -0.892611 & -0.364548 \\
H & -0.515347 & 0.892608 & -0.364560 \\
& & & \\
HF $=\mathbf{- 4 0 . 5 1 8 3 8 3 4}$ & &
\end{tabular}

Thermal correction to Enthalpy= 0.049033

\section{Methane $\mathrm{C1}$ radical:}

Charge $=0$ Multiplicity $=2$

$\begin{array}{lrrr}\text { C } & 0.000096 & -0.000167 & 0.000137 \\ \text { H } & -0.188686 & 0.326814 & 1.014966 \\ \text { H } & 0.906019 & 0.305882 & -0.507895 \\ \text { H } & -0.717911 & -0.631694 & -0.507895\end{array}$

$\mathrm{HF}=\mathbf{- 3 9 . 8 3 8 2 9 1 8}$

Thermal correction to Enthalpy $=\mathbf{0 . 0 3 3 8 8 1}$

Ethane:

Charge $=0$ Multiplicity $=1$

$\begin{array}{lrrr}\mathrm{C} & 0.000000 & 0.000000 & -0.665401 \\ \mathrm{C} & 0.000000 & 0.000000 & 0.665401 \\ \mathrm{H} & 0.923497 & 0.000000 & -1.239409 \\ \mathrm{H} & 0.923497 & 0.000000 & 1.239409 \\ \mathrm{H} & -0.923497 & 0.000000 & 1.239409 \\ \mathrm{H} & -0.923497 & 0.000000 & -1.239409\end{array}$

$\mathrm{HF}=\mathbf{- 7 8 . 5 8 7 4 5 8 2}$

Thermal correction to Enthalpy $=\mathbf{0 . 0 5 5 2 2 2}$

Ethane C1 radical:

Charge $=0$ Multiplicity $=2$

$\begin{array}{lrrr}\mathrm{C} & -0.001121 & 0.000000 & -0.724898 \\ \mathrm{C} & -0.087894 & 0.000000 & 0.582535 \\ \mathrm{H} & 0.781355 & 0.000000 & -1.474054 \\ \mathrm{H} & 0.799196 & 0.000000 & 1.226003 \\ \mathrm{H} & -1.046460 & 0.000000 & 1.102232\end{array}$


$\mathrm{HF}=\mathbf{- 7 7 . 9 0 1 2 0 8 2}$

Thermal correction to Enthalpy $=\mathbf{0 . 0 4 0 7 7 0}$

$i$-Propane:

Charge $=0$ Multiplicity $=1$

$\begin{array}{lrrr}\mathrm{C} & 1.000604 & 0.823151 & 0.144203 \\ \mathrm{H} & 1.034764 & 0.865222 & 1.239798 \\ \mathrm{H} & 2.033776 & 0.827882 & -0.222543 \\ \mathrm{C} & 0.238248 & -0.415649 & -0.337714 \\ \mathrm{H} & 0.251526 & -0.447998 & -1.435628 \\ \mathrm{H} & 0.762571 & -1.320525 & -0.001474 \\ \mathrm{C} & -1.211734 & -0.454730 & 0.155327 \\ \mathrm{H} & -1.735051 & -1.349336 & -0.202077 \\ \mathrm{H} & -1.256957 & -0.457925 & 1.251679 \\ \mathrm{H} & -1.772363 & 0.421340 & -0.194308 \\ \mathrm{H} & 0.519026 & 1.744714 & -0.206347\end{array}$

$\mathrm{HF}=\mathbf{- 1 1 9 . 1 4 4 2 4 8}$

Thermal correction to Enthalpy $=\mathbf{0 . 1 0 9 5 8 2}$

$i$-Propane $\mathrm{C} 2$ radical:

Charge $=0$ Multiplicity $=2$

$\begin{array}{lrrr}\mathrm{C} & 1.069699 & 0.751018 & 0.154722 \\ \mathrm{H} & 1.086886 & 0.722115 & 1.253530 \\ \mathrm{H} & 2.100018 & 0.643529 & -0.203255 \\ \mathrm{C} & 0.171433 & -0.300480 & -0.407180 \\ \mathrm{H} & 0.400322 & -0.705620 & -1.390445 \\ \mathrm{C} & -1.183823 & -0.550531 & 0.166411 \\ \mathrm{H} & -1.608127 & -1.498544 & -0.183594 \\ \mathrm{H} & -1.158925 & -0.574375 & 1.265193 \\ \mathrm{H} & -1.906386 & 0.241413 & -0.105942 \\ \mathrm{H} & 0.742360 & 1.771439 & -0.119206\end{array}$

$\mathrm{HF}=\mathbf{- 1 1 8 . 4 7 8 1 5 2 9}$

Thermal correction to Enthalpy $=0.094692$

$t$-Butane:

Charge $=0$ Multiplicity $=1$

$\begin{array}{lrrr}\mathrm{C} & 0.731406 & 1.266832 & 0.088717 \\ \mathrm{H} & 0.763966 & 1.323227 & 1.185000 \\ \mathrm{H} & 1.765644 & 1.285921 & -0.275647 \\ \mathrm{C} & -0.000816 & -0.001413 & -0.372520 \\ \mathrm{H} & -0.003361 & -0.005821 & -1.473087\end{array}$




$\begin{array}{rrrr}\mathrm{C} & 0.730944 & -1.265793 & 0.099390 \\ \mathrm{C} & -1.461680 & 0.000119 & 0.099390 \\ \mathrm{H} & 0.230640 & -2.173817 & -0.258272 \\ \mathrm{H} & 1.765760 & -1.287552 & -0.263004 \\ \mathrm{H} & 0.761562 & -1.313511 & 1.196037 \\ \mathrm{H} & -1.997901 & -0.887168 & -0.258272 \\ \mathrm{H} & -1.518315 & 0.002776 & 1.196037 \\ \mathrm{H} & -1.997932 & 0.885417 & -0.263004 \\ \mathrm{H} & 0.230818 & 2.172053 & -0.275647 \\ & & & \\ \text { HF }=\mathbf{- 1 5 8 . 4 5 8 8 1 4 1} & & \end{array}$

Thermal correction to Enthalpy= 0.139001

$t$-Butane $\mathrm{C} 2$ radical:

Charge $=0$ Multiplicity $=2$

$\begin{array}{lrrc}\mathrm{C} & 0.744619 & 1.289718 & 0.005934 \\ \mathrm{H} & 0.901326 & 1.561143 & 1.067873 \\ \mathrm{H} & 1.741624 & 1.239228 & -0.450164 \\ \mathrm{C} & -0.000379 & -0.000657 & -0.143765 \\ \mathrm{C} & 0.744507 & -1.289492 & 0.018872 \\ \mathrm{C} & -1.488987 & 0.000016 & 0.018872 \\ \mathrm{H} & 0.202691 & -2.131783 & -0.430194 \\ \mathrm{H} & 1.742030 & -1.243112 & -0.436527 \\ \mathrm{H} & 0.900028 & -1.551376 & 1.083207 \\ \mathrm{H} & -1.947524 & -0.890356 & -0.430194 \\ \mathrm{H} & -1.793545 & 0.003759 & 1.083207 \\ \mathrm{H} & -1.947582 & 0.887086 & -0.436527 \\ \mathrm{H} & 0.202390 & 2.127905 & -0.450164\end{array}$

$H F=\mathbf{- 1 5 7 . 7 9 8 3 2 7 4}$

Thermal correction to Enthalpy= 0.124604

Ethylene:

Charge $=0$ Multiplicity $=1$

$\begin{array}{lrrr}\mathrm{C} & 0.000000 & 0.000000 & -0.665401 \\ \mathrm{C} & 0.000000 & 0.000000 & 0.665401 \\ \mathrm{H} & 0.923497 & 0.000000 & -1.239409 \\ \mathrm{H} & 0.923497 & 0.000000 & 1.239409 \\ \mathrm{H} & -0.923497 & 0.000000 & 1.239409 \\ \mathrm{H} & -0.923497 & 0.000000 & -1.239409\end{array}$

$\mathrm{HF}=\mathbf{- 7 8 . 5 8 7 4 5 8 2}$

Thermal correction to Enthalpy $=\mathbf{0 . 0 5 5 2 2 2}$ 
Ethylene C1 radical:

Charge $=0$ Multiplicity $=2$

$\begin{array}{cccc}\mathrm{C} & -0.001121 & 0.000000 & -0.724898 \\ \mathrm{C} & -0.087894 & 0.000000 & 0.582535 \\ \mathrm{H} & 0.781355 & 0.000000 & -1.474054 \\ \mathrm{H} & 0.799196 & 0.000000 & 1.226003 \\ \mathrm{H} & -1.046460 & 0.000000 & 1.102232\end{array}$

$\mathrm{HF}=\mathbf{- 7 7 . 9 0 1 2 0 8 2}$

Thermal correction to Enthalpy $=\mathbf{0 . 0 4 0 7 7 0}$

Acetylene:

Charge $=0$ Multiplicity $=1$

$\begin{array}{cccc}\mathrm{C} & -0.602508 & 0.000000 & 0.000000 \\ \mathrm{C} & 0.602538 & 0.000000 & 0.000000 \\ \mathrm{H} & 1.669255 & 0.000000 & 0.000000 \\ \mathrm{H} & -1.669434 & 0.000000 & 0.000000 \\ \mathrm{XX} & 0.602538 & 1.000000 & 0.000000 \\ \mathrm{XX} & -0.602508 & 1.000000 & 0.000000\end{array}$

$\mathrm{HF}=\mathbf{- 7 7 . 3 2 5 6 4 6 1}$

Thermal correction to Enthalpy= 0.030506

Acetylene $\mathrm{C} 1$ radical:

Charge $=0$ Multiplicity $=2$

$\begin{array}{cccc}\mathrm{C} & -0.733242 & 0.000000 & 0.000000 \\ \mathrm{C} & 0.475983 & 0.000000 & 0.000000 \\ \mathrm{H} & 1.543553 & 0.000000 & 0.000000 \\ \mathrm{XX} & 0.475983 & 1.000000 & 0.000000\end{array}$

$\mathrm{HF}=\mathbf{- 7 6 . 6 0 4 2 8 1 5}$

Thermal correction to Enthalpy $=\mathbf{0 . 0 1 8 3 2 2}$

\section{Cyclopropane:}

\section{Charge $=0$ Multiplicity $=1$}

$\begin{array}{lrrr}\text { C } & -0.435587 & -0.000012 & -0.754515 \\ \mathrm{C} & -0.435721 & 0.000034 & 0.754494 \\ \mathrm{C} & 0.871266 & -0.000036 & 0.000050 \\ \mathrm{H} & -0.731062 & -0.911469 & -1.267024 \\ \mathrm{H} & 1.463362 & 0.911104 & -0.000109 \\ \mathrm{H} & 1.463029 & -0.911398 & -0.000027 \\ \mathrm{H} & -0.732039 & -0.910987 & 1.267290 \\ \mathrm{H} & -0.731293 & 0.911622 & 1.266749\end{array}$


$\begin{array}{llll}\mathrm{H} & -0.731742 & 0.911205 & -1.267050\end{array}$

$\mathrm{HF}=\mathbf{- 1 1 7 . 8 9 5 2 0 2 4}$

Thermal correction to Enthalpy $=\mathbf{0 . 0 8 6 0 4 3}$

Cyclopropane $\mathrm{C} 1$ radical:

Charge $=0$ Multiplicity $=2$

$\begin{array}{lrrr}\text { C } & -0.465307 & 0.029454 & -0.757950 \\ \text { C } & -0.464429 & 0.027597 & 0.711918 \\ \text { C } & 0.845024 & 0.027598 & -0.091958 \\ \text { H } & -0.906432 & -0.650883 & -1.476507 \\ \text { H } & 1.431710 & 0.946597 & -0.072437 \\ \text { H } & 1.456711 & -0.875101 & -0.114304 \\ \text { H } & -0.761182 & -0.875102 & 1.247265 \\ \text { H } & -0.712533 & 0.946597 & 1.243920 \\ & & & \\ \text { HF = - } \mathbf{1 1 7 . 2 1 3 4 5 5 8} & & \end{array}$

Thermal correction to Enthalpy $=\mathbf{0 . 0 7 1 5 3 6}$

\section{Cyclobutane:}

Charge $=0$ Multiplicity $=1$

$\begin{array}{lrrr}\text { C } & -0.758113 & -0.118882 & -0.776332 \\ \text { C } & -0.748518 & -0.168835 & 0.776386 \\ \text { C } & 0.803513 & -0.118861 & 0.729245 \\ \text { C } & 0.703119 & 0.406578 & -0.729300 \\ \text { H } & -0.812990 & -1.122308 & -1.212820 \\ \text { H } & -1.169373 & 0.743764 & 1.212783 \\ \text { H } & -1.216679 & -1.031491 & 1.261894 \\ \text { H } & 1.316160 & 0.510584 & 1.464133 \\ \text { H } & 1.241624 & -1.122323 & 0.768260 \\ \text { H } & 0.740739 & 1.500867 & -0.768223 \\ \text { H } & 1.411681 & 0.010325 & -1.464141 \\ \text { H } & -1.511161 & 0.510581 & -1.261886 \\ & & & \\ \text { HF = -157.2131997 } & & \end{array}$

Thermal correction to Enthalpy $=\mathbf{0 . 1 1 6 5 2 8}$

Cyclobutane $\mathrm{C} 1$ radical:

Charge $=0$ Multiplicity $=2$
C $\quad-0.798966$
$-0.069832 \quad-0.724401$
$\begin{array}{llll}\text { C } & -0.788250 & 0.139037 & 0.763453\end{array}$
$\begin{array}{llll}\text { C } & 0.773516 & 0.067904 & 0.701274\end{array}$
$\begin{array}{llll}\text { C } & 0.696481 & -0.148677 & -0.846369\end{array}$
$\mathrm{H} \quad-1.601444 \quad-0.128268 \quad-1.454079$ 


$\begin{array}{cccc}\mathrm{H} & -1.196675 & 1.097997 & 1.119387 \\ \mathrm{H} & -1.265838 & -0.650571 & 1.364787 \\ \mathrm{H} & 1.280408 & 0.988652 & 1.004218 \\ \mathrm{H} & 1.211625 & -0.767740 & 1.254689 \\ \mathrm{H} & 1.173828 & 0.638637 & -1.450836 \\ \mathrm{H} & 1.101410 & -1.109301 & -1.201907\end{array}$

$\mathrm{HF}=-\mathbf{1 5 6 . 5 4 5 3 2 6 4}$

Thermal correction to Enthalpy= $\mathbf{0 . 1 0 1 3 7 3}$

\section{Cyclopentane:}

\section{Charge $=\mathbf{0}$ Multiplicity $=1$}

$\begin{array}{rrrr}\mathrm{C} & -0.913441 & -0.405864 & -0.838888 \\ \mathrm{C} & -0.961790 & -0.490823 & 0.706649 \\ \mathrm{C} & 0.502147 & -0.309496 & 1.140551 \\ \mathrm{C} & 1.014214 & 0.777786 & 0.181273 \\ \mathrm{C} & 0.361029 & 0.427612 & -1.179211 \\ \mathrm{H} & 0.610368 & -0.039047 & 2.197194 \\ \mathrm{H} & 0.664802 & 1.760173 & 0.525694 \\ \mathrm{H} & 2.107374 & 0.826816 & 0.127095 \\ \mathrm{H} & 0.125534 & 1.327082 & -1.758136 \\ \mathrm{H} & 1.050645 & -0.165200 & -1.791368 \\ \mathrm{H} & -1.824014 & 0.041604 & -1.251488 \\ \mathrm{H} & -0.833606 & -1.410032 & -1.271632 \\ \mathrm{H} & -1.565973 & 0.331814 & 1.111705 \\ \mathrm{H} & -1.407109 & -1.424939 & 1.066483 \\ \mathrm{H} & 1.059028 & -1.243559 & 0.982215 \\ & & & \\ \mathbf{H F}=\mathbf{- 1 9 6 . 5 5 7 0 7 5} & & \end{array}$

Thermal correction to Enthalpy $=\mathbf{0 . 1 4 7 5 6 0}$

Cyclopentane $\mathrm{C} 1$ radical:

\section{Charge $=0$ Multiplicity $=2$}

$\begin{array}{lrrr}\mathrm{C} & -0.149017 & -0.541100 & -1.121439 \\ \mathrm{C} & -0.613252 & 0.859740 & -0.676274 \\ \mathrm{C} & -0.590100 & 0.791700 & 0.870089 \\ \mathrm{C} & 0.491670 & -0.206148 & 1.160476 \\ \mathrm{C} & 0.925995 & -0.931820 & -0.078429 \\ \mathrm{H} & -0.416078 & 1.772359 & 1.336283 \\ \mathrm{H} & 1.925668 & -0.596672 & -0.411558 \\ \mathrm{H} & 0.223173 & -0.561505 & -2.151382 \\ \mathrm{H} & 0.106357 & 1.614214 & -1.019720 \\ \mathrm{H} & -1.593573 & 1.134362 & -1.080344 \\ \mathrm{H} & -1.567864 & 0.448609 & 1.255778\end{array}$




$\begin{array}{rrrr}\text { H } & 0.909951 & -0.380798 & 2.146721 \\ \text { H } & 1.009636 & -2.019528 & 0.059645 \\ \text { H } & -0.989047 & -1.245273 & -1.061962 \\ & & & \\ \text { HF=-195.8948572 } & & \end{array}$

Thermal correction to Enthalpy= 0.132609

\section{Cyclohexane:}

\section{Charge $=0$ Multiplicity $=1$}

$\begin{array}{lrrr}\text { C } & -1.125475 & -0.589527 & -0.768346 \\ \mathrm{C} & -1.120695 & -0.598859 & 0.768679 \\ \mathrm{C} & 0.311784 & -0.589357 & 1.326286 \\ \mathrm{C} & 1.125475 & 0.589528 & 0.768346 \\ \mathrm{C} & 1.120695 & 0.598859 & -0.768678 \\ \mathrm{C} & -0.311783 & 0.589356 & -1.326286 \\ \mathrm{H} & 0.292852 & -0.553138 & 2.423168 \\ \mathrm{H} & 0.691985 & 1.532133 & 1.135297 \\ \mathrm{H} & 2.155602 & 0.553153 & 1.145635 \\ \mathrm{H} & 1.669740 & 1.471487 & -1.145427 \\ \mathrm{H} & 1.656725 & -0.289155 & -1.136211 \\ \mathrm{H} & -0.810242 & 1.531875 & -1.053890 \\ \mathrm{H} & -0.292851 & 0.553135 & -2.423169 \\ \mathrm{H} & -2.155603 & -0.553151 & -1.145634 \\ \mathrm{H} & -0.691988 & -1.532133 & -1.135297 \\ \mathrm{H} & -1.656725 & 0.289155 & 1.136211 \\ \mathrm{H} & -1.669739 & -1.471487 & 1.145428 \\ \mathrm{H} & 0.810242 & -1.531875 & 1.053887 \\ & & & \\ \text { HF= } & \mathbf{- 2 3 5 . 8 8 0 4 8 7 2} & & \end{array}$

Thermal correction to Enthalpy $=\mathbf{0 . 1 7 7 8 4 1}$

Cyclohexane $\mathrm{C} 1$ radical:

\section{Charge $=0$ Multiplicity $=2$}

$\begin{array}{lrrr}\mathrm{C} & -1.131470 & -0.573431 & -0.752856 \\ \mathrm{C} & -1.082113 & -0.715201 & 0.783866 \\ \mathrm{C} & 0.312696 & -0.590882 & 1.313440 \\ \mathrm{C} & 1.199947 & 0.492518 & 0.783887 \\ \mathrm{C} & 1.110505 & 0.613074 & -0.752835 \\ \mathrm{C} & -0.349690 & 0.660784 & -1.227329 \\ \mathrm{H} & 0.903574 & 1.467788 & 1.220157 \\ \mathrm{H} & 2.239972 & 0.332974 & 1.095823 \\ \mathrm{H} & 1.653464 & 1.504959 & -1.090634 \\ \mathrm{H} & 1.607377 & -0.254141 & -1.209745 \\ \mathrm{H} & -0.830865 & 1.569986 & -0.835506\end{array}$




$\begin{array}{cccc}\text { H } & -0.388743 & 0.734595 & -2.321827 \\ \text { H } & -2.174294 & -0.520779 & -1.090670 \\ \text { H } & -0.693841 & -1.471999 & -1.209766 \\ \text { H } & -1.721850 & 0.078353 & 1.220132 \\ \text { H } & -1.535113 & -1.664888 & 1.095788 \\ \text { H } & 0.581073 & -1.098013 & 2.237206 \\ \mathbf{H F}=\mathbf{- 2 3 5 . 2 1 3 9 1 6 3} & & \end{array}$

Thermal correction to Enthalpy $=\mathbf{0 . 1 6 3 3 2 0}$

Cycloheptane:

Charge $=0$ Multiplicity $=1$

$\begin{array}{rrrr}\mathrm{C} & -1.081479 & 0.933907 & -0.746365 \\ \mathrm{C} & -1.082049 & 0.933170 & 0.794050 \\ \mathrm{C} & 0.299131 & 0.933223 & 1.481899 \\ \mathrm{C} & 1.408787 & 0.086138 & 0.824682 \\ \mathrm{C} & 0.975688 & -1.263879 & 0.222399 \\ \mathrm{C} & 0.423639 & -1.152501 & -1.210144 \\ \mathrm{C} & -0.943353 & -0.468913 & -1.364702 \\ \mathrm{H} & -0.294732 & 1.599719 & -1.130686 \\ \mathrm{H} & -1.718480 & -1.112767 & -0.922205 \\ \mathrm{H} & 1.165249 & -0.613263 & -1.818754 \\ \mathrm{H} & 0.243327 & -1.759889 & 0.876308 \\ \mathrm{H} & -1.657916 & 0.059514 & 1.130146 \\ \mathrm{H} & 0.157190 & 0.597531 & 2.518228 \\ \mathrm{H} & -2.031780 & 1.360241 & -1.094199 \\ \mathrm{H} & -1.176180 & -0.411211 & -2.437023 \\ \mathrm{H} & 0.352309 & -2.159169 & -1.644819 \\ \mathrm{H} & -1.637366 & 1.807112 & 1.159224 \\ \mathrm{H} & 1.849801 & -1.927866 & 0.192897 \\ \mathrm{H} & 0.664722 & 1.966682 & 1.553479 \\ \mathrm{H} & 2.189726 & -0.083823 & 1.577511 \\ \mathrm{H} & 1.891945 & 0.670319 & 0.028976 \\ & & & \\ \text { HF }= & \mathbf{- 2 7 5 . 1 8 3 5 4 9} & & \\ \mathrm{T} & & & \end{array}$

Thermal correction to Enthalpy= $\mathbf{0 . 2 0 8 1 1 4}$

Cycloheptane $\mathrm{C} 1$ radical:

Charge $=\mathbf{0}$ Multiplicity $=2$

$\begin{array}{lrrr}\mathrm{C} & -1.171470 & 0.882383 & -0.679479 \\ \mathrm{C} & -1.021547 & 0.912877 & 0.853504 \\ \mathrm{C} & 0.423272 & 1.041928 & 1.363148 \\ \mathrm{C} & 1.379734 & -0.034523 & 0.949793 \\ \mathrm{C} & 1.006408 & -1.279845 & 0.208007 \\ \mathrm{C} & 0.498391 & -1.045659 & -1.239194\end{array}$




$\begin{array}{lrrr}\mathrm{C} & -0.927684 & -0.486813 & -1.335847 \\ \mathrm{H} & -0.501561 & 1.632740 & -1.125714 \\ \mathrm{H} & -1.622403 & -1.215350 & -0.890298 \\ \mathrm{H} & 1.198011 & -0.375855 & -1.758032 \\ \mathrm{H} & 0.226180 & -1.843950 & 0.749146 \\ \mathrm{H} & -1.491491 & 0.015565 & 1.281036 \\ \mathrm{H} & 0.397393 & 1.111864 & 2.466438 \\ \mathrm{H} & -2.191819 & 1.197631 & -0.936258 \\ \mathrm{H} & -1.202079 & -0.418564 & -2.397714 \\ \mathrm{H} & 0.528597 & -2.004144 & -1.776151 \\ \mathrm{H} & -1.589225 & 1.765110 & 1.249389 \\ \mathrm{H} & 1.880573 & -1.940955 & 0.166965 \\ \mathrm{H} & 0.819504 & 2.020882 & 1.039919 \\ \mathrm{H} & 2.425701 & 0.112933 & 1.211679 \\ & & & \\ \text { HF= -274.5225709 } & & \end{array}$

Thermal correction to Enthalpy= $\mathbf{0 . 1 9 3 3 3 5}$

Spiro[2.2]pentane:

Charge $=0$ Multiplicity $=1$

$\begin{array}{lrrr}\text { C } & -1.368759 & -0.123078 & -0.569121 \\ \mathrm{C} & -1.097110 & -0.416926 & 0.906446 \\ \mathrm{C} & -0.000127 & -0.002628 & -0.005617 \\ \mathrm{C} & 1.093319 & 1.002789 & 0.004169 \\ \mathrm{C} & 1.372747 & -0.460981 & -0.338259 \\ \mathrm{H} & -1.913536 & 0.786617 & -0.813952 \\ \mathrm{H} & -1.593654 & -0.965846 & -1.219720 \\ \mathrm{H} & -1.142201 & -1.454918 & 1.230368 \\ \mathrm{H} & -1.462075 & 0.296299 & 1.642680 \\ \mathrm{H} & 1.449942 & 1.378550 & 0.961090 \\ \mathrm{H} & 1.141947 & 1.727831 & -0.805914 \\ \mathrm{H} & 1.607398 & -0.703089 & -1.372913 \\ \mathrm{H} & 1.911765 & -1.060502 & 0.392653\end{array}$

$\mathrm{HF}=\mathbf{- 1 9 5 . 2 7 1 5 3 1 6}$

Thermal correction to Enthalpy= 0.121404

\section{Spiro[2.2]pentane $\mathrm{C1}$ radical:}

Charge $=0$ Multiplicity $=2$

$\begin{array}{lrrr}\text { C } & 0.007915 & -0.041347 & -1.450853 \\ \text { C } & 0.048751 & -0.020791 & 0.044020 \\ \text { C } & 1.019508 & 0.041115 & 1.101752 \\ \text { C } & -0.130901 & 0.986380 & 1.155691 \\ \text { C } & -0.809965 & -1.037122 & -0.645347\end{array}$




$\begin{array}{cccc}\text { H } & -0.525273 & 0.760498 & -1.958123 \\ \text { H } & 0.882159 & -0.407126 & -1.984982 \\ \text { H } & -0.484033 & -2.074708 & -0.628712 \\ \text { H } & -1.888550 & -0.901387 & -0.611254 \\ \text { H } & -0.925248 & 0.804217 & 1.882416 \\ \text { H } & 0.037408 & 2.046182 & 0.951379 \\ \text { H } & 2.091684 & 0.202909 & 1.117700 \\ & & & \\ \text { HF }=\mathbf{- 1 9 4 . 5 9 2 8 3 4 7} & & \end{array}$

Thermal correction to Enthalpy=0.107176

Bicyclo[1.1.0]butane:

Charge $=0$ Multiplicity $=1$

$\begin{array}{lrcc}\mathrm{C} & -0.598110 & -0.230556 & -0.990544 \\ \mathrm{C} & -0.571627 & -0.270340 & 0.507572 \\ \mathrm{C} & 0.912828 & -0.230501 & 0.711113 \\ \mathrm{C} & 0.253291 & 0.736693 & -0.224928 \\ \mathrm{H} & -0.122640 & -1.034777 & -1.560726 \\ \mathrm{H} & -1.498634 & 0.167195 & -1.457860 \\ \mathrm{H} & 1.535284 & -1.034718 & 0.306469 \\ \mathrm{H} & 1.270317 & 0.167297 & 1.660582 \\ \mathrm{H} & -1.371532 & -0.113049 & 1.217801 \\ \mathrm{H} & 0.208906 & 1.816273 & -0.185542\end{array}$

$H F=\mathbf{- 1 5 5 . 9 4 8 0 4 8 4}$

Thermal correction to Enthalpy $=\mathbf{0 . 0 9 1 4 8 3}$

Bicyclo[1.1.0]butane $\mathrm{C} 1$ radical:

Charge $=0$ Multiplicity $=2$

$\begin{array}{lrrr}\text { C } & -0.658057 & 0.244322 & -0.967595 \\ \mathrm{C} & -0.607006 & 0.251681 & 0.520516 \\ \mathrm{C} & 0.855908 & 0.244324 & 0.797934 \\ \mathrm{C} & 0.209391 & -0.726910 & -0.179555 \\ \mathrm{H} & -0.199530 & 1.049588 & -1.551892 \\ \mathrm{H} & -1.548484 & -0.186232 & -1.423429 \\ \mathrm{H} & 1.503303 & 1.049590 & 0.433888 \\ \mathrm{H} & 1.170604 & -0.186230 & 1.747467 \\ \mathrm{H} & 0.272696 & -1.807216 & -0.233839 \\ & & & \\ \text { HF= -155.2605708 } & & \end{array}$

Thermal correction to Enthalpy= $\mathbf{0 . 0 7 8 4 0 7}$

Bicyclo[1.1.0]butane $\mathrm{C} 2$ radical:

Charge $=0$ Multiplicity $=2$ 


$\begin{array}{lrrr}\text { C } & -0.536607 & 0.271168 & -0.975967 \\ \text { C } & -0.533825 & 0.328889 & 0.523324 \\ \text { C } & 0.902967 & 0.221287 & 0.767230 \\ \text { C } & 0.375650 & -0.678283 & -0.256562 \\ \text { H } & -0.088870 & 1.100923 & -1.525407 \\ \text { H } & -1.418641 & -0.154647 & -1.454653 \\ \text { H } & 1.338816 & -0.111901 & 1.705792 \\ \text { H } & 0.284090 & -1.759218 & -0.243789 \\ \text { H } & -1.364511 & 0.066476 & 1.169906 \\ & & & \\ \text { HF= -155.2789111 } & & \end{array}$

Thermal correction to Enthalpy $=\mathbf{0 . 0 7 8 1 9 1}$

\section{Bicyclo[2.1.0]pentane:}

\section{Charge $=0$ Multiplicity $=1$}

$\begin{array}{cccc}\mathrm{C} & -0.727449 & 0.522428 & -1.091899 \\ \mathrm{C} & -0.697458 & 0.529226 & 0.411899 \\ \mathrm{C} & 0.751363 & 0.512357 & 0.915660 \\ \mathrm{C} & 0.994319 & -0.814048 & 0.119474 \\ \mathrm{C} & -0.459664 & -0.768155 & -0.367267 \\ \mathrm{H} & 0.830238 & 0.363196 & 1.996661 \\ \mathrm{H} & 1.365760 & 1.378349 & 0.638520 \\ \mathrm{H} & 1.763287 & -0.790649 & -0.663057 \\ \mathrm{H} & 1.200348 & -1.659519 & 0.782641 \\ \mathrm{H} & -1.113977 & -1.628881 & -0.476707 \\ \mathrm{H} & -1.563764 & 0.825389 & 0.997345 \\ \mathrm{H} & -1.713894 & 0.611290 & -1.540892 \\ \mathrm{H} & 0.065337 & 1.009970 & -1.661710\end{array}$

$H F=\mathbf{- 1 9 5 . 2 8 0 2 3 3 9}$

Thermal correction to Enthalpy $=\mathbf{0 . 1 2 2 1 8 6}$

Bicyclo[2.1.0]pentane $\mathrm{C} 1$ radical:

Charge $=0$ Multiplicity $=2$

$\begin{array}{lrrr}\mathrm{C} & -0.671331 & -0.587616 & -0.390312 \\ \mathrm{C} & -0.705009 & -0.637293 & 1.084917 \\ \mathrm{C} & 0.643828 & -0.638967 & 0.346456 \\ \mathrm{C} & 0.965584 & 0.764775 & -0.178850 \\ \mathrm{C} & -0.392469 & 0.786935 & -0.976440 \\ \mathrm{H} & 1.843382 & 0.799462 & -0.829975 \\ \mathrm{H} & 1.058608 & 1.539882 & 0.593248 \\ \mathrm{H} & -1.111283 & 1.577254 & -0.729700 \\ \mathrm{H} & -0.225560 & 0.781341 & -2.057350 \\ \mathrm{H} & 1.358632 & -1.443350 & 0.496748\end{array}$




$$
\begin{array}{crrr}
\text { H } & -0.951613 & -1.601888 & 1.525113 \\
\text { H } & -1.015783 & 0.220298 & 1.687293 \\
& & & \\
\text { HF }= & \mathbf{- 1 9 4 . 5 9 8 3 3 5 9} & &
\end{array}
$$

Thermal correction to Enthalpy= 0.109012

\section{Bicyclo[2.1.0]pentane $\mathrm{C} 2$ radical:}

Charge $=0$ Multiplicity $=2$

$\begin{array}{lrrr}\text { C } & -0.660780 & -0.568875 & -0.445675 \\ \text { C } & -0.722551 & -0.509684 & 1.078692 \\ \text { C } & 0.622028 & -0.524263 & 0.430439 \\ \text { C } & 0.974268 & 0.855119 & -0.166796 \\ \text { C } & -0.299858 & 0.757313 & -0.990357 \\ \text { H } & 1.882232 & 0.827162 & -0.785493 \\ \text { H } & 1.076221 & 1.683526 & 0.549065 \\ \text { H } & -0.554744 & 1.216228 & -1.938542 \\ \text { H } & -1.100357 & -1.406395 & -0.981104 \\ \text { H } & 1.334182 & -1.327924 & 0.592230 \\ \text { H } & -1.021494 & -1.439842 & 1.557971 \\ \text { H } & -1.094681 & 0.389580 & 1.568052 \\ & & & \\ \text { HF = -194.6122336 } & & \end{array}$

Thermal correction to Enthalpy $=\mathbf{0 . 1 0 7 6 1 7}$

Bicyclo[2.1.0]pentane $\mathrm{C5}$ radical:

Charge $=0$ Multiplicity $=2$

$\begin{array}{lrrr}\text { C } & -0.713805 & -0.571714 & -0.367701 \\ \mathrm{C} & -0.643510 & -0.677001 & 1.087869 \\ \mathrm{C} & 0.666130 & -0.571193 & 0.448897 \\ \mathrm{C} & 0.946787 & 0.848235 & -0.071028 \\ \mathrm{C} & -0.393739 & 0.847650 & -0.864567 \\ \mathrm{H} & 1.815420 & 0.887559 & -0.734380 \\ \mathrm{H} & 1.062261 & 1.612340 & 0.705699 \\ \mathrm{H} & -1.130664 & 1.611467 & -0.592522 \\ \mathrm{H} & -0.230042 & 0.886580 & -1.945217 \\ \mathrm{H} & -1.141961 & -1.350834 & -0.995681 \\ \mathrm{H} & 1.423121 & -1.349842 & 0.522217 \\ \mathrm{H} & -0.969315 & -1.553133 & 1.639065 \\ & & & \\ \text { HF }=\mathbf{- 1 9 4 . 6 0 3 7 6 8 5} & & \end{array}$

Thermal correction to Enthalpy= 0.108609

\section{Bicyclo[3.1.0]hexane:}




\begin{tabular}{cccc}
\multicolumn{5}{c}{ Charge $=\mathbf{0}$ Multiplicity $\mathbf{1}$} \\
$\mathrm{C}$ & -0.743984 & 0.833110 & -1.273223 \\
$\mathrm{C}$ & -0.721211 & 0.833388 & 0.235513 \\
$\mathrm{C}$ & 0.649593 & 0.822494 & 0.922151 \\
$\mathrm{C}$ & 1.093719 & -0.661960 & 0.908329 \\
$\mathrm{C}$ & 0.394589 & -1.300737 & -0.318140 \\
$\mathrm{C}$ & -0.877904 & -0.471284 & -0.526614 \\
$\mathrm{H}$ & 0.728345 & -1.150749 & 1.820198 \\
$\mathrm{H}$ & 2.183177 & -0.783349 & 0.892139 \\
$\mathrm{H}$ & 1.366510 & 1.454220 & 0.380835 \\
$\mathrm{H}$ & 0.591119 & 1.216752 & 1.942592 \\
$\mathrm{H}$ & -1.564253 & 1.275920 & 0.760088 \\
$\mathrm{H}$ & -1.835712 & -0.984321 & -0.560237 \\
$\mathrm{H}$ & 0.161884 & -2.357180 & -0.145129 \\
$\mathrm{H}$ & 1.040346 & -1.261517 & -1.205570 \\
$\mathrm{H}$ & 0.186948 & 1.031101 & -1.803560 \\
$\mathrm{H}$ & -1.627177 & 1.229051 & -1.769446 \\
& & & \\
$\mathbf{H F}=\mathbf{- 2 3 4 . 6 2 5 7 1 8 5}$ & & \\
\hline
\end{tabular}

Thermal correction to Enthalpy= $\mathbf{0 . 1 5 3 1 2 1}$

Bicyclo[3.1.0]hexane $\mathrm{C} 1$ radical:

Charge $=\mathbf{0}$ Multiplicity $=2$

$\begin{array}{lrrr}\text { C } & -0.812694 & -0.066268 & -0.758175 \\ \mathrm{C} & -0.857540 & -0.143568 & 0.723389 \\ \mathrm{C} & 0.597856 & -0.178496 & 1.208846 \\ \mathrm{C} & 1.413335 & -0.638395 & -0.029742 \\ \mathrm{C} & 0.604203 & -0.163985 & -1.273153 \\ \mathrm{C} & -1.346037 & 1.106651 & -0.042383 \\ \mathrm{H} & 1.464507 & -1.733710 & -0.037004 \\ \mathrm{H} & 2.443295 & -0.264187 & -0.027144 \\ \mathrm{H} & 0.917509 & 0.819182 & 1.539920 \\ \mathrm{H} & 0.731090 & -0.853964 & 2.060424 \\ \mathrm{H} & -1.617652 & -0.698265 & 1.270904 \\ \mathrm{H} & 0.694865 & -0.871112 & -2.106559 \\ \mathrm{H} & 0.956381 & 0.808444 & -1.646231 \\ \mathrm{H} & -0.761064 & 2.028785 & -0.005345 \\ \mathrm{H} & -2.423662 & 1.269197 & -0.021654 \\ & & & \\ \text { HF }= & \mathbf{- 2 3 3 . 9 4 6 6 9 1 5} & & \end{array}$

Thermal correction to Enthalpy=0.139582

Bicyclo[3.1.0]hexane $\mathrm{C} 2$ radical:

Charge $=0$ Multiplicity $=2$ 


$\begin{array}{rrrr}\text { C } & -0.872345 & -0.197062 & -0.747559 \\ \text { C } & -0.816259 & 0.055228 & 0.757039 \\ \text { C } & 0.603662 & -0.253156 & 1.228246 \\ \text { C } & 1.467923 & -0.333925 & -0.064617 \\ \text { C } & 0.476762 & -0.596882 & -1.162942 \\ \text { C } & -1.022597 & 1.220629 & -0.163436 \\ \text { H } & 2.243667 & -1.108549 & 0.005578 \\ \text { H } & 2.013194 & 0.612558 & -0.227043 \\ \text { H } & 0.986906 & 0.488674 & 1.939216 \\ \text { H } & 0.604431 & -1.223510 & 1.739575 \\ \text { H } & -1.645063 & -0.246016 & 1.392885 \\ \text { H } & -1.747963 & -0.629608 & -1.225416 \\ \text { H } & 0.752732 & -0.852665 & -2.180615 \\ \text { H } & -0.214047 & 1.930412 & -0.322822 \\ \text { H } & -2.016734 & 1.659706 & -0.201735 \\ & & & \\ \text { HF }=\mathbf{- 2 3 3 . 9 6 7 8 1 5 7} & & \end{array}$

Thermal correction to Enthalpy $=\mathbf{0 . 1 3 8 6 6 0}$

\section{Bicyclo[3.1.0]hexane $\mathrm{C} 3$ radical:}

\section{Charge $=0$ Multiplicity $=2$}

$\begin{array}{lrrr}\mathrm{C} & -0.815852 & -0.100007 & -0.760029 \\ \mathrm{C} & -0.815849 & -0.100008 & 0.760033 \\ \mathrm{C} & 0.607952 & -0.365345 & 1.245854 \\ \mathrm{C} & 1.441385 & -0.276852 & -0.000004 \\ \mathrm{C} & 0.607946 & -0.365344 & -1.245857 \\ \mathrm{C} & -1.047042 & 1.183220 & 0.000003 \\ \mathrm{H} & 2.526747 & -0.276209 & -0.000006 \\ \mathrm{H} & 0.934163 & 0.342043 & 2.024276 \\ \mathrm{H} & 0.668347 & -1.368127 & 1.707522 \\ \mathrm{H} & -1.645105 & -0.526906 & 1.317872 \\ \mathrm{H} & -1.645112 & -0.526905 & -1.317864 \\ \mathrm{H} & 0.668338 & -1.368127 & -1.707526 \\ \mathrm{H} & 0.934153 & 0.342044 & -2.024281 \\ \mathrm{H} & -0.259073 & 1.934914 & 0.000001 \\ \mathrm{H} & -2.053694 & 1.593287 & 0.000005 \\ & & & \\ \text { HF }=\mathbf{- 2 3 3 . 9 6 6 7 6 2 6} & & \end{array}$

Thermal correction to Enthalpy= $\mathbf{0 . 1 3 8 3 5 3}$

Bicyclo[3.1.0]hexane C6 radical:

Charge $=0$ Multiplicity $=2$ 


$\begin{array}{rrrr}\text { C } & -0.843165 & -0.078018 & -0.776144 \\ \mathrm{C} & -0.843161 & -0.078017 & 0.776148 \\ \mathrm{C} & 0.629392 & -0.078104 & 1.233427 \\ \mathrm{C} & 1.434648 & -0.546782 & -0.000003 \\ \mathrm{C} & 0.629386 & -0.078105 & -1.233430 \\ \mathrm{C} & -1.333556 & 1.069114 & 0.000002 \\ \mathrm{H} & 1.478848 & -1.643412 & -0.000002 \\ \mathrm{H} & 2.467979 & -0.180209 & -0.000005 \\ \mathrm{H} & 0.936344 & 0.926782 & 1.547232 \\ \mathrm{H} & 0.774371 & -0.742745 & 2.092140 \\ \mathrm{H} & -1.551312 & -0.676692 & 1.349732 \\ \mathrm{H} & -1.551318 & -0.676694 & -1.349724 \\ \mathrm{H} & 0.774361 & -0.742747 & -2.092142 \\ \mathrm{H} & 0.936337 & 0.926780 & -1.547237 \\ \mathrm{H} & -2.306877 & 1.548411 & 0.000004 \\ & & & \\ \text { HF }=\mathbf{- 2 3 3 . 9 4 5 3 7 4 6} & & \end{array}$

Thermal correction to Enthalpy=0.139139

\section{Bicyclo[2.2.0]hexane:}

\section{Charge $=0$ Multiplicity $=1$}

\begin{tabular}{lrrr}
$\mathrm{C}$ & -0.520212 & -0.781960 & -1.225680 \\
$\mathrm{C}$ & -0.486227 & -0.781979 & 0.326565 \\
$\mathrm{C}$ & 0.933570 & -0.781933 & 0.953626 \\
$\mathrm{C}$ & 0.946951 & 0.777829 & 0.939488 \\
$\mathrm{C}$ & -0.476163 & 0.791298 & 0.318857 \\
$\mathrm{C}$ & -0.517528 & 0.777904 & -1.232641 \\
$\mathrm{H}$ & -1.434247 & -1.214782 & -1.645310 \\
$\mathrm{H}$ & 0.335773 & -1.267599 & -1.708941 \\
$\mathrm{H}$ & 0.336055 & 1.257726 & -1.725921 \\
$\mathrm{H}$ & -1.433689 & 1.208497 & -1.650022 \\
$\mathrm{H}$ & 1.707399 & -1.277518 & 0.355508 \\
$\mathrm{H}$ & 0.966304 & -1.203968 & 1.963469 \\
$\mathrm{H}$ & 0.994125 & 1.219054 & 1.940477 \\
$\mathrm{H}$ & 1.725309 & 1.247634 & 0.326676 \\
$\mathrm{H}$ & -1.229050 & 1.400898 & 0.827191 \\
$\mathrm{H}$ & -1.250315 & -1.376892 & 0.835586 \\
& & & \\
HF= -234.5980467 & & \\
\hline
\end{tabular}

Thermal correction to Enthalpy= 0.152354

Bicyclo[2.2.0]hexane $\mathrm{C} 1$ radical:

Charge $=0$ Multiplicity $=2$

$\begin{array}{llll}\text { C } & -0.568681 & -0.823653 & -1.225892\end{array}$ 


$\begin{array}{rrrr}\text { C } & -0.427857 & -0.781361 & 0.288282 \\ \text { C } & 0.919184 & -0.820703 & 0.993924 \\ \text { C } & 0.907212 & 0.749634 & 0.984848 \\ \text { C } & -0.513128 & 0.758386 & 0.342996 \\ \text { C } & -0.569404 & 0.746704 & -1.214445 \\ \text { H } & -1.520420 & -1.241089 & -1.576812 \\ \text { H } & 0.242059 & -1.316822 & -1.777138 \\ \text { H } & 0.302019 & 1.202519 & -1.697512 \\ \text { H } & -1.475456 & 1.195789 & -1.633626 \\ \text { H } & 1.737654 & -1.315507 & 0.455752 \\ \text { H } & 0.881701 & -1.234167 & 2.009232 \\ \text { H } & 0.952655 & 1.202774 & 1.980306 \\ \text { H } & 1.683642 & 1.203216 & 0.358835 \\ \text { H } & -1.287815 & 1.329241 & 0.862680 \\ & & & \\ \text { HF }=\mathbf{- 2 3 3 . 9 2 5 7 7 1 6} & & \\ \text { Th }\end{array}$

Thermal correction to Enthalpy $=\mathbf{0 . 1 3 8 9 7 3}$

Bicyclo[2.2.0]hexane $\mathrm{C} 2$ radical:

Charge $=0$ Multiplicity $=2$

$\begin{array}{lrrr}\mathrm{C} & -0.639012 & -0.722320 & -1.175132 \\ \mathrm{C} & -0.535197 & -0.781975 & 0.318415 \\ \mathrm{C} & 0.923611 & -0.864353 & 0.890089 \\ \mathrm{C} & 1.037757 & 0.687275 & 0.818469 \\ \mathrm{C} & -0.425261 & 0.792787 & 0.306814 \\ \mathrm{C} & -0.583829 & 0.781202 & -1.244198 \\ \mathrm{H} & -0.569441 & -1.491300 & -1.940981 \\ \mathrm{H} & 0.240570 & 1.237291 & -1.814981 \\ \mathrm{H} & -1.515618 & 1.253407 & -1.596803 \\ \mathrm{H} & 1.635861 & -1.438838 & 0.289276 \\ \mathrm{H} & 0.950291 & -1.248243 & 1.915728 \\ \mathrm{H} & 1.200184 & 1.157788 & 1.793294 \\ \mathrm{H} & 1.792303 & 1.076886 & 0.125920 \\ \mathrm{H} & -1.104023 & 1.439303 & 0.869117 \\ \mathrm{H} & -1.298536 & -1.341982 & 0.872685 \\ & & & \\ \text { HF= -233.9318165 } & & \\ \text { T }\end{array}$

Thermal correction to Enthalpy= 0.137746

\section{Biocyclo[3.2.0]heptane:}

\section{Charge $=0$ Multiplicity $=1$}
C -0.914115
$0.506308 \quad-1.211845$
$\begin{array}{llll}\text { C } & -0.911802 & 0.488711 & 0.326591\end{array}$
$\begin{array}{llll}\text { C } & 0.601406 & 0.488740 & 0.759139\end{array}$ 


$\begin{array}{rrrr}\text { C } & 1.416471 & 0.506517 & -0.545627 \\ \mathrm{C} & 0.460973 & -0.066702 & -1.612470 \\ \mathrm{C} & 0.401136 & -0.905146 & 1.424669 \\ \mathrm{C} & -1.093535 & -0.905076 & 0.997611 \\ \mathrm{H} & -1.801840 & -0.912279 & 1.832410 \\ \mathrm{H} & -1.379024 & -1.707960 & 0.307681 \\ \mathrm{H} & 1.007956 & -1.707955 & 0.989468 \\ \mathrm{H} & 0.561564 & -0.912632 & 2.507647 \\ \mathrm{H} & 0.911780 & 1.274835 & 1.454791 \\ \mathrm{H} & -1.542963 & 1.274872 & 0.752966 \\ \mathrm{H} & 2.356713 & -0.053034 & -0.465523 \\ \mathrm{H} & 1.675599 & 1.542146 & -0.804891 \\ \mathrm{H} & 0.445550 & -1.163585 & -1.558174 \\ \mathrm{H} & 0.753263 & 0.197030 & -2.635058 \\ \mathrm{H} & -0.997254 & 1.541885 & -1.569001 \\ \mathrm{H} & -1.754546 & -0.053431 & -1.640723 \\ & & & \\ \text { HF }=\mathbf{- 2 7 3 . 9 4 8 0 2 6 7} & & \\ \text { Ther }\end{array}$

Thermal correction to Enthalpy $=\mathbf{0 . 1 8 3 3 5 3}$

Biocyclo[3.2.0]heptane $\mathrm{C} 1$ radical:

Charge $=0$ Multiplicity $=2$

$\begin{array}{rrrr}\text { C } & -1.473712 & 0.216689 & -0.864174 \\ \mathrm{C} & -1.457680 & 0.045489 & 0.683116 \\ \mathrm{C} & 0.010284 & 0.022413 & 1.014407 \\ \mathrm{C} & 0.812484 & 0.671819 & -0.116313 \\ \mathrm{C} & -0.228077 & 1.081409 & -1.178391 \\ \mathrm{C} & 0.899205 & -1.208228 & 1.060174 \\ \mathrm{C} & 1.554528 & -0.699992 & -0.265195 \\ \mathrm{H} & 1.605084 & -1.221095 & 1.901255 \\ \mathrm{H} & 0.395787 & -2.183507 & 1.028365 \\ \mathrm{H} & 1.220040 & -1.264286 & -1.142232 \\ \mathrm{H} & 2.649079 & -0.652464 & -0.276256 \\ \mathrm{H} & 1.491502 & 1.490308 & 0.151010 \\ \mathrm{H} & 0.129604 & 0.948845 & -2.206614 \\ \mathrm{H} & -0.471942 & 2.144896 & -1.055912 \\ \mathrm{H} & -1.375381 & -0.768788 & -1.337498 \\ \mathrm{H} & -2.403533 & 0.661307 & -1.236824 \\ \mathrm{H} & -1.944384 & 0.914220 & 1.153441 \\ \mathrm{H} & -1.998048 & -0.847032 & 1.019526 \\ & & & \\ \text { HF }=\mathbf{- 2 7 3 . 2 7 8 9 9 5 9} & & \end{array}$

Thermal correction to Enthalpy $=\mathbf{0 . 1 6 9 8 1 8}$ 
Biocyclo[3.2.0]heptane $\mathrm{C} 2$ radical:

Charge $=0$ Multiplicity $=2$

$\begin{array}{lrrr}\mathrm{C} & -1.502434 & 0.274636 & -0.901313 \\ \mathrm{C} & -1.408072 & 0.018699 & 0.574086 \\ \mathrm{C} & -0.013485 & 0.148271 & 1.087253 \\ \mathrm{C} & 0.807032 & 0.610634 & -0.166893 \\ \mathrm{C} & -0.204884 & 1.056913 & -1.232299 \\ \mathrm{C} & 0.840403 & -1.171885 & 1.123792 \\ \mathrm{C} & 1.396117 & -0.827495 & -0.283485 \\ \mathrm{H} & 1.611204 & -1.134477 & 1.901420 \\ \mathrm{H} & 0.286804 & -2.110750 & 1.227858 \\ \mathrm{H} & 0.926945 & -1.410598 & -1.084028 \\ \mathrm{H} & 2.481715 & -0.896953 & -0.406785 \\ \mathrm{H} & 1.574715 & 1.364044 & 0.034908 \\ \mathrm{H} & 0.051746 & 0.749816 & 2.004533 \\ \mathrm{H} & 0.149429 & 0.877422 & -2.254551 \\ \mathrm{H} & -0.390712 & 2.134138 & -1.135791 \\ \mathrm{H} & -1.541816 & -0.672109 & -1.471668 \\ \mathrm{H} & -2.410495 & 0.824073 & -1.186872 \\ \mathrm{H} & -2.227597 & -0.383238 & 1.164133 \\ & & & \\ \text { HF }=\mathbf{- 2 7 3 . 2 8 3 9 7 4 4} & & \end{array}$

Thermal correction to Enthalpy= 0.168915

Biocyclo[3.2.0]heptane $\mathrm{C} 3$ radical:

Charge $=0$ Multiplicity $=2$

\begin{tabular}{lrrr}
$\mathrm{C}$ & -1.498191 & 0.298597 & \multicolumn{1}{c}{-0.795795} \\
$\mathrm{C}$ & -1.471550 & 0.067103 & 0.685105 \\
$\mathrm{C}$ & 0.028225 & 0.064085 & 1.050445 \\
$\mathrm{C}$ & 0.803103 & 0.654551 & -0.186849 \\
$\mathrm{C}$ & -0.254026 & 0.994948 & -1.258949 \\
$\mathrm{C}$ & 0.775085 & -1.288495 & 0.859460 \\
$\mathrm{C}$ & 1.539662 & -0.705858 & -0.361547 \\
$\mathrm{H}$ & 1.414179 & -1.573183 & 1.701629 \\
$\mathrm{H}$ & 0.119520 & -2.136011 & 0.630230 \\
$\mathrm{H}$ & 1.336844 & -1.208254 & -1.313944 \\
$\mathrm{H}$ & 2.625518 & -0.650181 & -0.232888 \\
$\mathrm{H}$ & 1.468967 & 1.497711 & 0.020908 \\
$\mathrm{H}$ & 0.221119 & 0.546922 & 2.013314 \\
$\mathrm{H}$ & 0.059784 & 0.692992 & -2.268715 \\
$\mathrm{H}$ & -0.409202 & 2.089543 & -1.310105 \\
$\mathrm{H}$ & -2.396512 & 0.210618 & -1.400384 \\
$\mathrm{H}$ & -1.995094 & 0.880980 & 1.222078 \\
$\mathrm{H}$ & -1.978972 & -0.860727 & 0.986657
\end{tabular}


$\mathrm{HF}=\mathbf{- 2 7 3 . 2 8 2 0 9 4 8}$

Thermal correction to Enthalpy= $\mathbf{0 . 1 6 8 7 9 0}$

Biocyclo[3.2.0]heptane C6 radical:

Charge $=\mathbf{0}$ Multiplicity $=\mathbf{2}$

\begin{tabular}{lrrr} 
C & -1.362477 & 0.042003 & \multicolumn{1}{c}{-0.935821} \\
$\mathrm{C}$ & -1.432422 & 0.032372 & 0.605841 \\
$\mathrm{C}$ & 0.031933 & 0.086170 & 1.071673 \\
$\mathrm{C}$ & 0.847448 & 0.701974 & -0.134702 \\
$\mathrm{C}$ & -0.190421 & 0.993363 & -1.247126 \\
$\mathrm{C}$ & 0.852504 & -1.240032 & 0.930697 \\
$\mathrm{C}$ & 1.584926 & -0.605864 & -0.215478 \\
$\mathrm{H}$ & 1.468331 & -1.491989 & 1.808806 \\
$\mathrm{H}$ & 0.253767 & -2.135535 & 0.696041 \\
$\mathrm{H}$ & 2.344330 & -0.977517 & -0.899005 \\
$\mathrm{H}$ & 1.468585 & 1.583967 & 0.079215 \\
$\mathrm{H}$ & 0.152407 & 0.579511 & 2.040501 \\
$\mathrm{H}$ & 0.219188 & 0.855779 & -2.254434 \\
$\mathrm{H}$ & -0.524039 & 2.037219 & -1.167609 \\
$\mathrm{H}$ & -1.136289 & -0.964206 & -1.311365 \\
$\mathrm{H}$ & -2.303099 & 0.356228 & -1.401507 \\
$\mathrm{H}$ & -1.959348 & 0.931221 & 0.953689 \\
$\mathrm{H}$ & -1.972772 & -0.834594 & 1.005163 \\
& & & \\
HF= -273.2814598 & &
\end{tabular}

Thermal correction to Enthalpy= $\mathbf{0 . 1 6 8 6 0 7}$

Biocyclo[4.1.0]heptane:

Charge $=0$ Multiplicity $=1$

$\begin{array}{lrrr}\mathrm{C} & -1.013514 & 0.935558 & -0.721091 \\ \mathrm{C} & -1.014870 & 0.949670 & 0.816827 \\ \mathrm{C} & 0.419372 & 0.920653 & 1.359949 \\ \mathrm{C} & 1.141181 & -0.388330 & 0.993911 \\ \mathrm{C} & 0.955995 & -0.798292 & -0.471148 \\ \mathrm{C} & -0.132421 & -0.154599 & -1.314450 \\ \mathrm{C} & -0.262645 & -1.594173 & -0.887020 \\ \mathrm{H} & -1.542062 & 1.844165 & 1.171085 \\ \mathrm{H} & -1.574923 & 0.088037 & 1.208428 \\ \mathrm{H} & 0.422406 & 1.051356 & 2.449534 \\ \mathrm{H} & 0.971461 & 1.773950 & 0.939890 \\ \mathrm{H} & 0.780626 & -1.192780 & 1.650534 \\ \mathrm{H} & 2.211699 & -0.282683 & 1.207408 \\ \mathrm{H} & 1.876594 & -1.045690 & -0.995827\end{array}$




$\begin{array}{rrrr}\text { H } & 0.110715 & -0.001123 & -2.364998 \\ \text { H } & -0.640084 & 1.908900 & -1.071312 \\ \text { H } & -2.039416 & 0.841846 & -1.103767 \\ \text { H } & -0.986871 & -1.839301 & -0.112218 \\ \text { H } & -0.148738 & -2.369598 & -1.640624 \\ & & & \\ \text { HF }=\mathbf{- 2 7 3 . 9 4 5 9 3 5 1} & & \end{array}$

Thermal correction to Enthalpy= $\mathbf{0 . 1 8 3 7 9 8}$

Biocyclo[4.1.0]heptane $\mathrm{C} 1$ radical:

Charge $=0$ Multiplicity $=2$

$\begin{array}{lrrr}\text { C } & -1.190515 & -0.173388 & -0.595599 \\ \mathrm{C} & -1.209986 & -0.175487 & 0.931272 \\ \mathrm{C} & 0.204582 & -0.152420 & 1.540549 \\ \mathrm{C} & 1.062899 & 0.950932 & 0.902308 \\ \mathrm{C} & 1.318978 & 0.671086 & -0.599131 \\ \mathrm{C} & 0.079116 & 0.166555 & -1.272536 \\ \mathrm{C} & -0.400370 & -1.222469 & -1.400999 \\ \mathrm{H} & 0.130638 & 0.001236 & 2.624110 \\ \mathrm{H} & 0.696523 & -1.126130 & 1.401562 \\ \mathrm{H} & 2.021185 & 1.053161 & 1.426531 \\ \mathrm{H} & 0.539684 & 1.911339 & 1.008953 \\ \mathrm{H} & 2.129903 & -0.062775 & -0.713829 \\ \mathrm{H} & 1.674441 & 1.591748 & -1.084681 \\ \mathrm{H} & -2.130880 & 0.127360 & -1.061832 \\ \mathrm{H} & -1.746523 & 0.722401 & 1.269536 \\ \mathrm{H} & -1.778647 & -1.037503 & 1.307659 \\ \mathrm{H} & 0.134973 & -2.019057 & -0.879075 \\ \mathrm{H} & -0.859514 & -1.550631 & -2.334124 \\ & & & \\ \text { HF= } \mathbf{- 2 7 3 . 2 7 1 8 7 8 2} & & \end{array}$

Thermal correction to Enthalpy= 0.169877

Biocyclo[4.1.0]heptane $\mathrm{C} 2$ radical:

Charge $=\mathbf{0}$ Multiplicity $=2$

$\begin{array}{lrrr}\mathrm{C} & -1.161882 & -0.270850 & -0.572750 \\ \mathrm{C} & -1.159371 & -0.196861 & 0.947907 \\ \mathrm{C} & 0.253608 & -0.221764 & 1.554659 \\ \mathrm{C} & 1.158805 & 0.862160 & 0.941387 \\ \mathrm{C} & 1.159757 & 0.843260 & -0.557148 \\ \mathrm{C} & 0.040084 & 0.284604 & -1.323811 \\ \mathrm{C} & -0.270678 & -1.227878 & -1.308251 \\ \mathrm{H} & 0.187459 & -0.081381 & 2.640383 \\ \mathrm{H} & 0.707950 & -1.208939 & 1.399230\end{array}$




$\begin{array}{rrrr}\mathrm{H} & 2.182631 & 0.762536 & 1.329921 \\ \mathrm{H} & 0.809625 & 1.849396 & 1.303460 \\ \mathrm{H} & 1.935267 & 1.387771 & -1.089769 \\ \mathrm{H} & -0.156167 & 0.760865 & -2.283635 \\ \mathrm{H} & -2.133881 & -0.084532 & -1.027487 \\ \mathrm{H} & -1.657996 & 0.735456 & 1.250976 \\ \mathrm{H} & -1.758261 & -1.019167 & 1.363183 \\ \mathrm{H} & 0.398902 & -1.871436 & -0.745090 \\ \mathrm{H} & -0.637463 & -1.666587 & -2.233127 \\ & & & \\ \mathbf{H F}=\mathbf{- 2 7 3 . 2 8 7 1 0 0} & & \end{array}$

Thermal correction to Enthalpy= 0.169234

Biocyclo[4.1.0]heptane $\mathrm{C3}$ radical:

Charge $=\mathbf{0}$ Multiplicity $=2$

$\begin{array}{rrrr}\mathrm{C} & -1.159795 & -0.262997 & -0.566903 \\ \mathrm{C} & -1.232723 & -0.038975 & 0.935373 \\ \mathrm{C} & 0.139029 & -0.143727 & 1.628907 \\ \mathrm{C} & 1.219568 & 0.608062 & 0.915300 \\ \mathrm{C} & 1.177202 & 0.921503 & -0.549879 \\ \mathrm{C} & 0.056320 & 0.230506 & -1.316293 \\ \mathrm{C} & -0.188176 & -1.254716 & -1.167321 \\ \mathrm{H} & 0.053850 & 0.210849 & 2.665883 \\ \mathrm{H} & 0.407722 & -1.213402 & 1.721101 \\ \mathrm{H} & 2.107239 & 0.898355 & 1.472513 \\ \mathrm{H} & 2.147935 & 0.680315 & -1.015404 \\ \mathrm{H} & 1.067496 & 2.013407 & -0.692378 \\ \mathrm{H} & -0.117375 & 0.641371 & -2.309448 \\ \mathrm{H} & -2.116603 & -0.165198 & -1.077858 \\ \mathrm{H} & -1.649941 & 0.961616 & 1.117398 \\ \mathrm{H} & -1.928985 & -0.755980 & 1.390976 \\ \mathrm{H} & 0.463131 & -1.816470 & -0.502541 \\ \mathrm{H} & -0.503023 & -1.812806 & -2.045347 \\ & & & \\ \text { HF }=\mathbf{- 2 7 3 . 2 8 1 9 5 7 6} & & \end{array}$

Thermal correction to Enthalpy= $\mathbf{0 . 1 6 9 0 0 7}$

Biocyclo[4.1.0]heptane $\mathrm{C} 7$ radical:

Charge $=0$ Multiplicity $=2$

$\begin{array}{lrrr}\text { C } & -1.219226 & -0.248157 & -0.580250 \\ \text { C } & -1.225276 & -0.225200 & 0.944523 \\ \text { C } & 0.192595 & -0.212662 & 1.542425 \\ \text { C } & 1.055396 & 0.880078 & 0.894835 \\ \text { C } & 1.316074 & 0.608276 & -0.598699\end{array}$




$\begin{array}{lrrr}\mathrm{C} & 0.048356 & 0.195614 & -1.358990 \\ \mathrm{C} & -0.406522 & -1.204609 & -1.341666 \\ \mathrm{H} & 0.127941 & -0.053458 & 2.625782 \\ \mathrm{H} & 0.672301 & -1.192151 & 1.401401 \\ \mathrm{H} & 2.013588 & 0.972203 & 1.421796 \\ \mathrm{H} & 0.541530 & 1.845680 & 1.006281 \\ \mathrm{H} & 2.075877 & -0.180515 & -0.693560 \\ \mathrm{H} & 1.746459 & 1.503474 & -1.063414 \\ \mathrm{H} & -0.157360 & 0.773437 & -2.261999 \\ \mathrm{H} & -2.160305 & 0.068604 & -1.036404 \\ \mathrm{H} & -1.752765 & 0.680445 & 1.277568 \\ \mathrm{H} & -1.797279 & -1.079503 & 1.332255 \\ \mathrm{H} & 0.121626 & -2.098260 & -1.022773 \\ & & & \\ \text { HF }=\mathbf{- 2 7 3 . 2 6 6 0 3 5} & & \\ \text { Thermal correction to } & \end{array}$

Thermal correction to Enthalpy= 0.169589

Octahydro-pentalene:

\section{Charge $=0$ Multiplicity $=1$}

$\begin{array}{rrrr}\text { C } & -1.322232 & -1.206885 & -0.207357 \\ \mathrm{C} & -0.000003 & -0.788038 & 0.476589 \\ \mathrm{C} & 0.000002 & 0.788040 & 0.476589 \\ \mathrm{C} & -1.322207 & 1.206887 & -0.207386 \\ \mathrm{C} & -2.256939 & 0.000012 & -0.005308 \\ \mathrm{C} & 1.322208 & -1.206888 & -0.207382 \\ \mathrm{C} & 2.256940 & -0.000014 & -0.005312 \\ \mathrm{C} & 1.322231 & 1.206887 & -0.207353 \\ \mathrm{H} & -0.000014 & 1.148526 & 1.512849 \\ \mathrm{H} & 0.000008 & -1.148522 & 1.512850 \\ \mathrm{H} & 1.154377 & -1.361437 & -1.282563 \\ \mathrm{H} & 1.735082 & -2.141319 & 0.189503 \\ \mathrm{H} & 3.116911 & -0.000013 & -0.685542 \\ \mathrm{H} & 2.653715 & -0.000033 & 1.019483 \\ \mathrm{H} & 1.735119 & 2.141294 & 0.189572 \\ \mathrm{H} & 1.154413 & 1.361478 & -1.282530 \\ \mathrm{H} & -1.154370 & 1.361429 & -1.282568 \\ \mathrm{H} & -1.735080 & 2.141320 & 0.189494 \\ \mathrm{H} & -3.116914 & 0.000013 & -0.685535 \\ \mathrm{H} & -2.653712 & 0.000027 & 1.019488 \\ \mathrm{H} & -1.735115 & -2.141297 & 0.189562 \\ & & & \\ \text { HF }= & -\mathbf{3 1 3 . 2 8 9 2 5 7 4} & & \\ \mathrm{Th} & & \end{array}$

Thermal correction to Enthalpy= $\mathbf{0 . 2 1 4 4 3 7}$ 
Octahydro-pentalene $\mathrm{C} 1$ radical:

Charge $=0$ Multiplicity $=2$

$\begin{array}{lrrr}\text { C } & -0.819402 & 0.488219 & -1.536268 \\ \mathrm{C} & -0.773909 & 0.418616 & 0.007191 \\ \mathrm{C} & 0.692497 & 0.205438 & 0.305243 \\ \mathrm{C} & 1.544968 & 0.465387 & -0.902505 \\ \mathrm{C} & 0.551171 & 1.095144 & -1.914133 \\ \mathrm{C} & -1.464478 & -0.743736 & 0.756345 \\ \mathrm{C} & -0.542166 & -0.992895 & 1.971611 \\ \mathrm{C} & 0.891979 & -0.781680 & 1.418230 \\ \mathrm{H} & -1.134229 & 1.378613 & 0.421670 \\ \mathrm{H} & 1.960065 & -0.479829 & -1.297654 \\ \mathrm{H} & 2.413485 & 1.113081 & -0.710909 \\ \mathrm{H} & 0.828678 & 0.911885 & -2.958062 \\ \mathrm{H} & 0.523499 & 2.183490 & -1.772871 \\ \mathrm{H} & -1.663555 & 1.066565 & -1.928599 \\ \mathrm{H} & -0.899591 & -0.529062 & -1.944240 \\ \mathrm{H} & -1.478337 & -1.634342 & 0.112635 \\ \mathrm{H} & -2.500167 & -0.531183 & 1.044738 \\ \mathrm{H} & -0.685726 & -1.980300 & 2.424163 \\ \mathrm{H} & -0.749113 & -0.246924 & 2.750020 \\ \mathrm{H} & 1.595604 & -0.448896 & 2.195858 \\ \mathrm{H} & 1.305420 & -1.730059 & 1.028966 \\ & & & \\ \text { HF }= & \mathbf{- 3 1 2 . 6 2 9 9 6 0 9} & & \\ \mathrm{H} & & \end{array}$

Thermal correction to Enthalpy $=\mathbf{0 . 2 0 0 1 4 3}$

Octahydro-pentalene $\mathrm{C} 2$ radical:

Charge $=0$ Multiplicity $=2$

$\begin{array}{lrrr}\mathrm{C} & -0.731519 & 0.279320 & -1.528855 \\ \mathrm{C} & -0.770906 & 0.509051 & 0.001804 \\ \mathrm{C} & 0.729533 & 0.444443 & 0.446771 \\ \mathrm{C} & 1.512658 & 0.679654 & -0.804868 \\ \mathrm{C} & 0.618909 & 0.871434 & -1.993201 \\ \mathrm{C} & -1.522346 & -0.568268 & 0.832294 \\ \mathrm{C} & -0.490778 & -1.096902 & 1.856265 \\ \mathrm{C} & 0.861106 & -0.940192 & 1.141991 \\ \mathrm{H} & -1.174468 & 1.508986 & 0.202140 \\ \mathrm{H} & 0.942553 & 1.211696 & 1.209579 \\ \mathrm{H} & 2.588619 & 0.826388 & -0.828404 \\ \mathrm{H} & 0.998934 & 0.398467 & -2.909661 \\ \mathrm{H} & 0.505321 & 1.944744 & -2.236859 \\ \mathrm{H} & -1.592533 & 0.717257 & -2.046325 \\ \mathrm{H} & -0.744006 & -0.798424 & -1.737846\end{array}$




$\begin{array}{lrrr}\mathrm{H} & -1.850776 & -1.385756 & 0.177588 \\ \mathrm{H} & -2.421902 & -0.175670 & 1.318742 \\ \mathrm{H} & -0.695415 & -2.125424 & 2.175288 \\ \mathrm{H} & -0.503091 & -0.470393 & 2.758536 \\ \mathrm{H} & 1.721734 & -0.996361 & 1.817963 \\ \mathrm{H} & 0.985086 & -1.726746 & 0.386056\end{array}$

$\mathrm{HF}=\mathbf{- 3 1 2 . 6 2 5 2 5 8 9}$

Thermal correction to Enthalpy $=\mathbf{0 . 1 9 9 8 6 5}$

Octahydro-pentalene $\mathrm{C} 3$ radical:

Charge $=0$ Multiplicity $=2$

$\begin{array}{rrrr}\text { C } & -0.847222 & 0.590469 & -1.549490 \\ \mathrm{C} & -0.819720 & 0.561572 & 0.001703 \\ \mathrm{C} & 0.698555 & 0.561580 & 0.428903 \\ \mathrm{C} & 1.530907 & 0.590461 & -0.880352 \\ \mathrm{C} & 0.551966 & 0.258715 & -1.961707 \\ \mathrm{C} & -1.424371 & -0.713313 & 0.639456 \\ \mathrm{C} & -0.518808 & -1.013476 & 1.843851 \\ \mathrm{C} & 0.881934 & -0.713292 & 1.288405 \\ \mathrm{H} & -1.328106 & 1.442533 & 0.409518 \\ \mathrm{H} & 0.919685 & 1.442553 & 1.041969 \\ \mathrm{H} & 2.385319 & -0.100799 & -0.847941 \\ \mathrm{H} & 1.971062 & 1.593026 & -1.039402 \\ \mathrm{H} & 0.840527 & 0.045140 & -2.987260 \\ \mathrm{H} & -1.139847 & 1.593040 & -1.914716 \\ \mathrm{H} & -1.593178 & -0.100780 & -1.967382 \\ \mathrm{H} & -1.362966 & -1.547450 & -0.073546 \\ \mathrm{H} & -2.480942 & -0.593225 & 0.905080 \\ \mathrm{H} & -0.624915 & -2.037235 & 2.220981 \\ \mathrm{H} & -0.752414 & -0.332493 & 2.674039 \\ \mathrm{H} & 1.644952 & -0.593179 & 2.066028 \\ \mathrm{H} & 1.201370 & -1.547431 & 0.648015 \\ & & & \\ \text { HF }= & -\mathbf{3 1 2 . 6 2 4 5 8 0 7} & & \\ \mathrm{Th} & & & \end{array}$

Thermal correction to Enthalpy= 0.199817

Bicyclo[4.2.0]octane:

Charge $=0$ Multiplicity $=1$
C $\quad-1.337618$
$-0.706757 \quad-0.257325$
$\begin{array}{llll}\text { C } & -1.323699 & -0.722399 & 1.278404\end{array}$
$\begin{array}{llll}\text { C } & 0.120310 & -0.697502 & 1.801135\end{array}$
$\begin{array}{llll}\text { C } & 0.845500 & 0.586480 & 1.366100\end{array}$
$\begin{array}{llll}\text { C } & 0.705260 & 0.887951 & -0.128847\end{array}$ 


$\begin{array}{rrrr}\mathrm{C} & -0.639814 & 0.535196 & -0.845064 \\ \mathrm{C} & 1.446029 & 0.057163 & -1.215734 \\ \mathrm{C} & 0.194966 & 0.225791 & -2.121274 \\ \mathrm{H} & -1.871449 & 0.148363 & 1.669648 \\ \mathrm{H} & -1.847537 & -1.613232 & 1.648126 \\ \mathrm{H} & 0.651978 & -1.581768 & 1.420186 \\ \mathrm{H} & 0.136354 & -0.775822 & 2.895681 \\ \mathrm{H} & 1.908326 & 0.542235 & 1.642780 \\ \mathrm{H} & 0.417492 & 1.427837 & 1.931116 \\ \mathrm{H} & 0.930033 & 1.948349 & -0.302675 \\ \mathrm{H} & -1.357586 & 1.361597 & -0.920251 \\ \mathrm{H} & -2.364880 & -0.765766 & -0.639865 \\ \mathrm{H} & -0.832187 & -1.618132 & -0.608566 \\ \mathrm{H} & 1.594176 & -0.985883 & -0.914962 \\ \mathrm{H} & 2.401435 & 0.447112 & -1.582847 \\ \mathrm{H} & 0.286363 & 1.100648 & -2.774223 \\ & & & \\ \text { HF }=\mathbf{- 3 1 3 . 2 6 4 1 8 5 7} & & \\ \text { Ther }\end{array}$

Thermal correction to Enthalpy $=0.214007$

\section{Bicyclo[4.2.0]octane $\mathrm{C} 1$ radical:}

\section{Charge $=0$ Multiplicity $=2$}

$\begin{array}{lrrr}\mathrm{C} & -0.729817 & 0.719424 & -0.447755 \\ \mathrm{C} & -0.713810 & 0.900333 & 1.084549 \\ \mathrm{C} & 0.737233 & 0.961794 & 1.597099 \\ \mathrm{C} & 1.519723 & -0.318836 & 1.250777 \\ \mathrm{C} & 1.584988 & -0.568767 & -0.281414 \\ \mathrm{C} & 0.250604 & -0.333535 & -0.899674 \\ \mathrm{C} & -0.802196 & -1.200988 & -1.542977 \\ \mathrm{C} & -1.864212 & -0.140767 & -1.096748 \\ \mathrm{H} & 1.246318 & 1.833255 & 1.157959 \\ \mathrm{H} & 0.743636 & 1.114561 & 2.684006 \\ \mathrm{H} & 1.026514 & -1.174720 & 1.732966 \\ \mathrm{H} & 2.536040 & -0.266936 & 1.662322 \\ \mathrm{H} & 1.957594 & -1.579637 & -0.492558 \\ \mathrm{H} & 2.329421 & 0.128663 & -0.705818 \\ \mathrm{H} & -0.600334 & 1.706808 & -0.922577 \\ \mathrm{H} & -1.271891 & 1.797717 & 1.383118 \\ \mathrm{H} & -1.216504 & 0.043059 & 1.555750 \\ \mathrm{H} & -0.929962 & -2.205943 & -1.109970 \\ \mathrm{H} & -0.716944 & -1.335314 & -2.632783 \\ \mathrm{H} & -2.400436 & 0.345122 & -1.917628 \\ \mathrm{H} & -2.598524 & -0.518587 & -0.377927\end{array}$


$H F=-312.6028826$

Thermal correction to Enthalpy $=0.199755$

Bicyclo[4.2.0]octane $\mathrm{C} 2$ radical:

Charge $=0$ Multiplicity $=2$

$\begin{array}{rrrr}\text { C } & -0.661810 & 0.799183 & -0.467193 \\ \mathrm{C} & -0.679741 & 0.775832 & 1.068459 \\ \mathrm{C} & 0.739852 & 0.862759 & 1.646119 \\ \mathrm{C} & 1.570840 & -0.365994 & 1.229783 \\ \mathrm{C} & 1.528096 & -0.610775 & -0.248443 \\ \mathrm{C} & 0.349719 & -0.232340 & -1.066350 \\ \mathrm{C} & -0.870288 & -1.219133 & -1.260175 \\ \mathrm{C} & -1.817327 & 0.002268 & -1.132703 \\ \mathrm{H} & 1.229648 & 1.776679 & 1.281414 \\ \mathrm{H} & 0.705226 & 0.935627 & 2.740267 \\ \mathrm{H} & 1.182106 & -1.245726 & 1.780679 \\ \mathrm{H} & 2.611814 & -0.256880 & 1.564783 \\ \mathrm{H} & 2.308644 & -1.220340 & -0.700681 \\ \mathrm{H} & 0.668835 & 0.093348 & -2.066570 \\ \mathrm{H} & -0.523149 & 1.830625 & -0.814707 \\ \mathrm{H} & -1.307381 & 1.594946 & 1.443305 \\ \mathrm{H} & -1.146031 & -0.155247 & 1.424352 \\ \mathrm{H} & -0.967415 & -1.912733 & -0.419021 \\ \mathrm{H} & -0.897141 & -1.794440 & -2.191073 \\ \mathrm{H} & -2.086827 & 0.410982 & -2.112639 \\ \mathrm{H} & -2.734389 & -0.127639 & -0.547087 \\ & & & \\ \text { HF }= & \mathbf{- 3 1 2 . 6 0 2 0 4 7} & & \\ \mathrm{T} & & \end{array}$

Thermal correction to Enthalpy $=0.199511$

Bicyclo[4.2.0]octane C3radical:

Charge $=0$ Multiplicity $=2$

$\begin{array}{lrcr}\mathrm{C} & -0.671218 & 0.816132 & -0.435817 \\ \mathrm{C} & -0.729326 & 0.767430 & 1.100769 \\ \mathrm{C} & 0.676022 & 0.758895 & 1.738027 \\ \mathrm{C} & 1.485459 & -0.383370 & 1.209883 \\ \mathrm{C} & 1.627130 & -0.547395 & -0.270874 \\ \mathrm{C} & 0.349549 & -0.196983 & -1.053224 \\ \mathrm{C} & -0.818870 & -1.212255 & -1.189700 \\ \mathrm{C} & -1.801707 & -0.012311 & -1.112253 \\ \mathrm{H} & 1.172053 & 1.723320 & 1.511123 \\ \mathrm{H} & 0.596093 & 0.709610 & 2.830638 \\ \mathrm{H} & 2.158215 & -0.923867 & 1.871006 \\ \mathrm{H} & 1.955271 & -1.565378 & -0.524979\end{array}$




$\begin{array}{rrrr}\mathrm{H} & 2.440821 & 0.115631 & -0.628259 \\ \mathrm{H} & 0.623895 & 0.146986 & -2.058292 \\ \mathrm{H} & -0.557378 & 1.855043 & -0.769968 \\ \mathrm{H} & -1.318271 & 1.615211 & 1.474716 \\ \mathrm{H} & -1.252264 & -0.141437 & 1.426094 \\ \mathrm{H} & -0.897225 & -1.861407 & -0.310618 \\ \mathrm{H} & -0.841904 & -1.838932 & -2.087665 \\ \mathrm{H} & -2.050890 & 0.369498 & -2.108303 \\ \mathrm{H} & -2.730647 & -0.145145 & -0.546363 \\ & & & \\ \text { HF= -312.598001 } & & \end{array}$

Thermal correction to Enthalpy= 0.199374

Bicyclo[4.2.0]octane $\mathrm{C7}$ radical:

Charge $=\mathbf{0}$ Multiplicity $=2$

$\begin{array}{rrrr}\text { C } & -0.756578 & 0.766721 & -0.505926 \\ \mathrm{C} & -0.721996 & 0.863596 & 1.030623 \\ \mathrm{C} & 0.701749 & 0.853948 & 1.605566 \\ \mathrm{C} & 1.432147 & -0.433357 & 1.197033 \\ \mathrm{C} & 1.600615 & -0.508072 & -0.327514 \\ \mathrm{C} & 0.297611 & -0.243234 & -1.098532 \\ \mathrm{C} & -0.827412 & -1.241286 & -1.175952 \\ \mathrm{C} & -1.886406 & -0.182945 & -1.028259 \\ \mathrm{H} & 1.265440 & 1.727732 & 1.245000 \\ \mathrm{H} & 0.659733 & 0.938324 & 2.699018 \\ \mathrm{H} & 0.856774 & -1.299327 & 1.554595 \\ \mathrm{H} & 2.416782 & -0.488652 & 1.678513 \\ \mathrm{H} & 2.014523 & -1.481008 & -0.625910 \\ \mathrm{H} & 2.340367 & 0.247177 & -0.629984 \\ \mathrm{H} & 0.571000 & 0.079557 & -2.120744 \\ \mathrm{H} & -0.729160 & 1.766581 & -0.954626 \\ \mathrm{H} & -1.269081 & 1.757881 & 1.356392 \\ \mathrm{H} & -1.257440 & 0.003638 & 1.457560 \\ \mathrm{H} & -0.865877 & -2.275069 & -1.511985 \\ \mathrm{H} & -2.318199 & 0.145839 & -1.988417 \\ \mathrm{H} & -2.723237 & -0.374900 & -0.341644 \\ & & & \\ \text { HF }= & \mathbf{- 3 1 2 . 5 9 5 2 3 8 4} & & \\ \mathrm{H} & & \end{array}$

Thermal correction to Enthalpy= 0.199237

Bicyclo[1.1.1]pentane:

Charge $=0$ Multiplicity $=1$
C $\quad-0.547495$
$0.519302 \quad-0.984291$
$\begin{array}{llll}\text { C } & -0.541244 & 0.513953 & 0.572119\end{array}$ 


$\begin{array}{rrrr}\mathrm{C} & 1.012921 & 0.519208 & 0.492292 \\ \mathrm{C} & 0.541571 & -0.513475 & -0.572240 \\ \mathrm{C} & -0.465695 & -1.038834 & 0.492036 \\ \mathrm{H} & -1.171001 & 1.111718 & 1.237742 \\ \mathrm{H} & 1.171518 & -1.110979 & -1.237916 \\ \mathrm{H} & -0.040713 & -1.556860 & 1.359257 \\ \mathrm{H} & -1.355052 & -1.556953 & 0.115700 \\ \mathrm{H} & -1.443192 & 0.121439 & -1.474615 \\ \mathrm{H} & -0.198400 & 1.434566 & -1.475711 \\ \mathrm{H} & 1.552293 & 0.121703 & 1.359540 \\ \mathrm{H} & 1.484202 & 1.434440 & 0.116508\end{array}$

\section{$H F=\mathbf{- 1 9 5 . 2 6 0 5 4 0 6}$}

Thermal correction to Enthalpy= 0.122531

Bicyclo[1.1.1]pentane $\mathrm{C} 1$ radical:

Charge $=0$ Multiplicity $=2$

$\begin{array}{lrrr}\text { C } & -0.583859 & 0.558247 & -0.971364 \\ \text { C } & -0.549888 & 0.525543 & 0.576456 \\ \text { C } & 0.998135 & 0.557880 & 0.536830 \\ \text { C } & 0.490070 & -0.468958 & -0.514662 \\ \text { C } & -0.512027 & -1.022288 & 0.537713 \\ \text { H } & 1.124906 & -1.076144 & -1.180875 \\ \text { H } & 1.527165 & 0.152604 & 1.403323 \\ \text { H } & 1.467381 & 1.465841 & 0.149195 \\ \text { H } & -0.218954 & 1.466224 & -1.458445 \\ \text { H } & -1.474671 & 0.153245 & -1.458472 \\ \text { H } & -0.082382 & -1.531640 & 1.404217 \\ \text { H } & -1.398034 & -1.532673 & 0.151211 \\ & & & \\ \text { HF }=\mathbf{- 1 9 4 . 5 8 4 1 6 9} & & \end{array}$

Thermal correction to Enthalpy= 0.109283

Bicyclo[1.1.1]pentane $\mathrm{C} 2$ radical:

Charge $=\mathbf{0}$ Multiplicity $=\mathbf{2}$

$\begin{array}{rrrr}\mathrm{C} & -0.532540 & 0.488116 & -0.952442 \\ \mathrm{C} & -0.551547 & 0.477004 & 0.613424 \\ \mathrm{C} & 1.009493 & 0.459319 & 0.543527 \\ \mathrm{C} & 0.543747 & -0.570374 & -0.535760 \\ \mathrm{C} & -0.487004 & -1.043159 & 0.486580 \\ \mathrm{H} & 1.169731 & -1.172242 & -1.198347 \\ \mathrm{H} & -1.183644 & 1.078179 & 1.270815 \\ \mathrm{H} & 1.533400 & 0.053038 & 1.413154 \\ \mathrm{H} & 1.488523 & 1.371348 & 0.168860\end{array}$




$\begin{array}{rrrr}\text { H } & -0.149998 & 1.405218 & -1.423695 \\ \text { H } & -1.425593 & 0.117379 & -1.465723 \\ \text { H } & -1.325312 & -1.718358 & 0.302967 \\ & & & \\ \text { HF }= & -\mathbf{1 9 4 . 5 8 2 1 0 6 5} & & \end{array}$

Thermal correction to Enthalpy $=0.108709$

Tricyclo[1.1.1.01,3]pentane:

Charge $=0$ Multiplicity $=1$

$\begin{array}{lrrr}\mathrm{C} & -0.466298 & 0.506831 & -1.099181 \\ \mathrm{C} & -0.453015 & 0.492833 & 0.419500 \\ \mathrm{C} & 1.060032 & 0.506787 & 0.549360 \\ \mathrm{C} & 0.452930 & -0.492639 & -0.419315 \\ \mathrm{C} & -0.593682 & -1.013742 & 0.549678 \\ \mathrm{H} & -0.240670 & -1.473039 & 1.470505 \\ \mathrm{H} & -1.484653 & -1.472894 & 0.126794 \\ \mathrm{H} & -1.351465 & 0.116717 & -1.596925 \\ \mathrm{H} & -0.003503 & 1.356130 & -1.597186 \\ \mathrm{H} & 1.488213 & 0.116588 & 1.470182 \\ \mathrm{H} & 1.592275 & 1.356075 & 0.126378\end{array}$

$\mathrm{HF}=\mathbf{- 1 9 4 . 0 0 8 9 8 8 3}$

Thermal correction to Enthalpy= $\mathbf{0 . 0 9 8 7 6 8}$

Tricyclo[1.1.1.01,3]pentane $\mathrm{C} 2$ radical:

Charge $=0$ Multiplicity $=2$

$\begin{array}{lrrr}\mathrm{C} & -0.403354 & 0.557639 & -1.090991 \\ \mathrm{C} & -0.410177 & 0.536032 & 0.430375 \\ \mathrm{C} & 1.076995 & 0.510910 & 0.563000 \\ \mathrm{C} & 0.505072 & -0.459558 & -0.417071 \\ \mathrm{C} & -0.563101 & -0.979454 & 0.542193 \\ \mathrm{H} & -0.231059 & -1.456390 & 1.461148 \\ \mathrm{H} & -1.455094 & -1.420344 & 0.096879 \\ \mathrm{H} & -1.287858 & 0.173853 & -1.595341 \\ \mathrm{H} & 0.071361 & 1.406253 & -1.575218 \\ \mathrm{H} & 1.670040 & 0.303218 & 1.447494\end{array}$

$\mathrm{HF}=\mathbf{- 1 9 3 . 3 2 3 0 6 6 4}$

Thermal correction to Enthalpy $=0.084751$

Bicyclo[2.1.1]hexane:

Charge $=0$ Multiplicity $=1$
C $\quad-0.984598$
$0.062397 \quad-0.460072$
$\begin{array}{llll}\text { C } & -0.888135 & 0.127189 & 1.078540\end{array}$ 


\begin{tabular}{lrrr}
$\mathrm{C}$ & 0.666298 & 0.001280 & 1.234627 \\
$\mathrm{C}$ & 1.053275 & -0.102086 & -0.255299 \\
$\mathrm{C}$ & -0.008998 & -1.103106 & -0.797098 \\
$\mathrm{C}$ & 0.171447 & 1.009021 & -0.897246 \\
$\mathrm{H}$ & -1.982062 & 0.126385 & -0.903065 \\
$\mathrm{H}$ & -1.428912 & -0.692864 & 1.563954 \\
$\mathrm{H}$ & -1.277277 & 1.069778 & 1.479453 \\
$\mathrm{H}$ & 0.962484 & -0.887443 & 1.803035 \\
$\mathrm{H}$ & 1.113739 & 0.875311 & 1.720865 \\
$\mathrm{H}$ & 2.115752 & -0.204291 & -0.491897 \\
$\mathrm{H}$ & 0.085115 & -1.277639 & -1.873525 \\
$\mathrm{H}$ & -0.138802 & -2.056897 & -0.270866 \\
$\mathrm{H}$ & 0.209069 & 2.016241 & -0.464137 \\
$\mathrm{H}$ & 0.285164 & 1.063254 & -1.984526 \\
\multicolumn{4}{c}{} \\
HF = -234.6198654 & \\
Thermal correction to Enthalpy $=\mathbf{0 . 1 5 3 5 1 6}$
\end{tabular}

Bicyclo[2.1.1]hexane $\mathrm{C} 1$ radical:

Charge $=\mathbf{0}$ Multiplicity $=2$

$\begin{array}{lrrr}\text { C } & -0.651658 & 0.639356 & -1.044191 \\ \mathrm{C} & -0.649435 & 0.626401 & 0.520712 \\ \mathrm{C} & 0.863244 & 0.633767 & 0.850511 \\ \mathrm{C} & 1.339299 & -0.453529 & -0.192029 \\ \mathrm{C} & -0.031921 & -0.745981 & -0.791548 \\ \mathrm{C} & -0.963879 & -0.903477 & 0.422425 \\ \mathrm{H} & -1.300435 & 1.299817 & 1.090537 \\ \mathrm{H} & 1.065542 & 0.340971 & 1.886579 \\ \mathrm{H} & 1.322927 & 1.612971 & 0.677485 \\ \mathrm{H} & 1.791936 & -1.326674 & 0.288111 \\ \mathrm{H} & 2.049572 & -0.053366 & -0.922223 \\ \mathrm{H} & -2.006418 & -1.098781 & 0.155889 \\ \mathrm{H} & -0.645655 & -1.551888 & 1.245617 \\ \mathrm{H} & -0.049009 & 1.396429 & -1.557030 \\ \mathrm{H} & -1.662362 & 0.601304 & -1.460243 \\ & & & \\ \text { HF }= & \mathbf{- 2 3 3 . 9 3 9 5 5 1 6} & & \end{array}$

Thermal correction to Enthalpy= 0.140442

Bicyclo[2.1.1]hexane $\mathrm{C} 2$ radical:

Charge $=0$ Multiplicity $=2$
C $\quad-0.566291$
$0.657976-1.032846$
$\begin{array}{llll}\text { C } & -0.599828 & 0.689156 & 0.522651\end{array}$
$\begin{array}{llll}\text { C } & 0.907548 & 0.637420 & 0.883957\end{array}$ 


$\begin{array}{rrrr}\mathrm{C} & 1.316526 & -0.436547 & -0.104854 \\ \mathrm{C} & 0.025908 & -0.775540 & -0.789380 \\ \mathrm{C} & -0.963830 & -0.822541 & 0.436377 \\ \mathrm{H} & -1.220305 & 1.402284 & 1.071314 \\ \mathrm{H} & 1.078585 & 0.354502 & 1.933396 \\ \mathrm{H} & 1.410873 & 1.602824 & 0.723282 \\ \mathrm{H} & 2.195868 & -1.067244 & -0.040711 \\ \mathrm{H} & 0.022743 & -1.544373 & -1.564419 \\ \mathrm{H} & -2.002030 & -0.993564 & 0.131970 \\ \mathrm{H} & -0.702330 & -1.469862 & 1.281072 \\ \mathrm{H} & 0.063189 & 1.387074 & -1.554868 \\ \mathrm{H} & -1.566790 & 0.628816 & -1.476468 \\ & & & \\ \text { HF }=\mathbf{- 2 3 3 . 9 5 0 2 2 0 4} & & \end{array}$

Thermal correction to Enthalpy $=\mathbf{0 . 1 3 8 9 5 8}$

\section{Bicyclo[2.1.1]hexane C5 radical:}

Charge $=0$ Multiplicity $=2$

$\begin{array}{lrrr}\text { C } & -0.650558 & 0.642150 & -0.967486 \\ \mathrm{C} & -0.670175 & 0.640570 & 0.598173 \\ \mathrm{C} & 0.842775 & 0.622999 & 0.924239 \\ \mathrm{C} & 1.316114 & -0.456348 & -0.110456 \\ \mathrm{C} & -0.043763 & -0.787855 & -0.771133 \\ \mathrm{C} & -0.981121 & -0.844415 & 0.432038 \\ \mathrm{H} & -1.307453 & 1.317340 & 1.170706 \\ \mathrm{H} & 1.035908 & 0.323542 & 1.959125 \\ \mathrm{H} & 1.310602 & 1.599972 & 0.758728 \\ \mathrm{H} & 1.761821 & -1.331668 & 0.372371 \\ \mathrm{H} & 2.038633 & -0.060189 & -0.832784 \\ \mathrm{H} & -0.055779 & -1.536854 & -1.565357 \\ \mathrm{H} & -1.994098 & -1.239656 & 0.380942 \\ \mathrm{H} & -0.019892 & 1.395420 & -1.464772 \\ \mathrm{H} & -1.649383 & 0.629492 & -1.411210 \\ & & & \\ \text { HF }=\mathbf{- 2 3 3 . 9 4 3 5 5 2 6} & & \end{array}$

Thermal correction to Enthalpy $=\mathbf{0 . 1 3 9 6 6 0}$

Tricyclo[2.1.1.01,4]hexane:

\section{Charge $=\mathbf{0}$ Multiplicity $=1$}

$\begin{array}{lrrr}\text { C } & -0.568189 & -0.650903 & -1.187176 \\ \text { C } & -0.549920 & -0.642710 & 0.319848 \\ \text { C } & 0.910055 & -0.651521 & 0.864383 \\ \text { C } & 1.292200 & 0.574409 & 0.010294 \\ \text { C } & -0.141007 & 0.666922 & -0.594287\end{array}$




$\begin{array}{rrrr}\mathrm{C} & -1.186660 & 0.712330 & 0.490206 \\ \mathrm{H} & 1.442028 & -1.576879 & 0.625379 \\ \mathrm{H} & 0.956535 & -0.505518 & 1.947431 \\ \mathrm{H} & 2.059427 & 0.399937 & -0.749568 \\ \mathrm{H} & 1.570320 & 1.471396 & 0.571071 \\ \mathrm{H} & -1.551865 & -0.670530 & -1.656173 \\ \mathrm{H} & 0.175512 & -1.249332 & -1.711343 \\ \mathrm{H} & -0.970082 & 1.277139 & 1.395771 \\ \mathrm{H} & \mathbf{- 2 . 2 2 0 7 4 6} & 0.802625 & 0.157825 \\ & & & \\ \text { HF= -233.3227712 } & & \end{array}$

Thermal correction to Enthalpy $=\mathbf{0 . 1 2 8 4 3 2}$

Tricyclo[2.1.1.01,4]hexane C2 radical:

Charge $=\mathbf{0}$ Multiplicity $=2$

$\begin{array}{lrrr}\mathrm{C} & -0.467588 & -0.611099 & -1.232278 \\ \mathrm{C} & -0.489965 & -0.646669 & 0.268328 \\ \mathrm{C} & 0.957591 & -0.631039 & 0.864035 \\ \mathrm{C} & 1.260005 & 0.602555 & 0.073889 \\ \mathrm{C} & -0.095196 & 0.709546 & -0.576008 \\ \mathrm{C} & -1.193469 & 0.669983 & 0.474257 \\ \mathrm{H} & 1.530707 & -1.536124 & 0.618328 \\ \mathrm{H} & 0.972946 & -0.526782 & 1.958424 \\ \mathrm{H} & 2.029498 & 1.363536 & 0.160665 \\ \mathrm{H} & -1.434169 & -0.638372 & -1.735442 \\ \mathrm{H} & 0.316071 & -1.160089 & -1.750373 \\ \mathrm{H} & -1.025475 & 1.203382 & 1.407843 \\ \mathrm{H} & -2.217849 & 0.734783 & 0.107219\end{array}$

$\mathrm{HF}=\mathbf{- 2 3 2 . 6 5 3 6 9 0 3}$

Thermal correction to Enthalpy $=\mathbf{0 . 1 1 3 7 5 5}$

Tricyclo[2.1.1.01,4]hexane C5 radical:

Charge $=0$ Multiplicity $=2$

$\begin{array}{lrrr}\mathrm{C} & -0.585984 & -0.658606 & -1.207360 \\ \mathrm{C} & -0.586233 & -0.668207 & 0.259698 \\ \mathrm{C} & 0.866486 & -0.663398 & 0.845672 \\ \mathrm{C} & 1.253843 & 0.578138 & -0.019910 \\ \mathrm{C} & -0.179097 & 0.634391 & -0.648451 \\ \mathrm{C} & -1.216794 & 0.691790 & 0.447206 \\ \mathrm{H} & 1.424805 & -1.570765 & 0.599809 \\ \mathrm{H} & 0.903087 & -0.516513 & 1.928836 \\ \mathrm{H} & 2.034466 & 0.381476 & -0.759771 \\ \mathrm{H} & 1.523983 & 1.479109 & 0.538138\end{array}$




$\begin{array}{crrr}\text { H } & 0.025481 & -1.292541 & -1.842571 \\ \text { H } & -0.976768 & 1.248072 & 1.352345 \\ \text { H } & -2.248375 & 0.786517 & 0.122080 \\ & \\ \text { HF }= & -\mathbf{2 3 2 . 6 5 1 4 9 4 7} \\ \text { Thermal correction to Enthalpy= } & \mathbf{0 . 1 1 5 1 0 3}\end{array}$

Bicyclo[3.1.1]heptane:

Charge $=0$ Multiplicity $=1$

$\begin{array}{rrrr}\mathrm{C} & -0.746823 & -0.880531 & -1.011308 \\ \mathrm{C} & -0.720926 & -0.884763 & 0.541569 \\ \mathrm{C} & 0.740855 & -0.886131 & 1.026316 \\ \mathrm{C} & 1.470695 & 0.408985 & 0.589524 \\ \mathrm{C} & 0.929438 & 0.985804 & -0.742804 \\ \mathrm{C} & -0.565393 & 0.659077 & -0.917486 \\ \mathrm{C} & -1.282047 & 0.567553 & 0.463870 \\ \mathrm{H} & 1.323961 & 1.163072 & 1.371793 \\ \mathrm{H} & 2.553029 & 0.245611 & 0.532031 \\ \mathrm{H} & 1.481431 & 0.576618 & -1.600295 \\ \mathrm{H} & 1.091408 & 2.071657 & -0.759922 \\ \mathrm{H} & 0.784448 & -0.975440 & 2.119809 \\ \mathrm{H} & 1.244485 & -1.775338 & 0.622475 \\ \mathrm{H} & 0.025140 & -1.456342 & -1.538286 \\ \mathrm{H} & -1.731898 & -1.153938 & -1.405612 \\ \mathrm{H} & -1.011091 & 1.282600 & 1.249349 \\ \mathrm{H} & -2.370899 & 0.567121 & 0.347344 \\ \mathrm{H} & -1.319389 & -1.641776 & 1.060480 \\ \mathrm{H} & -1.025420 & 1.276198 & -1.697249 \\ & & & \\ \mathrm{HF}=\mathbf{- 2 7 3 . 9 4 2 9 5 4 1} & & \\ \mathrm{T} & & \end{array}$

Thermal correction to Enthalpy= $\mathbf{0 . 1 8 3 9 8 6}$

Bicyclo[3.1.1]heptane $\mathrm{C} 1$ radical:

Charge $=0$ Multiplicity $=2$

$\begin{array}{lrrr}\mathrm{C} & -0.772290 & -0.927890 & -0.999798 \\ \mathrm{C} & -0.702448 & -0.869351 & 0.523403 \\ \mathrm{C} & 0.704889 & -0.927199 & 1.057248 \\ \mathrm{C} & 1.453171 & 0.376474 & 0.610893 \\ \mathrm{C} & 0.897378 & 0.953508 & -0.717316 \\ \mathrm{C} & -0.600088 & 0.622524 & -0.892978 \\ \mathrm{C} & -1.315137 & 0.535025 & 0.497858 \\ \mathrm{H} & 1.320332 & 1.127014 & 1.397879 \\ \mathrm{H} & 2.532381 & 0.199054 & 0.539693 \\ \mathrm{H} & 1.448195 & 0.551919 & -1.578390\end{array}$




$\begin{array}{lrrr}\mathrm{H} & 1.049262 & 2.040409 & -0.731831 \\ \mathrm{H} & 0.726711 & -0.993205 & 2.153190 \\ \mathrm{H} & 1.212968 & -1.820200 & 0.671548 \\ \mathrm{H} & -0.000582 & -1.496410 & -1.532057 \\ \mathrm{H} & -1.762750 & -1.198096 & -1.384847 \\ \mathrm{H} & -1.040927 & 1.250323 & 1.280296 \\ \mathrm{H} & -2.404389 & 0.524753 & 0.380065 \\ \mathrm{H} & -1.074053 & 1.235895 & -1.671406 \\ & & & \\ \text { HF= -273.2712061 } & & \end{array}$

Thermal correction to Enthalpy $=\mathbf{0 . 1 7 0 6 5 0}$

Bicyclo[3.1.1]heptane $\mathrm{C} 2$ radical:

Charge $=0$ Multiplicity $=2$

$\begin{array}{lrrr}\text { C } & -0.683939 & -0.960971 & -0.968717 \\ \mathrm{C} & -0.713036 & -0.884238 & 0.602544 \\ \mathrm{C} & 0.685440 & -0.784869 & 1.106372 \\ \mathrm{C} & 1.507686 & 0.371609 & 0.615992 \\ \mathrm{C} & 0.951514 & 0.960055 & -0.716974 \\ \mathrm{C} & -0.525145 & 0.582828 & -0.942010 \\ \mathrm{C} & -1.303533 & 0.555752 & 0.403698 \\ \mathrm{H} & 1.510744 & 1.171249 & 1.378776 \\ \mathrm{H} & 2.563353 & 0.089864 & 0.503868 \\ \mathrm{H} & 1.539271 & 0.589576 & -1.567224 \\ \mathrm{H} & 1.063505 & 2.051584 & -0.712869 \\ \mathrm{H} & 1.084897 & -1.487235 & 1.831574 \\ \mathrm{H} & 0.110404 & -1.556562 & -1.432691 \\ \mathrm{H} & -1.656107 & -1.264833 & -1.373824 \\ \mathrm{H} & -1.073602 & 1.314431 & 1.160323 \\ \mathrm{H} & -2.386273 & 0.522591 & 0.241820 \\ \mathrm{H} & -1.315306 & -1.623737 & 1.138857 \\ \mathrm{H} & -0.954813 & 1.152078 & -1.774043 \\ & & & \\ \text { HF= -273.2787715 } & & \end{array}$

Thermal correction to Enthalpy= 0.169212

Bicyclo[3.1.1]heptane $\mathrm{C} 3$ radical:

Charge $=\mathbf{0}$ Multiplicity $=2$

$\begin{array}{lrrr}\text { C } & -0.586811 & -0.898044 & -1.012786 \\ \mathrm{C} & -0.685201 & -0.892051 & 0.539597 \\ \mathrm{C} & 0.720263 & -0.786162 & 1.150611 \\ \mathrm{C} & 1.512252 & 0.296513 & 0.474948 \\ \mathrm{C} & 0.910921 & 1.106336 & -0.637948 \\ \mathrm{C} & -0.529476 & 0.653711 & -0.921270\end{array}$




$\begin{array}{rrrr}\mathrm{C} & -1.314807 & 0.524929 & 0.415277 \\ \mathrm{H} & 2.531952 & 0.496553 & 0.795311 \\ \mathrm{H} & 1.516229 & 1.018002 & -1.557686 \\ \mathrm{H} & 0.920194 & 2.182420 & -0.388650 \\ \mathrm{H} & 0.640466 & -0.594228 & 2.235501 \\ \mathrm{H} & 1.236451 & -1.759129 & 1.066922 \\ \mathrm{H} & 0.263467 & -1.417671 & -1.471971 \\ \mathrm{H} & -1.515860 & -1.244276 & -1.478171 \\ \mathrm{H} & -1.102761 & 1.252993 & 1.208254 \\ \mathrm{H} & -2.396304 & 0.476755 & 0.249019 \\ \mathrm{H} & -1.275124 & -1.678374 & 1.022398 \\ \mathrm{H} & -0.981562 & 1.235563 & -1.731503 \\ & & & \\ \mathbf{H F}=\mathbf{- 2 7 3 . 2 8 3 8 8 6 7} & & \end{array}$

Thermal correction to Enthalpy $=\mathbf{0 . 1 6 9 0 3 3}$

Bicyclo[3.1.1]heptane C6 radical:

Charge $=\mathbf{0}$ Multiplicity $=2$

$\begin{array}{rrrr}\text { C } & -0.727718 & -0.878559 & -1.007199 \\ \mathrm{C} & -0.752425 & -0.922807 & 0.500937 \\ \mathrm{C} & 0.714588 & -0.902892 & 1.014531 \\ \mathrm{C} & 1.428414 & 0.402867 & 0.578554 \\ \mathrm{C} & 0.904054 & 0.977732 & -0.762792 \\ \mathrm{C} & -0.596588 & 0.624007 & -0.960912 \\ \mathrm{C} & -1.319391 & 0.533596 & 0.423960 \\ \mathrm{H} & 1.271604 & 1.156080 & 1.358828 \\ \mathrm{H} & 2.512623 & 0.247434 & 0.529665 \\ \mathrm{H} & 1.473912 & 0.577963 & -1.611891 \\ \mathrm{H} & 1.045230 & 2.067203 & -0.775687 \\ \mathrm{H} & 0.737629 & -0.985991 & 2.109798 \\ \mathrm{H} & 1.235738 & -1.786136 & 0.622351 \\ \mathrm{H} & -0.343617 & -1.599456 & -1.729050 \\ \mathrm{H} & -1.037995 & 1.245961 & 1.207728 \\ \mathrm{H} & -2.406415 & 0.533637 & 0.308123 \\ \mathrm{H} & -1.344491 & -1.692630 & 1.010948 \\ \mathrm{H} & -1.049819 & 1.232277 & -1.753294 \\ & & & \\ \text { HF }=\mathbf{- 2 7 3 . 2 7 0 6 8 3 2} & & \\ \text { Thermal } & & \end{array}$

Thermal correction to Enthalpy= 0.169916

Tricyclo[3.1.1.01,5]heptane:

Charge $=0$ Multiplicity $=1$
C $\quad-0.472500$
0.803303
$-1.318769$
C $\quad-0.536368$
0.832378
0.179836 


$\begin{array}{cccc}\mathrm{C} & 0.720683 & 0.776839 & 1.033329 \\ \mathrm{C} & 1.610590 & -0.327992 & 0.395608 \\ \mathrm{C} & 0.595362 & -1.338625 & -0.210370 \\ \mathrm{C} & -0.615957 & -0.511179 & -0.610052 \\ \mathrm{C} & -1.684448 & -0.116466 & 0.367828 \\ \mathrm{H} & 2.223780 & 0.105878 & -0.404172 \\ \mathrm{H} & 2.307074 & -0.787615 & 1.107223 \\ \mathrm{H} & 0.291803 & -2.077017 & 0.541738 \\ \mathrm{H} & 1.021105 & -1.901014 & -1.049076 \\ \mathrm{H} & 1.236903 & 1.741711 & 1.092520 \\ \mathrm{H} & 0.444712 & 0.504226 & 2.059273 \\ \mathrm{H} & -1.720912 & -0.667119 & 1.308129 \\ \mathrm{H} & -2.672284 & 0.163538 & -0.008907 \\ \mathrm{H} & 0.487654 & 1.020825 & -1.785508 \\ \mathrm{H} & -1.324004 & 1.187045 & -1.885673 \\ & & & \\ \mathbf{H F}=\mathbf{- 2 7 2 . 6 7 6 0 2 2 7} & & \end{array}$

Thermal correction to Enthalpy= $\mathbf{0 . 1 5 8 9 5 9}$

Tricyclo[3.1.1.01,5]heptane $\mathrm{C} 2$ radical:

\section{Charge $=0$ Multiplicity $=2$}

$\begin{array}{rrrr}\text { C } & -0.545715 & 0.761560 & -1.331255 \\ \mathrm{C} & -0.503846 & 0.876997 & 0.180603 \\ \mathrm{C} & 0.778578 & 0.841438 & 0.901045 \\ \mathrm{C} & 1.627092 & -0.335630 & 0.485040 \\ \mathrm{C} & 0.610941 & -1.322823 & -0.183692 \\ \mathrm{C} & -0.621398 & -0.500694 & -0.536386 \\ \mathrm{C} & -1.637024 & -0.076500 & 0.482390 \\ \mathrm{H} & 2.407683 & -0.033482 & -0.236128 \\ \mathrm{H} & 2.172134 & -0.791560 & 1.324349 \\ \mathrm{H} & 0.328121 & -2.113686 & 0.519578 \\ \mathrm{H} & 1.037162 & -1.820309 & -1.061293 \\ \mathrm{H} & 1.116394 & 1.611108 & 1.588430 \\ \mathrm{H} & -1.609901 & -0.581353 & 1.447192 \\ \mathrm{H} & -2.645370 & 0.185412 & 0.150629 \\ \mathrm{H} & 0.381125 & 0.963219 & -1.866860 \\ \mathrm{H} & -1.439106 & 1.114564 & -1.852367 \\ & & & \\ \text { HF }=\mathbf{- 2 7 2 . 0 1 1 4 9 1 1} & & \\ \text { Th } & & & \end{array}$

Thermal correction to Enthalpy= 0.144416

Tricyclo[3.1.1.01,5]heptane C3 radical:

Charge $=0$ Multiplicity $=2$

$\begin{array}{llll}\text { C } & -0.592890 & 0.802817 & -1.305786\end{array}$ 


$\begin{array}{rrrr}\text { C } & -0.503898 & 0.824307 & 0.190850 \\ \mathrm{C} & 0.828771 & 0.868046 & 0.922824 \\ \mathrm{C} & 1.524299 & -0.427264 & 0.573142 \\ \mathrm{C} & 0.700575 & -1.295809 & -0.349342 \\ \mathrm{C} & -0.583522 & -0.519728 & -0.599329 \\ \mathrm{C} & -1.551731 & -0.202506 & 0.500811 \\ \mathrm{H} & 2.510152 & -0.703211 & 0.943161 \\ \mathrm{H} & 0.484596 & -2.283193 & 0.089079 \\ \mathrm{H} & 1.220914 & -1.511228 & -1.296172 \\ \mathrm{H} & 1.414213 & 1.751460 & 0.622018 \\ \mathrm{H} & 0.677996 & 0.981238 & 2.008292 \\ \mathrm{H} & -1.437526 & -0.757169 & 1.432744 \\ \mathrm{H} & -2.593659 & 0.008883 & 0.246246 \\ \mathrm{H} & 0.308155 & 1.073567 & -1.857117 \\ \mathrm{H} & -1.514461 & 1.140481 & -1.787275 \\ & & & \\ \text { HF }=\mathbf{- 2 7 2 . 0 1 2 6 5 7 9} & & \end{array}$

Thermal correction to Enthalpy $=\mathbf{0 . 1 4 4 0 6 0}$

\section{Tricyclo[3.1.1.01,5]heptane C6 radical:}

\section{Charge $=0$ Multiplicity $=2$}

\begin{tabular}{lrrr}
$\mathrm{C}$ & -0.516005 & 0.796583 & -1.302937 \\
$\mathrm{C}$ & -0.607536 & 0.851320 & 0.198893 \\
$\mathrm{C}$ & 0.670890 & 0.810301 & 1.028206 \\
$\mathrm{C}$ & 1.535508 & -0.323585 & 0.395643 \\
$\mathrm{C}$ & 0.543127 & -1.345941 & -0.239468 \\
$\mathrm{C}$ & -0.689422 & -0.530667 & -0.613589 \\
$\mathrm{C}$ & -1.685135 & -0.124736 & 0.382004 \\
$\mathrm{H}$ & 2.173468 & 0.099705 & -0.388647 \\
$\mathrm{H}$ & 2.207362 & -0.792187 & 1.124997 \\
$\mathrm{H}$ & 0.259494 & -2.116967 & 0.485660 \\
$\mathrm{H}$ & 0.988508 & -1.858014 & -1.099188 \\
$\mathrm{H}$ & 1.203328 & 1.767482 & 1.032273 \\
$\mathrm{H}$ & 0.419012 & 0.575203 & 2.068410 \\
$\mathrm{H}$ & -1.848378 & -0.662855 & 1.313769 \\
$\mathrm{H}$ & 0.450911 & 1.007698 & -1.759483 \\
$\mathrm{H}$ & -1.362268 & 1.180284 & -1.870301 \\
& & & \\
HF= $\mathbf{- 2 7 2 . 0 0 9 8 6 5}$ & & \\
\hline
\end{tabular}

Thermal correction to Enthalpy= $\mathbf{0 . 1 4 5 9 8 1}$

Biocyclo[2.2.1]hetane:

Charge $=0$ Multiplicity $=1$

$\begin{array}{llll}\text { C } & -1.108051 & 0.386694 & -1.027807\end{array}$ 


$\begin{array}{lrrr}\mathrm{C} & -1.044104 & 0.224425 & 0.509919 \\ \mathrm{C} & 1.140826 & 0.301611 & -0.089909 \\ \mathrm{C} & 0.400752 & 0.439016 & -1.442068 \\ \mathrm{H} & -1.650106 & -0.437374 & -1.505320 \\ \mathrm{H} & -1.623941 & 1.314818 & -1.300033 \\ \mathrm{H} & 0.675264 & -0.357682 & -2.142705 \\ \mathrm{H} & 0.644427 & 1.392770 & -1.924381 \\ \mathrm{C} & 0.197556 & 1.074433 & 0.857874 \\ \mathrm{H} & 0.489738 & 1.004994 & 1.912790 \\ \mathrm{H} & 0.087244 & 2.133001 & 0.592720 \\ \mathrm{C} & -0.541287 & -1.203331 & 0.831806 \\ \mathrm{H} & -0.649982 & -1.422982 & 1.900258 \\ \mathrm{H} & -1.103078 & -1.969042 & 0.284949 \\ \mathrm{C} & 0.967741 & -1.149790 & 0.418519 \\ \mathrm{H} & 1.619149 & -1.340879 & 1.279225 \\ \mathrm{H} & 1.222656 & -1.887365 & -0.350852 \\ \mathrm{H} & -1.973378 & 0.492246 & 1.022056 \\ \mathrm{H} & 2.181406 & 0.639141 & -0.118708 \\ & & & \\ \text { HF }=\mathbf{- 2 7 3 . 9 6 8 4 9 0 6} & & \\ \text { Thermal correction to Enthalpy= } & \end{array}$

\section{Biocyclo[2.2.1]hetane $\mathrm{C} 1$ radical:}

\section{Charge $=\mathbf{0}$ Multiplicity $=2$}

$\begin{array}{lrrr}\mathrm{C} & -0.545968 & -0.877052 & -0.959981 \\ \mathrm{C} & -0.537181 & -0.862959 & 0.564549 \\ \mathrm{C} & 0.936002 & -0.883312 & 0.931011 \\ \mathrm{C} & 1.528157 & 0.062202 & -0.189002 \\ \mathrm{C} & 0.276335 & 0.443931 & -1.024407 \\ \mathrm{C} & -0.618785 & 1.398658 & -0.188983 \\ \mathrm{C} & -1.205764 & 0.449925 & 0.931032 \\ \mathrm{H} & -0.050923 & 2.237290 & 0.229470 \\ \mathrm{H} & -1.420750 & 1.820547 & -0.805661 \\ \mathrm{H} & -0.956378 & 0.787126 & 1.942382 \\ \mathrm{H} & -2.297910 & 0.390771 & 0.858285 \\ \mathrm{H} & -0.025051 & -1.732014 & -1.405115 \\ \mathrm{H} & -1.543077 & -0.787052 & -1.405102 \\ \mathrm{H} & 2.260732 & -0.471143 & -0.805693 \\ \mathrm{H} & 2.029974 & 0.941946 & 0.229455 \\ \mathrm{H} & 1.365040 & -1.889396 & 0.858243 \\ \mathrm{H} & 1.128496 & -0.510699 & 1.942363 \\ \mathrm{H} & 0.513075 & 0.824257 & -2.023931 \\ & & & \\ \text { HF= -273.2901162 } & & \end{array}$


Thermal correction to Enthalpy= 0.171275

Biocyclo[2.2.1]hetane $\mathrm{C} 2$ radical:

Charge $=\mathbf{0}$ Multiplicity $=\mathbf{2}$

$\begin{array}{rrrr}\text { C } & -0.601431 & -0.801258 & -0.955391 \\ \mathrm{C} & -0.600472 & -0.798969 & 0.590683 \\ \mathrm{C} & 0.920618 & -0.811756 & 0.893740 \\ \mathrm{C} & 1.532028 & 0.041251 & -0.265806 \\ \mathrm{C} & 0.266855 & 0.478633 & -1.074396 \\ \mathrm{C} & -0.513164 & 1.427770 & -0.208660 \\ \mathrm{C} & -1.149210 & 0.620029 & 0.896325 \\ \mathrm{H} & -0.363942 & 2.502849 & -0.180729 \\ \mathrm{H} & -0.884784 & 0.968644 & 1.906074 \\ \mathrm{H} & -2.251544 & 0.634995 & 0.843740 \\ \mathrm{H} & -0.123058 & -1.689265 & -1.385774 \\ \mathrm{H} & -1.600117 & -0.685043 & -1.390814 \\ \mathrm{H} & 2.199145 & -0.560059 & -0.896765 \\ \mathrm{H} & 2.106691 & 0.900665 & 0.093931 \\ \mathrm{H} & 1.309091 & -1.836288 & 0.875950 \\ \mathrm{H} & 1.143584 & -0.398319 & 1.883737 \\ \mathrm{H} & 0.491060 & 0.833267 & -2.084616 \\ \mathrm{H} & -1.157470 & -1.605637 & 1.076287 \\ & & & \\ \text { HF }=\mathbf{- 2 7 3 . 3 0 2 2 8 3 2} & & \end{array}$

Thermal correction to Enthalpy= 0.169832

Biocyclo[2.2.1]hetane $\mathrm{C} 7$ radical:

Charge $=0$ Multiplicity $=2$

$\begin{array}{lrrr}\text { C } & -0.597080 & -0.847370 & -0.914757 \\ \mathrm{C} & -0.606631 & -0.848877 & 0.596317 \\ \mathrm{C} & 0.926001 & -0.855837 & 0.889965 \\ \mathrm{C} & 1.516702 & -0.002986 & -0.285627 \\ \mathrm{C} & 0.250396 & 0.388500 & -1.109305 \\ \mathrm{C} & -0.548270 & 1.431485 & -0.281767 \\ \mathrm{C} & -1.138530 & 0.579269 & 0.892941 \\ \mathrm{H} & 0.090519 & 2.250189 & 0.068818 \\ \mathrm{H} & -1.347749 & 1.873319 & -0.885944 \\ \mathrm{H} & -0.818573 & 0.937650 & 1.878042 \\ \mathrm{H} & -2.233483 & 0.594496 & 0.876798 \\ \mathrm{H} & -0.560898 & -1.735494 & -1.540884 \\ \mathrm{H} & 2.205911 & -0.597479 & -0.895352 \\ \mathrm{H} & 2.066540 & 0.879609 & 0.062986 \\ \mathrm{H} & 1.318750 & -1.878362 & 0.870252 \\ \mathrm{H} & 1.154961 & -0.436517 & 1.877170\end{array}$




$$
\begin{array}{crcr}
\text { H } & 0.466404 & 0.696338 & -2.135565 \\
\mathrm{H} & -1.157910 & -1.648852 & 1.097083 \\
& & & \\
\text { HF }=\mathbf{- 2 7 3 . 2 9 2 8 5 8} &
\end{array}
$$

Thermal correction to Enthalpy= $\mathbf{0 . 1 7 0 4 5 6}$

Tricyclo[2.2.1.01,4]heptane:

\section{Charge $=0$ Multiplicity $=1$}

$\begin{array}{lrrr}\text { C } & -0.189321 & -0.765015 & -1.300389 \\ \mathrm{C} & -0.194448 & -0.785299 & 0.192905 \\ \mathrm{C} & 1.228428 & -0.750779 & 0.794241 \\ \mathrm{C} & 1.550127 & 0.548094 & -0.016835 \\ \mathrm{C} & 0.131119 & 0.529400 & -0.627937 \\ \mathrm{C} & -1.115279 & 1.208050 & -0.016945 \\ \mathrm{C} & -1.436974 & -0.090913 & 0.793993 \\ \mathrm{H} & -0.861687 & 2.057541 & 0.624466 \\ \mathrm{H} & -1.871002 & 1.545244 & -0.737854 \\ \mathrm{H} & -1.358151 & 0.053093 & 1.875842 \\ \mathrm{H} & -2.397506 & -0.580863 & 0.589403 \\ \mathrm{H} & 0.620706 & -1.283345 & -1.809332 \\ \mathrm{H} & -1.147565 & -0.845493 & -1.809432 \\ \mathrm{H} & 2.375918 & 0.493553 & -0.737687 \\ \mathrm{H} & 1.722220 & 1.417857 & 0.624449 \\ \mathrm{H} & 1.849430 & -1.632321 & 0.589876 \\ \mathrm{H} & 1.225713 & -0.586490 & 1.876070 \\ & & & \\ \text { HF }=\mathbf{- 2 7 2 . 6 3 8 4 8 3 5} & & \end{array}$

Thermal correction to Enthalpy= $\mathbf{0 . 1 5 7 8 8 5}$

Tricyclo[2.2.1.01,4]heptane $\mathrm{C} 2$ radical:

Charge $=0$ Multiplicity $=2$

$\begin{array}{lrrr}\text { C } & -0.232554 & -0.678548 & -1.329015 \\ \mathrm{C} & -0.158397 & -0.854193 & 0.164791 \\ \mathrm{C} & 1.227711 & -0.764354 & 0.682729 \\ \mathrm{C} & 1.606974 & 0.498945 & -0.084285 \\ \mathrm{C} & 0.151286 & 0.559063 & -0.608290 \\ \mathrm{C} & -1.028073 & 1.165208 & 0.190775 \\ \mathrm{C} & -1.382264 & -0.200419 & 0.858198 \\ \mathrm{H} & -0.686527 & 1.909894 & 0.915330 \\ \mathrm{H} & -1.809658 & 1.625950 & -0.426447 \\ \mathrm{H} & -1.303325 & -0.189279 & 1.948164 \\ \mathrm{H} & -2.351910 & -0.632703 & 0.585599 \\ \mathrm{H} & 0.535002 & -1.170669 & -1.920065 \\ \mathrm{H} & -1.226298 & -0.716863 & -1.772643\end{array}$




$\begin{array}{rrrr}\mathrm{H} & 2.377580 & 0.435731 & -0.865594 \\ \mathrm{H} & 1.865969 & 1.345014 & 0.566825 \\ \mathrm{H} & 1.491067 & -0.961285 & 1.719415\end{array}$

HF = -271.9757409

Thermal correction to Enthalpy $=\mathbf{0 . 1 4 4 2 0 6}$

Tricyclo[2.2.1.01,4]heptane $\mathrm{C7}$ radical:

Charge $=0$ Multiplicity $=2$

$\begin{array}{lrrr}\mathrm{C} & 0.000000 & 0.000000 & 0.000000 \\ \mathrm{C} & 0.000000 & 0.000000 & 1.546788 \\ \mathrm{C} & 1.327018 & 0.000000 & 2.357058 \\ \mathrm{C} & 1.400742 & 1.570683 & 2.309071 \\ \mathrm{C} & 0.077235 & 1.645511 & 1.496515 \\ \mathrm{C} & 0.072813 & 1.551293 & -0.047394 \\ \mathrm{C} & -0.990103 & 0.889842 & 2.137386 \\ \mathrm{H} & 2.267730 & 1.958699 & 1.764499 \\ \mathrm{H} & 1.364275 & 2.065728 & 3.287495 \\ \mathrm{H} & 2.152329 & -0.499894 & 1.839611 \\ \mathrm{H} & 1.247185 & -0.428956 & 3.363703 \\ \mathrm{H} & -1.165598 & 0.930860 & 3.211200 \\ \mathrm{H} & -0.763826 & 2.068830 & -0.527429 \\ \mathrm{H} & 1.003522 & 1.920752 & -0.487472 \\ \mathrm{H} & -0.882766 & -0.465252 & -0.450009 \\ \mathrm{H} & 0.890791 & -0.481046 & -0.414093\end{array}$

$\mathrm{HF}=\mathbf{- 2 7 1 . 9 6 1 2 1 2}$

Thermal correction to Enthalpy $=0.144432$

Bicyclo[2.2.2] ]octane:

Charge $=0$ Multiplicity $=1$

$\begin{array}{lrrr}\mathrm{C} & -1.247414 & 0.248097 & -1.047218 \\ \mathrm{C} & -1.210351 & 0.000013 & 0.474140 \\ \mathrm{C} & 1.210320 & 0.000017 & -0.474180 \\ \mathrm{C} & 0.203918 & 0.244128 & -1.616529 \\ \mathrm{H} & -1.856798 & -0.525276 & -1.531602 \\ \mathrm{H} & -1.742233 & 1.205998 & -1.251487 \\ \mathrm{H} & 0.319314 & -0.534776 & -2.380821 \\ \mathrm{H} & 0.430549 & 1.198079 & -2.109217 \\ \mathrm{C} & -0.543296 & -1.362569 & 0.750167 \\ \mathrm{H} & -0.539960 & -1.558915 & 1.829752 \\ \mathrm{H} & -1.137946 & -2.162422 & 0.291112 \\ \mathrm{C} & 0.909602 & -1.361275 & 0.184918 \\ \mathrm{H} & 1.637330 & -1.551784 & 0.983803\end{array}$




$\begin{array}{lrrr}\text { H } & 1.040311 & -2.163775 & -0.551936 \\ \text { H } & 2.232238 & -0.000001 & -0.874371 \\ \text { H } & -2.232208 & 0.000003 & 0.874496 \\ \text { C } & 1.063797 & 1.117133 & 0.578552 \\ \text { H } & 1.797706 & 0.966183 & 1.380197 \\ \text { H } & 1.299065 & 2.086260 & 0.120795 \\ \text { C } & -0.386529 & 1.114440 & 1.150265 \\ \text { H } & -0.375742 & 0.956397 & 2.236092 \\ & & & \\ \text { HF }=\mathbf{- 3 1 3 . 2 8 9 9 4 3 6} & & \end{array}$

Thermal correction to Enthalpy $=\mathbf{0 . 2 1 5 2 8 3}$

Bicyclo[2.2.2]octane $\mathrm{C} 1$ radical:

Charge $=0$ Multiplicity $=2$

\begin{tabular}{rrrr} 
C & -0.603480 & -1.025283 & -1.118755 \\
$\mathrm{C}$ & -0.597883 & -1.024347 & 0.425471 \\
$\mathrm{C}$ & 0.861703 & -1.027964 & 0.931758 \\
$\mathrm{C}$ & 1.599925 & 0.267056 & 0.404802 \\
$\mathrm{C}$ & 0.597540 & 1.024676 & -0.427131 \\
$\mathrm{C}$ & 0.159129 & 0.254833 & -1.645086 \\
$\mathrm{C}$ & -0.565594 & 1.524707 & 0.389348 \\
$\mathrm{C}$ & -1.312929 & 0.244683 & 0.939656 \\
$\mathrm{H}$ & 0.877873 & -1.058093 & 2.028239 \\
$\mathrm{H}$ & 1.376012 & -1.931473 & 0.581492 \\
$\mathrm{H}$ & 1.955011 & 0.870817 & 1.248659 \\
$\mathrm{H}$ & 2.478822 & -0.012179 & -0.188897 \\
$\mathrm{H}$ & -2.359894 & 0.241701 & 0.612320 \\
$\mathrm{H}$ & -1.322614 & 0.249335 & 2.036575 \\
$\mathrm{H}$ & -1.247415 & 2.131598 & -0.218420 \\
$\mathrm{H}$ & -0.225159 & 2.151601 & 1.222351 \\
$\mathrm{H}$ & -1.119061 & -1.916678 & 0.796278 \\
$\mathrm{H}$ & -0.502746 & 0.857487 & -2.278701 \\
$\mathrm{H}$ & 1.017918 & -0.041373 & -2.259314 \\
$\mathrm{H}$ & -1.636383 & -1.035458 & -1.487899 \\
$\mathrm{H}$ & -0.122828 & -1.937448 & -1.493066 \\
& & & \\
HF = -312.6206161 & & \\
\hline
\end{tabular}

Thermal correction to Enthalpy= $\mathbf{0 . 2 0 1 8 2 1}$

Bicyclo[2.2.2] octane $\mathrm{C} 2$ radical:

Charge $=0$ Multiplicity $=2$
C $\quad-0.643268$
$-1.032154-1.141456$
$\begin{array}{llll}\text { C } & -0.592930 & -1.063452 & 0.401381\end{array}$
$\begin{array}{llll}\text { C } & 0.882833 & -1.057882 & 0.856507\end{array}$ 


$\begin{array}{lrrr}\text { C } & 1.595227 & 0.231012 & 0.345354 \\ \mathrm{C} & 0.594789 & 1.074009 & -0.494250 \\ \mathrm{C} & 0.052665 & 0.215369 & -1.597595 \\ \mathrm{C} & -0.578307 & 1.479134 & 0.441755 \\ \mathrm{C} & -1.298423 & 0.194655 & 0.953176 \\ \mathrm{H} & 0.928277 & -1.117753 & 1.950985 \\ \mathrm{H} & 1.386219 & -1.954681 & 0.474416 \\ \mathrm{H} & 1.962078 & 0.834812 & 1.185921 \\ \mathrm{H} & 2.467425 & -0.022671 & -0.267171 \\ \mathrm{H} & -2.349855 & 0.190753 & 0.639941 \\ \mathrm{H} & -1.300081 & 0.161737 & 2.049740 \\ \mathrm{H} & -1.275513 & 2.126702 & -0.101237 \\ \mathrm{H} & -0.185835 & 2.068161 & 1.281236 \\ \mathrm{H} & 1.091506 & 1.969141 & -0.884529 \\ \mathrm{H} & -1.093808 & -1.964611 & 0.776045 \\ \mathrm{H} & 0.154645 & 0.469815 & -2.648208 \\ \mathrm{H} & -1.690983 & -1.066178 & -1.489522 \\ \mathrm{H} & -0.169584 & -1.939377 & -1.556850 \\ & & & \\ \text { HF = } \mathbf{- 3 1 2 . 6 2 5 7 0 9 5} & & \\ \text { Thermal correction to Enthalpy= } & \mathbf{0 . 2 0 0 5 0 6}\end{array}$

\section{Tricyclo[2.2.2.01,4]octane:}

\section{Charge $=0$ Multiplicity $=1$}

$\begin{array}{lrrr}\mathrm{C} & -0.008563 & -0.803364 & -1.545992 \\ \mathrm{C} & -0.009565 & -0.768706 & 0.008396 \\ \mathrm{C} & 1.335841 & -0.795971 & 0.786951 \\ \mathrm{C} & 1.357421 & 0.777998 & 0.766384 \\ \mathrm{C} & 0.009659 & 0.768820 & -0.008226 \\ \mathrm{C} & 0.008319 & 0.770613 & -1.562941 \\ \mathrm{C} & -1.335110 & 0.812545 & 0.771158 \\ \mathrm{C} & -1.357257 & -0.761843 & 0.783775 \\ \mathrm{H} & 1.276577 & -1.240110 & 1.787082 \\ \mathrm{H} & 2.164926 & -1.276284 & 0.255132 \\ \mathrm{H} & 1.314957 & 1.248652 & 1.755151 \\ \mathrm{H} & 2.197860 & 1.220710 & 0.219972 \\ \mathrm{H} & -2.198049 & -1.212464 & 0.244163 \\ \mathrm{H} & -1.316871 & -1.214759 & 1.780947 \\ \mathrm{H} & -2.165793 & 1.279542 & 0.229785 \\ \mathrm{H} & -1.275309 & 1.278573 & 1.761254 \\ \mathrm{H} & -0.873554 & 1.233842 & -2.019587 \\ \mathrm{H} & 0.897596 & 1.217116 & -2.022196 \\ \mathrm{H} & -0.898967 & -1.258154 & -1.994509 \\ \mathrm{H} & 0.872158 & -1.277218 & -1.994221\end{array}$




\section{$H F=-311.9628081$}

Thermal correction to Enthalpy $=\mathbf{0 . 1 8 7 6 0 0}$

Tricyclo[2.2.2.01,4]octane $\mathrm{C} 2$ radical:

Charge $=0$ Multiplicity $=2$

$\begin{array}{lrrr}\mathrm{C} & -0.083227 & -0.756863 & -1.535039 \\ \mathrm{C} & -0.036336 & -0.795149 & -0.037625 \\ \mathrm{C} & 1.335517 & -0.864756 & 0.716132 \\ \mathrm{C} & 1.405765 & 0.705179 & 0.680086 \\ \mathrm{C} & 0.029340 & 0.746841 & -0.041747 \\ \mathrm{C} & -0.029599 & 0.759421 & -1.601754 \\ \mathrm{C} & -1.284547 & 0.823014 & 0.785596 \\ \mathrm{C} & -1.356292 & -0.747159 & 0.806472 \\ \mathrm{H} & 1.271025 & -1.295054 & 1.721799 \\ \mathrm{H} & 2.135755 & -1.380242 & 0.176304 \\ \mathrm{H} & 1.423059 & 1.188223 & 1.663418 \\ \mathrm{H} & 2.236529 & 1.110586 & 0.092151 \\ \mathrm{H} & -2.233488 & -1.178603 & 0.314923 \\ \mathrm{H} & -1.267628 & -1.196184 & 1.802068 \\ \mathrm{H} & -2.118106 & 1.312410 & 0.270062 \\ \mathrm{H} & -1.178096 & 1.290480 & 1.770935 \\ \mathrm{H} & -0.913120 & 1.257589 & -2.033600 \\ \mathrm{H} & 0.851541 & 1.192478 & -2.102920 \\ \mathrm{H} & -0.091191 & -1.524845 & -2.307867 \\ & & & \\ \text { HF = -311.2967296 } & & \\ \mathrm{T}\end{array}$

Thermal correction to Enthalpy $=\mathbf{0 . 1 7 3 1 2 0}$

Tricyclo[1.1.0.0 $\left.0^{2,4}\right]$ butane

\section{Charge $=0$ Multiplicity $=1$}

$\begin{array}{lrrr}\text { C } & -0.400217 & -0.337316 & -0.739377 \\ \text { C } & -0.400214 & -0.337387 & 0.739373 \\ \text { C } & 0.876589 & -0.227741 & -0.000032 \\ \text { C } & -0.077228 & 0.902673 & 0.000031 \\ \text { H } & -0.870519 & -0.735777 & -1.617669 \\ \text { H } & -0.870470 & -0.735938 & 1.617643 \\ \text { H } & 1.914016 & -0.501585 & -0.000021 \\ \text { H } & -0.166605 & 1.971929 & 0.000073\end{array}$

$\mathrm{HF}=\mathbf{- 1 5 4 . 6 3 6 6 6 1}$

Thermal correction to Enthalpy= 0.064610 
Tetrahedrane:

Charge $=0$ Multiplicity $=1$

$\begin{array}{lrrr}\mathrm{C} & 0.000000 & 0.000000 & 0.000000 \\ \mathrm{C} & 0.000000 & 0.000000 & 1.659950 \\ \mathrm{C} & 1.304050 & 0.000000 & 1.031016 \\ \mathrm{C} & 0.996251 & 1.262301 & 0.391211 \\ \mathrm{C} & -0.308890 & 1.263158 & 1.021223 \\ \mathrm{H} & -0.581494 & -0.737234 & 2.193324 \\ \mathrm{H} & -1.205509 & 1.853500 & 0.905690 \\ \mathrm{H} & 1.461213 & 1.850136 & -0.386053 \\ \mathrm{H} & 2.078095 & -0.741992 & 0.902331\end{array}$

$\mathrm{HF}=\mathbf{- 1 9 2 . 7 3 0 1 4 9 4}$

Thermal correction to Enthalpy $=\mathbf{0 . 0 7 2 3 2 3}$

Tetrahedrane $\mathbf{C 1}$ radical:

Charge $=0$ Multiplicity $=2$

$\begin{array}{lrrr}\mathrm{C} & 0.000000 & 0.000000 & 0.000000 \\ \mathrm{C} & 0.000000 & 0.000000 & 1.639476 \\ \mathrm{C} & 1.693612 & 0.000000 & 1.134106 \\ \mathrm{C} & 0.766091 & 1.031484 & 0.696295 \\ \mathrm{C} & 0.765368 & -1.031607 & 0.697197 \\ \mathrm{H} & 0.710157 & 2.105162 & 0.782864 \\ \mathrm{H} & -0.917634 & 0.000105 & -0.574337 \\ \mathrm{H} & 0.709842 & -2.105342 & 0.783235\end{array}$

HF $=\mathbf{- 1 9 2 . 0 3 7 9 8 2}$

Thermal correction to Enthalpy= $\mathbf{0 . 0 5 8 9 3 7}$

Prismane:

Charge $=0$ Multiplicity $=1$

$\begin{array}{lrrr}\mathrm{C} & 0.000000 & 0.000000 & 0.000000 \\ \mathrm{C} & 0.000000 & 0.000000 & 1.521738 \\ \mathrm{C} & 1.557961 & 0.000000 & 1.521517 \\ \mathrm{C} & 1.557970 & 0.001458 & -0.000080 \\ \mathrm{C} & 1.557462 & 1.318616 & 0.762308 \\ \mathrm{C} & -0.000230 & 1.318052 & 0.760762 \\ \mathrm{H} & -0.736340 & -0.398743 & 2.211557 \\ \mathrm{H} & 2.293820 & -0.397001 & -0.690554 \\ \mathrm{H} & 2.291413 & 2.117572 & 0.759842 \\ \mathrm{H} & 2.292559 & -0.398460 & 2.213433 \\ \mathrm{H} & -0.738080 & 2.113373 & 0.764066 \\ \mathrm{H} & -0.734208 & -0.397765 & -0.692609\end{array}$


$\mathrm{HF}=\mathbf{- 2 3 2 . 0 5 5 0 0 1 6}$

Thermal correction to Enthalpy $=\mathbf{0 . 1 0 2 7 2 6}$

Prismane $\mathrm{C} 1$ radical

Charge $=0$ Multiplicity $=2$

$\begin{array}{lrrr}\text { C } & 0.000000 & 0.000000 & 0.000000 \\ \mathrm{C} & 0.000000 & 0.000000 & 1.515401 \\ \mathrm{C} & 1.335970 & 0.000000 & 0.800111 \\ \mathrm{C} & 1.350418 & -1.541135 & 0.780079 \\ \mathrm{C} & 0.020380 & -1.563613 & 1.481385 \\ \mathrm{C} & 0.010810 & -1.541138 & -0.022184 \\ \mathrm{H} & 2.125215 & 0.746944 & 0.819152 \\ \mathrm{H} & -0.279857 & -2.266542 & -0.774470 \\ \mathrm{H} & 2.150811 & -2.266554 & 0.681245 \\ \mathrm{H} & -0.389324 & 0.746929 & -0.686820 \\ \mathrm{H} & -0.406573 & 0.741758 & 2.194263 \\ & & & \\ \text { HF }=\mathbf{- 2 3 1 . 3 7 6 5 7 1 8} & & \end{array}$

Thermal correction to Enthalpy= 0.089563<smiles>[C]1C2C3C1C23</smiles>

\section{Charge $=0$ Multiplicity $=1$}

$\begin{array}{rrrr}\mathrm{C} & 0.000000 & 0.000000 & 0.000000 \\ \mathrm{C} & 0.000000 & 0.000000 & 1.480576 \\ \mathrm{C} & 1.437807 & 0.000000 & 1.122457 \\ \mathrm{C} & 1.144984 & -0.939179 & 0.013995 \\ \mathrm{C} & 0.282200 & -1.410645 & 1.122330 \\ \mathrm{H} & -0.589070 & 0.485202 & 2.241444 \\ \mathrm{H} & 1.741075 & -1.421336 & -0.743342\end{array}$

$\mathrm{HF}=\mathbf{- 1 9 1 . 4 1 7 1 9 7 9}$

Thermal correction to Enthalpy= 0.047571

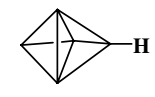

\section{C1 radical:}

\section{Charge $=\mathbf{0}$ Multiplicity $=\mathbf{2}$}

$\begin{array}{rrrr}\mathrm{C} & 0.000000 & 0.000000 & 0.000000 \\ \mathrm{C} & 0.000000 & 0.000000 & 1.466628 \\ \mathrm{C} & 1.431168 & 0.000000 & 1.146226 \\ \mathrm{C} & 1.140470 & -0.951313 & 0.041531 \\ \mathrm{C} & 0.256534 & -1.408098 & 1.145850 \\ \mathrm{H} & 1.739598 & -1.451367 & -0.705482\end{array}$


$\mathrm{HF}=\mathbf{- 1 9 0 . 7 1 5 8 9 2 9}$

Thermal correction to Enthalpy $=\mathbf{0 . 0 3 4 3 2 6}$

\section{Cubane:}

Charge $=0$ Multiplicity $=1$

$\begin{array}{lrrr}\mathrm{C} & 0.000000 & 0.000000 & 0.000000 \\ \mathrm{C} & 0.000000 & 0.000000 & 1.570468 \\ \mathrm{C} & 1.570457 & 0.000000 & 1.570605 \\ \mathrm{C} & 1.570238 & -1.570492 & 1.570413 \\ \mathrm{C} & -0.000328 & -1.570437 & 1.570523 \\ \mathrm{C} & -0.000246 & -1.570492 & -0.000020 \\ \mathrm{C} & 1.570212 & -1.570327 & -0.000075 \\ \mathrm{C} & 1.570568 & 0.000107 & 0.000063 \\ \mathrm{H} & -0.630443 & 0.630575 & -0.630616 \\ \mathrm{H} & -0.630735 & -2.200992 & -0.630662 \\ \mathrm{H} & -0.630576 & 0.630740 & 2.200779 \\ \mathrm{H} & -0.631120 & -2.200773 & 2.201013 \\ \mathrm{H} & 2.200944 & 0.630403 & 2.201345 \\ \mathrm{H} & 2.201316 & 0.630545 & -0.630367 \\ \mathrm{H} & 2.200764 & -2.201022 & -0.630454 \\ \mathrm{H} & 2.200737 & -2.201118 & 2.200923\end{array}$

$\mathrm{HF}=\mathbf{- 3 0 9 . 4 6 0 3 9 4 7}$

Thermal correction to Enthalpy= 0.139618

Cubane C1 radical:

Charge $=0$ Multiplicity $=2$

$\begin{array}{rrrr}\mathrm{C} & 0.000000 & 0.000000 & 0.000000 \\ \mathrm{C} & 0.000000 & 0.000000 & 1.554069 \\ \mathrm{C} & 1.552565 & 0.000000 & 1.622480 \\ \mathrm{C} & 1.571240 & -0.028962 & 0.050550 \\ \mathrm{C} & 1.524936 & -1.596665 & 0.094743 \\ \mathrm{C} & -0.043175 & -1.570499 & 0.050709 \\ \mathrm{C} & -0.071514 & -1.550966 & 1.622591 \\ \mathrm{C} & 1.500069 & -1.570499 & 1.663270 \\ \mathrm{H} & 2.186797 & 0.627872 & 2.251038 \\ \mathrm{H} & 2.231119 & 0.570210 & -0.580891 \\ \mathrm{H} & -0.600024 & 0.627889 & -0.661276 \\ \mathrm{H} & -0.727904 & -2.155953 & 2.250795 \\ \mathrm{H} & 2.103562 & -2.202042 & 2.319159 \\ \mathrm{H} & 2.153230 & -2.254684 & -0.506546 \\ \mathrm{H} & -0.671953 & -2.202028 & -0.580992 \\ & & & \\ \mathbf{H F}=\mathbf{- 3 0 8 . 7 8 5 9 1 2 8} & & \end{array}$


Thermal correction to Enthalpy $=0.126576$

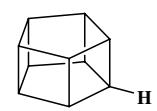

Charge $=0$ Multiplicity $=1$

\begin{tabular}{lrrr} 
C & -0.816795 & 1.020005 & -0.819988 \\
$\mathrm{C}$ & -0.812126 & 1.083257 & 0.739604 \\
$\mathrm{C}$ & 0.752773 & 1.062158 & -0.826138 \\
$\mathrm{C}$ & 0.792264 & -0.439726 & -1.248418 \\
$\mathrm{C}$ & 0.821631 & -1.304853 & 0.050039 \\
$\mathrm{C}$ & 0.800062 & -0.337271 & 1.275194 \\
$\mathrm{C}$ & 0.757155 & 1.125636 & 0.733039 \\
$\mathrm{C}$ & -0.777418 & -0.482003 & -1.241540 \\
$\mathrm{C}$ & -0.747829 & -1.346817 & 0.057127 \\
$\mathrm{C}$ & -0.769641 & -0.380317 & 1.281135 \\
$\mathrm{H}$ & -1.438872 & 1.718669 & -1.384831 \\
$\mathrm{H}$ & -1.431142 & 1.825795 & 1.249143 \\
$\mathrm{H}$ & 1.333529 & 1.794795 & -1.391985 \\
$\mathrm{H}$ & 1.396566 & -0.738089 & -2.108600 \\
$\mathrm{H}$ & 1.446438 & -2.200689 & 0.085322 \\
$\mathrm{H}$ & 1.410926 & -0.566153 & 2.151922 \\
$\mathrm{H}$ & 1.341600 & 1.900298 & 1.235580 \\
$\mathrm{H}$ & -1.372878 & -0.817075 & -2.094360 \\
$\mathrm{H}$ & -1.324027 & -2.274527 & 0.096899 \\
$\mathrm{H}$ & -1.362597 & -0.643429 & 2.160587 \\
& \multicolumn{3}{c}{} \\
HF= -386.9214984 & & \\
Thermal correction & to Enthalpy= & $\mathbf{0 . 1 7 7 1 9 1}$
\end{tabular}

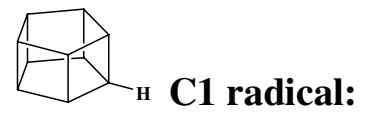

\section{Charge $=0$ Multiplicity $=2$}

$\begin{array}{lrrr}\text { C } & -0.838268 & 1.016560 & -0.797845 \\ \text { C } & -0.844889 & 1.075855 & 0.765279 \\ \text { C } & 0.729033 & 1.049862 & -0.778751 \\ \text { C } & 0.769293 & -0.451434 & -1.202215 \\ \text { C } & 0.796978 & -1.320428 & 0.096466 \\ \text { C } & 0.793520 & -0.351321 & 1.322199 \\ \text { C } & 0.731451 & 1.113102 & 0.782931 \\ \text { C } & -0.797907 & -0.490453 & -1.222815 \\ \text { C } & -0.779600 & -1.357145 & 0.079347 \\ \text { C } & -0.754855 & -0.374868 & 1.256440 \\ \text { H } & -1.447555 & 1.720613 & -1.369100\end{array}$




$\begin{array}{rrrr}\mathrm{H} & -1.466188 & 1.810292 & 1.284317 \\ \mathrm{H} & 1.317993 & 1.783265 & -1.334866 \\ \mathrm{H} & 1.386152 & -0.755447 & -2.051376 \\ \mathrm{H} & 1.412435 & -2.223291 & 0.130820 \\ \mathrm{H} & 1.401799 & -0.581840 & 2.200072 \\ \mathrm{H} & 1.302569 & 1.894726 & 1.290950 \\ \mathrm{H} & -1.379463 & -0.822241 & -2.085922 \\ \mathrm{H} & -1.356275 & -2.284461 & 0.128885\end{array}$

\section{$\mathrm{HF}=\mathbf{- 3 8 6 . 2 4 7 5 7 8 4}$}

Thermal correction to Enthalpy $=\mathbf{0 . 1 6 4 0 4 5}$

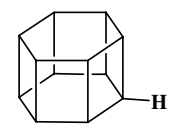

\section{Charge $=0$ Multiplicity $=1$}

$\begin{array}{lrrr}\mathrm{C} & -0.780493 & 1.351412 & -0.782720 \\ \mathrm{C} & -0.780383 & 1.351308 & 0.783136 \\ \mathrm{C} & 0.779681 & 1.350947 & 0.782940 \\ \mathrm{C} & 1.560048 & -0.000080 & 0.782759 \\ \mathrm{C} & 1.559960 & 0.000139 & -0.782960 \\ \mathrm{C} & 0.779578 & 1.351095 & -0.782773 \\ \mathrm{C} & -1.559953 & -0.000111 & 0.782939 \\ \mathrm{C} & -0.779582 & -1.351102 & 0.782771 \\ \mathrm{C} & 0.780484 & -1.351430 & 0.782731 \\ \mathrm{C} & -1.560054 & 0.000049 & -0.782723 \\ \mathrm{C} & -0.779654 & -1.350972 & -0.782954 \\ \mathrm{C} & 0.780380 & -1.351249 & -0.783128 \\ \mathrm{H} & 1.246144 & -2.158367 & 1.355762 \\ \mathrm{H} & 2.491674 & -0.000010 & 1.355874 \\ \mathrm{H} & 1.245720 & 2.157558 & 1.356120 \\ \mathrm{H} & 1.246105 & -2.157955 & -1.356440 \\ \mathrm{H} & 2.491495 & 0.000185 & -1.356218 \\ \mathrm{H} & 1.245388 & 2.157897 & -1.355875 \\ \mathrm{H} & -1.246229 & 2.158202 & -1.355908 \\ \mathrm{H} & -2.491608 & 0.000079 & -1.355942 \\ \mathrm{H} & -1.245698 & -2.157558 & -1.356174 \\ \mathrm{H} & -1.246029 & 2.157970 & 1.356563 \\ \mathrm{H} & -2.491466 & -0.000233 & 1.356254 \\ \mathrm{H} & -1.245570 & -2.157800 & 1.355874 \\ & & & \\ \text { HF }= & -\mathbf{4 6 4 . 2 9 5 0 7 4 4} & & \\ \mathrm{Th} & & & \\ & & & \end{array}$

Thermal correction to Enthalpy= 0.214192 


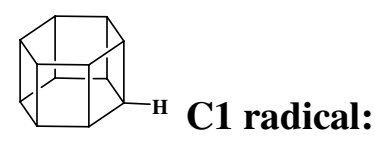

\section{Charge $=\mathbf{0}$ Multiplicity $=\mathbf{2}$}

$\begin{array}{lrrr}\text { C } & -0.800498 & 1.316310 & -0.755171 \\ \mathrm{C} & -0.796721 & 1.330779 & 0.808290 \\ \mathrm{C} & 0.765459 & 1.325840 & 0.806202 \\ \mathrm{C} & 1.551009 & -0.024473 & 0.807985 \\ \mathrm{C} & 1.540064 & -0.034803 & -0.755474 \\ \mathrm{C} & 0.758700 & 1.314485 & -0.760716 \\ \mathrm{C} & -1.573529 & -0.028722 & 0.808126 \\ \mathrm{C} & -0.758600 & -1.314306 & 0.744428 \\ \mathrm{C} & 0.762234 & -1.377067 & 0.807824 \\ \mathrm{C} & -1.585267 & -0.033359 & -0.765552 \\ \mathrm{C} & -0.799040 & -1.384016 & -0.791443 \\ \mathrm{C} & 0.763783 & -1.389372 & -0.765856 \\ \mathrm{H} & 1.210440 & -2.192174 & 1.386173 \\ \mathrm{H} & 2.486679 & -0.023721 & 1.373572 \\ \mathrm{H} & 1.233769 & 2.136974 & 1.370465 \\ \mathrm{H} & 1.236922 & -2.198990 & -1.329502 \\ \mathrm{H} & 2.469721 & -0.038108 & -1.330893 \\ \mathrm{H} & 1.221930 & 2.117075 & -1.341362 \\ \mathrm{H} & -1.268411 & 2.119766 & -1.330405 \\ \mathrm{H} & -2.523098 & -0.028483 & -1.329011 \\ \mathrm{H} & -1.266193 & -2.193146 & -1.361618 \\ \mathrm{H} & -1.263902 & 2.141342 & 1.374060 \\ \mathrm{H} & -2.503421 & -0.048308 & 1.386653 \\ & & & \\ \text { HF }= & -\mathbf{4 6 3 . 6 2 6 0 8 6 9} & & \\ \mathrm{T} & & \end{array}$

Thermal correction to Enthalpy $=0.200678$

Cyclopropene:

\section{Charge $=0$ Multiplicity $=1$}

$\begin{array}{lrrr}\mathrm{C} & 0.513853 & 0.032184 & -0.636890 \\ \mathrm{C} & 0.488601 & -0.031948 & 0.656171 \\ \mathrm{H} & 1.074157 & 0.079878 & -1.558712 \\ \mathrm{H} & 1.011105 & -0.077225 & 1.600052 \\ \mathrm{C} & -0.861413 & -0.000397 & -0.016663 \\ \mathrm{H} & -1.467319 & 0.910768 & 0.017212 \\ \mathrm{H} & -1.464188 & -0.912455 & -0.074258 \\ & & & \\ \text { HF }=\mathbf{- 1 1 6 . 6 1 9 0 3 9 6} & & \end{array}$

Thermal correction to Enthalpy $=\mathbf{0 . 0 6 0 5 4 2}$ 


\section{Cyclopropene $\mathrm{C} 1$ radical:}

\section{Charge $=0$ Multiplicity $=2$}

$\begin{array}{lrrr}\text { C } & -0.388631 & -0.009639 & -0.749825 \\ \text { C } & -0.124504 & -0.027565 & 0.829466 \\ \text { C } & 0.879437 & 0.059744 & 0.036238 \\ \text { H } & -0.815413 & 0.891001 & -1.197217 \\ \text { H } & -0.689424 & -0.938956 & -1.239066 \\ \text { H } & -0.692976 & -0.087282 & 1.741009 \\ & & & \\ \text { HF }=\mathbf{- 1 1 5 . 9 3 6 9 3 9 2} & & \end{array}$

Thermal correction to Enthalpy $=0.047695$

Cyclopropene $\mathrm{C} 3$ radical:

Charge $=0$ Multiplicity $=2$

$\begin{array}{lrrr}\mathrm{C} & 0.407302 & -0.067257 & -0.661646 \\ \mathrm{C} & 0.418380 & -0.089427 & 0.652782 \\ \mathrm{H} & 0.965308 & -0.109895 & -1.586125 \\ \mathrm{H} & 0.989470 & -0.162452 & 1.567110 \\ \mathrm{C} & -0.889710 & 0.050603 & 0.007258 \\ \mathrm{H} & -1.570612 & 0.908831 & 0.028650\end{array}$

$H F=\mathbf{- 1 1 5 . 9 5 0 7 4 1 2}$

Thermal correction to Enthalpy= 0.046946

\section{Cyclobutene:}

Charge $=0$ Multiplicity $=1$

$\begin{array}{lrrr}\text { C } & 0.793785 & 0.018042 & -0.694665 \\ \text { C } & 0.834929 & -0.020197 & 0.644616 \\ \text { H } & 1.557235 & 0.039235 & -1.468406 \\ \text { H } & 1.644660 & -0.042663 & 1.369712 \\ \text { C } & -0.675165 & -0.021816 & 0.806879 \\ \text { C } & -0.723336 & 0.023540 & -0.763878 \\ \text { H } & -1.181764 & 0.927101 & -1.184662 \\ \text { H } & -1.186180 & -0.851856 & -1.236183 \\ \text { H } & -1.105852 & 0.854830 & 1.306533 \\ \text { H } & -1.109375 & -0.924064 & 1.255293 \\ & & & \\ \text { HF }=\mathbf{- 1 5 5 . 9 7 3 2 6 4 3} & & \end{array}$

Thermal correction to Enthalpy $=0.091691$

Cyclobutene $\mathrm{C} 1$ radical:

Charge $=0$ Multiplicity $=2$
C $\quad 0.900608$
$0.012259-0.636435$
$\begin{array}{llll}\text { C } & 0.840235 & -0.030134 & 0.693237\end{array}$ 


$\begin{array}{rrrr}\text { H } & 1.679393 & 0.032359 & -1.388891 \\ \mathrm{C} & -0.667175 & -0.023064 & 0.851440 \\ \mathrm{C} & -0.629277 & 0.030515 & -0.721969 \\ \mathrm{H} & -1.059673 & 0.948737 & -1.140022 \\ \mathrm{H} & -1.078824 & -0.847011 & -1.202643 \\ \mathrm{H} & -1.096978 & 0.857834 & 1.338169 \\ \mathrm{H} & -1.110267 & -0.929373 & 1.275747 \\ & & & \\ \mathbf{H F}=\mathbf{- 1 5 5 . 2 8 7 2 2 5 8} & & \end{array}$

Thermal correction to Enthalpy $=\mathbf{0 . 0 7 9 0 5 8}$

Cyclobutene $\mathrm{C3}$ radical:

Charge $=0$ Multiplicity $=2$

$\begin{array}{lrrr}\text { C } & 0.741794 & 0.192678 & -0.677891 \\ \mathrm{C} & 0.778515 & -0.059661 & 0.696730 \\ \mathrm{H} & 1.483837 & 0.401865 & -1.439430 \\ \mathrm{H} & 1.586296 & -0.121133 & 1.420646 \\ \mathrm{C} & -0.608653 & -0.203643 & 0.796884 \\ \mathrm{C} & -0.785662 & 0.060373 & -0.703227 \\ \mathrm{H} & -1.339905 & 0.971106 & -0.965854 \\ \mathrm{H} & -1.184546 & -0.775318 & -1.293076 \\ \mathrm{H} & -1.301646 & -0.415006 & 1.602736 \\ & & & \\ \text { HF= -155.3199749 } & & \end{array}$

Thermal correction to Enthalpy $=\mathbf{0 . 0 7 7 5 8 0}$

\section{Cyclobutadiene:}

\section{Charge $=0$ Multiplicity $=1$}

$\begin{array}{lccc}\text { C } & 0.789143 & 0.000000 & -0.667284 \\ \mathrm{C} & 0.789143 & 0.000000 & 0.667284 \\ \mathrm{H} & 1.550193 & 0.000000 & -1.439120 \\ \mathrm{H} & 1.550193 & 0.000000 & 1.439120 \\ \mathrm{C} & -0.789143 & 0.000000 & 0.667284 \\ \mathrm{C} & -0.789143 & 0.000000 & -0.667284 \\ \mathrm{H} & -1.550193 & 0.000000 & 1.439120 \\ \mathrm{H} & -1.550193 & 0.000000 & -1.439120 \\ & & & \\ \text { HF= -154.6754629 } & & \end{array}$

Thermal correction to Enthalpy= 0.065942

Cyclobutadiene $\mathrm{C} 1$ radical:

Charge $=0$ Multiplicity $=2$
C $\quad-0.411852$
0.862684
$-0.472310$
$\begin{array}{llll}\text { C } & -0.630204 & -0.535789 & -0.269339\end{array}$ 


$\begin{array}{lrrr}\text { C } & 0.324814 & -0.638188 & 0.789913 \\ \text { C } & 0.867011 & 0.494320 & 0.082006 \\ \text { H } & -0.773800 & 1.618601 & -1.154472 \\ \text { H } & -0.847289 & -1.287568 & -1.036683 \\ \text { H } & 0.722476 & -1.429195 & 1.409534 \\ & \\ \text { HF }= & -\mathbf{- 1 5 4 . 0 0 8 2 5 3 9} \\ \text { Thermal correction to Enthalpy }=\mathbf{0 . 0 5 2 3 9 1}\end{array}$

\section{Cyclopentene:}

Charge $=0$ Multiplicity $=1$

$\begin{array}{lrrr}\mathrm{C} & -0.975785 & -0.146993 & -0.741989 \\ \mathrm{C} & -0.982453 & -0.134902 & 0.810849 \\ \mathrm{C} & 0.489519 & -0.153323 & 1.158666 \\ \mathrm{C} & 1.259949 & 0.094178 & 0.096429 \\ \mathrm{C} & 0.444724 & 0.323675 & -1.156866 \\ \mathrm{H} & 2.344290 & 0.167219 & 0.113903 \\ \mathrm{H} & 0.852391 & -0.312069 & 2.170870 \\ \mathrm{H} & 0.829639 & -0.225320 & -2.025841 \\ \mathrm{H} & 0.458280 & 1.388523 & -1.436469 \\ \mathrm{H} & -1.462365 & 0.771386 & 1.211581 \\ \mathrm{H} & -1.533961 & -0.984795 & 1.232982 \\ \mathrm{H} & -1.134676 & -1.171810 & -1.096062 \\ \mathrm{H} & -1.769321 & 0.471051 & -1.173502 \\ & & & \\ \text { HF= -195.3271388 } & & \\ \text { Thr }\end{array}$

Thermal correction to Enthalpy $=\mathbf{0 . 1 2 2 8 9 0}$

Cyclopentene $\mathrm{C} 1$ radical:

Charge $=0$ Multiplicity $=2$

$\begin{array}{rrrr}\text { C } & -0.909893 & -0.135198 & -0.739088 \\ \text { C } & -0.915264 & -0.138769 & 0.820568 \\ \text { C } & 0.567342 & -0.146279 & 1.183149 \\ \text { C } & 1.278629 & 0.100213 & 0.095163 \\ \text { C } & 0.520692 & 0.328513 & -1.174946 \\ \text { H } & 0.935980 & -0.294699 & 2.193084 \\ \text { H } & 0.903268 & -0.245117 & -2.026657 \\ \text { H } & 0.544865 & 1.388041 & -1.465389 \\ \text { H } & -1.403976 & 0.757945 & 1.229076 \\ \text { H } & -1.455189 & -1.003454 & 1.226644 \\ \text { H } & -1.078610 & -1.153211 & -1.105043 \\ \text { H } & -1.695375 & 0.499614 & -1.160795 \\ & & & \\ \text { HF }=\mathbf{- 1 9 4 . 6 3 7 9 8 6} & & \end{array}$


Thermal correction to Enthalpy= $\mathbf{0 . 1 1 0 2 0 0}$

Cyclopentene $\mathrm{C} 3$ radical:

Charge $=\mathbf{0}$ Multiplicity $=2$

$\begin{array}{lrrr}\text { C } & -0.966924 & -0.021904 & -0.786375 \\ \text { C } & -0.946968 & -0.315125 & 0.746949 \\ \text { C } & 0.498890 & -0.129727 & 1.136519 \\ \text { C } & 1.256991 & 0.221204 & 0.025847 \\ \text { C } & 0.469712 & 0.300400 & -1.116515 \\ \text { H } & 2.326713 & 0.409725 & 0.047961 \\ \text { H } & 0.873418 & -0.257511 & 2.146264 \\ \text { H } & 0.817808 & 0.555881 & -2.111364 \\ \text { H } & -1.609852 & 0.364760 & 1.301639 \\ \text { H } & -1.303733 & -1.330429 & 0.973350 \\ \text { H } & -1.334844 & -0.883490 & -1.362256 \\ \text { H } & -1.639721 & 0.811971 & -1.034151 \\ & & & \\ \text { HF = - 194.6860804 } & & \end{array}$

Thermal correction to Enthalpy= 0.108710

Cyclopentene $\mathrm{C} 4$ radical:

Charge $=\mathbf{0}$ Multiplicity $=2$

$\begin{array}{rrrr}\text { C } & -0.994058 & 0.107386 & -0.695969 \\ \mathrm{C} & -1.016131 & -0.197165 & 0.774627 \\ \mathrm{C} & 0.458430 & -0.214976 & 1.122230 \\ \mathrm{C} & 1.229297 & 0.032730 & 0.059414 \\ \mathrm{C} & 0.413552 & 0.262218 & -1.196520 \\ \mathrm{H} & 2.314976 & 0.070983 & 0.066871 \\ \mathrm{H} & 0.819983 & -0.409406 & 2.128060 \\ \mathrm{H} & 0.669721 & -0.458759 & -1.994290 \\ \mathrm{H} & 0.607179 & 1.256061 & -1.640020 \\ \mathrm{H} & -1.571089 & 0.556145 & 1.363231 \\ \mathrm{H} & -1.508542 & -1.158672 & 1.008942 \\ \mathrm{H} & -1.878769 & 0.202486 & -1.315490 \\ & & & \\ \text { HF }=\mathbf{- 1 9 4 . 6 6 3 8 0 6 7} & & \end{array}$

Thermal correction to Enthalpy= $\mathbf{0 . 1 0 7 7 9 7}$

\section{Cyclopentadiene:}

\section{Charge $=\mathbf{0}$ Multiplicity $=1$}
C $\quad 0.592206$
0.750597
0.748179
C $\quad 0.592140$
$-0.756025$
0.748225
C $\quad-0.318065 \quad-1.100545$
$-0.401881$ 


$\begin{array}{lrrr}\text { C } & -0.765323 & 0.039830 & -0.966956 \\ \text { C } & -0.199051 & 1.191403 & -0.251542 \\ \text { H } & -0.556734 & -2.113930 & -0.703427 \\ \text { H } & -1.437264 & 0.115030 & -1.815908 \\ \text { H } & -0.398134 & 2.228207 & -0.503096 \\ \text { H } & 1.149898 & 1.356705 & 1.452777 \\ \text { H } & 0.226950 & -1.168731 & 1.701566 \\ \text { H } & 1.603843 & -1.168845 & 0.611935\end{array}$

\section{$H F=\mathbf{- 1 9 4 . 1 0 1 0 5 7 3 ~}$}

Thermal correction to Enthalpy= 0.097991

\section{Cyclopentadiene $\mathrm{C} 1$ radical:}

\section{Charge $=\mathbf{0}$ Multiplicity $=2$}

$\begin{array}{lrrr}\mathrm{C} & -0.958994 & 0.000905 & -0.789832 \\ \mathrm{C} & -1.040072 & -0.000406 & 0.704897 \\ \mathrm{C} & 0.433363 & -0.000803 & 1.071535 \\ \mathrm{C} & 1.175990 & -0.000157 & -0.054935 \\ \mathrm{C} & 0.296404 & 0.000857 & -1.248517 \\ \mathrm{H} & -1.568734 & 0.879958 & 1.096610 \\ \mathrm{H} & -1.567888 & -0.882312 & 1.094411 \\ \mathrm{H} & 0.795203 & -0.000893 & 2.093461 \\ \mathrm{H} & 2.260868 & -0.000085 & -0.107405 \\ \mathrm{H} & 0.640401 & 0.000954 & -2.275962\end{array}$

$\mathrm{HF}=\mathbf{- 1 9 3 . 4 0 7 6 9 3}$

Thermal correction to Enthalpy= $\mathbf{0 . 0 8 5 2 9 9}$

\section{Cyclopentadiene $\mathrm{C} 2$ radical:}

\section{Charge $=0$ Multiplicity $=\mathbf{2}$}

$\begin{array}{lrrr}\text { C } & 1.096900 & 0.000684 & -0.633814 \\ \mathrm{C} & 1.045789 & 0.000937 & 0.701267 \\ \mathrm{H} & 1.957913 & 0.000982 & -1.289802 \\ \mathrm{C} & -0.311305 & 0.000097 & 1.236893 \\ \mathrm{C} & -1.149514 & -0.000677 & 0.176567 \\ \mathrm{H} & -0.577583 & 0.000100 & 2.286993 \\ \mathrm{H} & -2.233961 & -0.001017 & 0.205899 \\ \mathrm{C} & -0.348376 & -0.000583 & -1.105911 \\ \mathrm{H} & -0.574496 & 0.879349 & -1.727373 \\ \mathrm{H} & -0.572832 & -0.882168 & -1.725730 \\ & & & \\ \text { HF }=\mathbf{- 1 9 3 . 4 0 8 8 4 4 4} & & \end{array}$

Thermal correction to Enthalpy= $\mathbf{0 . 0 8 5 1 0 9}$ 


\section{Cyclopentadiene $\mathrm{C} 5$ radical:}

\section{Charge $=\mathbf{0}$ Multiplicity $=2$}

$\begin{array}{lrrr}\text { C } & 0.977525 & 0.049946 & -0.734182 \\ \mathrm{C} & 1.020097 & -0.124752 & 0.625229 \\ \mathrm{H} & 1.818699 & 0.099001 & -1.413580 \\ \mathrm{H} & 1.905195 & -0.241298 & 1.240211 \\ \mathrm{C} & -0.371185 & -0.128474 & 1.137632 \\ \mathrm{C} & -1.220081 & 0.044611 & 0.076043 \\ \mathrm{H} & -0.645340 & -0.248048 & 2.179574 \\ \mathrm{H} & -2.301312 & 0.087427 & 0.103349 \\ \mathrm{C} & -0.406153 & 0.158655 & -1.104107 \\ \mathrm{H} & -0.778457 & 0.303010 & -2.113248 \\ & & & \\ \text { HF }=\mathbf{- 1 9 3 . 4 6 2 3 2 1} & & \end{array}$

Thermal correction to Enthalpy $=0.084029$

\section{Benzene:}

\begin{tabular}{cccc}
\multicolumn{5}{c}{ Charge $=\mathbf{0}$ Multiplicity $=\mathbf{1}$} \\
$\mathrm{C}$ & -1.209110 & 0.000000 & -0.698080 \\
$\mathrm{C}$ & -1.209110 & 0.000000 & 0.698080 \\
$\mathrm{C}$ & 0.000000 & 0.000000 & 1.396160 \\
$\mathrm{C}$ & 1.209110 & 0.000000 & 0.698080 \\
$\mathrm{C}$ & 1.209110 & 0.000000 & -0.698080 \\
$\mathrm{C}$ & 0.000000 & 0.000000 & -1.396160 \\
$\mathrm{H}$ & -2.150353 & 0.000000 & 1.241507 \\
$\mathrm{H}$ & 0.000000 & 0.000000 & 2.483014 \\
$\mathrm{H}$ & 2.150353 & 0.000000 & 1.241507 \\
$\mathrm{H}$ & 2.150353 & 0.000000 & -1.241507 \\
$\mathrm{H}$ & 0.000000 & 0.000000 & -2.483014 \\
$\mathrm{H}$ & $\mathbf{- 2 . 1 5 0 3 5 3}$ & 0.000000 & -1.241507 \\
& & & \\
HF $=\mathbf{- 2 3 2 . 2 4 8 6 5 8 8}$ & &
\end{tabular}

Thermal correction to Enthalpy $=0.106108$

Benzene $\mathrm{C1}$ radical:

Charge $=0$ Multiplicity $=2$

$\begin{array}{lrrr}\mathrm{C} & -1.212587 & 0.000000 & -0.700088 \\ \mathrm{C} & -1.281579 & 0.000000 & 0.676180 \\ \mathrm{C} & -0.059243 & 0.000000 & 1.367821 \\ \mathrm{C} & 1.147252 & 0.000000 & 0.662366 \\ \mathrm{C} & 1.154946 & 0.000000 & -0.735216 \\ \mathrm{C} & -0.055200 & 0.000000 & -1.447970 \\ \mathrm{H} & -2.227454 & 0.000000 & 1.211472 \\ \mathrm{H} & -0.056213 & 0.000000 & 2.455540\end{array}$




$\begin{array}{rrrr}\text { H } & 2.088243 & 0.000000 & 1.205647 \\ \mathrm{H} & 2.098454 & 0.000000 & -1.276452 \\ \mathrm{H} & -0.064561 & 0.000000 & -2.534768 \\ & & & \\ \mathbf{H F}=\mathbf{- 2 3 1 . 5 6 1 2 8 0 8} & & \end{array}$

Thermal correction to Enthalpy $=0.092938$

Bicyclo[2.1.0]pent-2-ene:

Charge $=0$ Multiplicity $=1$

$\begin{array}{lrrr}\mathrm{C} & -0.628895 & -0.538349 & -0.421310 \\ \mathrm{C} & -0.635341 & -0.527965 & 1.098629 \\ \mathrm{C} & 0.685470 & -0.522463 & 0.346324 \\ \mathrm{C} & 0.921619 & 0.851822 & -0.262686 \\ \mathrm{C} & -0.238961 & 0.837671 & -0.940866 \\ \mathrm{H} & -0.796276 & 1.588450 & -1.494661 \\ \mathrm{H} & -1.023618 & -1.369648 & -0.996373 \\ \mathrm{H} & 1.395113 & -1.340413 & 0.416128 \\ \mathrm{H} & -0.906249 & -1.467581 & 1.581889 \\ \mathrm{H} & -0.956324 & 0.366413 & 1.629826 \\ \mathrm{H} & 1.664000 & 1.618482 & -0.057359\end{array}$

$\mathrm{HF}=\mathbf{- 1 9 4 . 0 2 4 3 7 4 7}$

Thermal correction to Enthalpy= 0.097254

Bicyclo[2.1.0]pent-2-ene $\mathrm{C} 1$ radical:

Charge $=0$ Multiplicity $=2$

\begin{tabular}{lrrr} 
C & -0.650269 & 0.392207 & -0.990691 \\
$\mathrm{C}$ & -0.626714 & 0.421181 & 0.536042 \\
$\mathrm{C}$ & 0.828329 & 0.407143 & 1.065158 \\
$\mathrm{C}$ & 0.231300 & -0.799702 & 0.415800 \\
$\mathrm{C}$ & 0.100607 & -0.727471 & -1.077717 \\
$\mathrm{H}$ & -0.966362 & 1.131944 & -1.724602 \\
$\mathrm{H}$ & -1.468434 & 0.575025 & 1.203544 \\
$\mathrm{H}$ & 0.907519 & 0.388938 & 2.152357 \\
$\mathrm{H}$ & 1.610203 & 0.978562 & 0.562954 \\
$\mathrm{H}$ & 0.617554 & -1.234616 & -1.885809 \\
\multicolumn{4}{l}{} \\
HF= -193.3392615 &
\end{tabular}

Bicyclo[2.1.0]pent-2-ene C2 radical:

Charge $=0$ Multiplicity $=2$
C $\quad-0.603062$
$0.461041-1.086953$
$\begin{array}{llll}\text { C } & -0.591152 & 0.436708 & 0.430939\end{array}$ 


$\begin{array}{rrrr}\text { C } & 0.828918 & 0.432315 & 0.953645 \\ \text { C } & 0.269298 & -0.849404 & 0.369541 \\ \text { C } & 0.045010 & -0.715415 & -1.112758 \\ \text { H } & -0.877298 & 1.219098 & -1.809601 \\ \text { H } & -1.464161 & 0.502139 & 1.075323 \\ \text { H } & 0.105669 & -1.736465 & 0.970902 \\ \text { H } & 0.953998 & 0.442525 & 2.036790 \\ \text { H } & 1.587721 & 0.981240 & 0.400106 \\ & & & \\ \text { HF }=\mathbf{- 1 9 3 . 3 4 2 4 9 5 9} & & \end{array}$

Thermal correction to Enthalpy= $\mathbf{0 . 0 8 4 6 0 8}$

Bicyclo[2.1.0]pent-2-ene C5 radical:

Charge $=0$ Multiplicity $=2$

$\begin{array}{lrrr}\mathrm{C} & -0.585535 & 0.486376 & -1.010580 \\ \mathrm{C} & -0.566467 & 0.547972 & 0.509679 \\ \mathrm{C} & 0.796050 & 0.453583 & 1.073174 \\ \mathrm{C} & 0.315955 & -0.779633 & 0.416243 \\ \mathrm{C} & 0.153699 & -0.625662 & -1.088847 \\ \mathrm{H} & -0.949000 & 1.204816 & -1.739185 \\ \mathrm{H} & -1.448806 & 0.679637 & 1.131945 \\ \mathrm{H} & 0.133703 & -1.701274 & 0.964364 \\ \mathrm{H} & 0.956588 & 0.484441 & 2.150722 \\ \mathrm{H} & 0.625303 & -1.163441 & -1.905862\end{array}$

$\mathrm{HF}=\mathbf{- 1 9 3 . 3 4 6 5 3 4 2}$

Thermal correction to Enthalpy $=\mathbf{0 . 0 8 3 4 9 3}$

Bicyclo[2.1.1]hex-2-ene:

Charge $=0$ Multiplicity $=1$

$\begin{array}{lrrr}\mathrm{C} & -0.608787 & 0.674783 & -0.982239 \\ \mathrm{C} & -0.572176 & 0.672662 & 0.586002 \\ \mathrm{C} & 0.944035 & 0.594448 & 0.826763 \\ \mathrm{C} & 1.348332 & -0.326851 & -0.057248 \\ \mathrm{C} & 0.044896 & -0.734214 & -0.763001 \\ \mathrm{C} & -0.917862 & -0.852995 & 0.469766 \\ \mathrm{H} & -1.165711 & 1.364594 & 1.187706 \\ \mathrm{H} & 0.086578 & -1.490566 & -1.549955 \\ \mathrm{H} & -1.961543 & -1.044559 & 0.192162 \\ \mathrm{H} & -0.607026 & -1.505063 & 1.292008 \\ \mathrm{H} & -0.012711 & 1.432718 & -1.500044 \\ \mathrm{H} & -1.623560 & 0.626055 & -1.395630 \\ \mathrm{H} & 1.513696 & 1.166504 & 1.549549 \\ \mathrm{H} & 2.339642 & -0.716672 & -0.256055\end{array}$


$\mathrm{HF}=\mathbf{- 2 3 3 . 3 6 5 0 0 8}$

Thermal correction to Enthalpy= $\mathbf{0 . 1 2 8 5 4 2}$

Bicyclo[2.1.1]hex-2-ene C1 radical:

Charge $=\mathbf{0}$ Multiplicity $=\mathbf{2}$

$\begin{array}{lrrr}\mathrm{C} & -0.655934 & -0.689074 & -0.978467 \\ \mathrm{C} & -0.626582 & -0.641472 & 0.568932 \\ \mathrm{C} & 0.866346 & -0.676966 & 0.880583 \\ \mathrm{C} & 1.339558 & 0.223337 & 0.005871 \\ \mathrm{C} & 0.067257 & 0.690453 & -0.723279 \\ \mathrm{C} & -0.924523 & 0.875881 & 0.490626 \\ \mathrm{H} & 0.145769 & 1.445826 & -1.513518 \\ \mathrm{H} & -1.652640 & -0.578379 & -1.419590 \\ \mathrm{H} & -0.080456 & -1.473580 & -1.475486 \\ \mathrm{H} & -0.591472 & 1.504418 & 1.319884 \\ \mathrm{H} & -1.945102 & 1.124937 & 0.179718 \\ \mathrm{H} & 1.374824 & -1.283128 & 1.619933 \\ \mathrm{H} & 2.352346 & 0.566955 & -0.176531 \\ & & & \\ \text { HF }=\mathbf{- 2 3 2 . 6 8 3 7 1 4 3} & & \\ \text { The }\end{array}$

Thermal correction to Enthalpy $=\mathbf{0 . 1 1 5 4 1 5}$

Bicyclo[2.1.1]hex-2-ene $\mathrm{C} 2$ radical:

Charge $=0$ Multiplicity $=2$

$\begin{array}{lrrr}\text { C } & -0.585038 & -0.676211 & -0.960478 \\ \mathrm{C} & -0.601040 & -0.677042 & 0.611692 \\ \mathrm{C} & 0.907010 & -0.653633 & 0.861934 \\ \mathrm{C} & 1.425638 & 0.228520 & 0.017138 \\ \mathrm{C} & 0.129620 & 0.697869 & -0.720007 \\ \mathrm{C} & -0.850147 & 0.870686 & 0.491087 \\ \mathrm{H} & -1.236529 & -1.339044 & 1.201097 \\ \mathrm{H} & 0.235772 & 1.452129 & -1.504403 \\ \mathrm{H} & -1.584756 & -0.582874 & -1.402723 \\ \mathrm{H} & -0.010525 & -1.463829 & -1.456198 \\ \mathrm{H} & -0.518145 & 1.498045 & 1.323158 \\ \mathrm{H} & -1.876822 & 1.121468 & 0.196512 \\ \mathrm{H} & 2.434736 & 0.572977 & -0.165638 \\ & & & \\ \text { HF }=\mathbf{- 2 3 2 . 6 7 3 3 7 8} & & \end{array}$

Thermal correction to Enthalpy= 0.115877 


\section{Bicyclo[2.1.1]hex-2-ene C5 radical:}

\section{Charge $=0$ Multiplicity $=2$}

$\begin{array}{lrrr}\text { C } & -0.687228 & -0.602101 & -0.992053 \\ \text { C } & -0.670636 & -0.620568 & 0.574293 \\ \text { C } & 0.847033 & -0.626384 & 0.844022 \\ \text { C } & 1.320663 & 0.277441 & -0.032718 \\ \text { C } & 0.052668 & 0.759826 & -0.764564 \\ \mathrm{C} & -0.858402 & 0.905497 & 0.469855 \\ \text { H } & -1.311617 & -1.274965 & 1.167716 \\ \text { H } & 0.152415 & 1.519105 & -1.542245 \\ \text { H } & -1.693855 & -0.475732 & -1.405593 \\ \text { H } & -0.136923 & -1.394479 & -1.511713 \\ \text { H } & -0.739647 & 1.629127 & 1.280084 \\ \text { H } & 1.371703 & -1.200196 & 1.599412 \\ \text { H } & 2.333336 & 0.634871 & -0.180672 \\ & & & \\ \text { HF = -232.6904506 } & & \end{array}$

Thermal correction to Enthalpy $=\mathbf{0 . 1 1 4 7 9 8}$

Tricyclo[2.1.1.01,4]hex-2-ene:

Charge $=0$ Multiplicity $=1$

$\begin{array}{lrrr}\text { C } & -0.492829 & 0.653005 & -1.179264 \\ \mathrm{C} & -0.469524 & 0.645800 & 0.335896 \\ \mathrm{C} & 0.975406 & 0.566679 & 0.833583 \\ \mathrm{C} & 1.305098 & -0.501586 & 0.104314 \\ \mathrm{C} & -0.065794 & -0.661863 & -0.556818 \\ \mathrm{C} & -1.146737 & -0.699315 & 0.505494 \\ \mathrm{H} & -2.176950 & -0.779481 & 0.156627 \\ \mathrm{H} & -0.937884 & -1.255470 & 1.415805 \\ \mathrm{H} & 0.267486 & 1.237207 & -1.691293 \\ \mathrm{H} & -1.473434 & 0.675738 & -1.656132 \\ \mathrm{H} & 2.200529 & -1.101475 & -0.030756 \\ \mathrm{H} & 1.486534 & 1.207160 & 1.546526 \\ & & & \\ \text { HF }=\mathbf{- 2 3 2 . 0 7 7 3 5 6 3} & & \end{array}$

Thermal correction to Enthalpy= $\mathbf{0 . 1 0 3 9 2 5}$

Tricyclo[2.1.1.01,4]hex-2-ene $\mathrm{C} 2$ radical:

Charge $=0$ Multiplicity $=2$

$\begin{array}{lrrr}\text { C } & -0.451924 & -0.591150 & -1.200559 \\ \text { C } & -0.422937 & -0.577233 & 0.320787 \\ \text { C } & 1.003387 & -0.620037 & 0.871152 \\ \text { C } & 1.342761 & 0.434690 & 0.128288 \\ \text { C } & 0.023271 & 0.698311 & -0.564108\end{array}$




$\begin{array}{lrrr}\mathrm{C} & -1.062271 & 0.793754 & 0.487319 \\ \mathrm{H} & -1.432939 & -0.581183 & -1.676379 \\ \mathrm{H} & 0.292195 & -1.203763 & -1.701414 \\ \mathrm{H} & -0.828204 & 1.338333 & 1.397566 \\ \mathrm{H} & -2.088856 & 0.905814 & 0.137426 \\ \mathrm{H} & 1.464086 & -1.289209 & 1.585528\end{array}$

$\mathrm{HF}=\mathbf{- 2 3 1 . 3 9 7 8 6 3}$

Thermal correction to Enthalpy $=0.091528$

Tricyclo[2.1.1.01,4]hex-2-ene $\mathrm{C5}$ radical:

Charge $=0$ Multiplicity $=2$

$\begin{array}{rrrr}\text { C } & -0.514445 & -0.628977 & -1.204825 \\ \mathrm{C} & -0.549367 & -0.642036 & 0.274666 \\ \mathrm{C} & 0.891784 & -0.635822 & 0.808973 \\ \mathrm{C} & 1.291664 & 0.422125 & 0.085942 \\ \mathrm{C} & -0.062263 & 0.646844 & -0.606182 \\ \mathrm{C} & -1.154859 & 0.740292 & 0.444670 \\ \mathrm{H} & 0.102249 & -1.280076 & -1.816496 \\ \mathrm{H} & -0.921930 & 1.278333 & 1.360773 \\ \mathrm{H} & -2.170625 & 0.874235 & 0.078912 \\ \mathrm{H} & 2.215081 & 0.987385 & 0.005773 \\ \mathrm{H} & 1.360141 & -1.274431 & 1.551575 \\ & & & \\ \mathbf{H F}=\mathbf{- 2 3 1 . 4 0 3 2 9 2 3} & & \end{array}$

Thermal correction to Enthalpy= $\mathbf{0 . 0 9 0 5 3 1}$

Bicyclo[2.2.0]hexa-2,5-diene:

Charge $=0$ Multiplicity $=1$

$\begin{array}{lrrr}\text { C } & -1.241937 & 0.483119 & -0.675440 \\ \mathrm{C} & -1.249746 & 0.477346 & 0.664603 \\ \mathrm{C} & 0.274784 & 0.435824 & 0.790135 \\ \mathrm{C} & 0.958556 & -0.927947 & 0.671887 \\ \mathrm{C} & 0.966772 & -0.922513 & -0.667851 \\ \mathrm{C} & 0.283894 & 0.442230 & -0.783371 \\ \mathrm{H} & -2.022168 & 0.459939 & -1.431452 \\ \mathrm{H} & 1.279254 & -1.641669 & -1.420247 \\ \mathrm{H} & 0.790835 & 1.234818 & -1.341460 \\ \mathrm{H} & 0.774757 & 1.224306 & 1.360242 \\ \mathrm{H} & 1.262193 & -1.653130 & 1.421945 \\ \mathrm{H} & -2.038808 & 0.447383 & 1.411194 \\ & & & \\ \text { HF }=\mathbf{- 2 3 2 . 1 1 4 2 3 9 7} & & \end{array}$

Thermal correction to Enthalpy= $\mathbf{0 . 1 0 3 2 5 1}$ 
Bicyclo[2.2.0]hexa-2,5-diene $\mathrm{C} 1$ radical:

Charge $=0$ Multiplicity $=2$

$\begin{array}{lrrr}\mathrm{C} & -0.748033 & 0.454724 & -0.330536 \\ \mathrm{C} & -0.729979 & 0.512297 & 1.205468 \\ \mathrm{C} & 0.626864 & 0.513343 & 1.290807 \\ \mathrm{C} & 0.784324 & 0.383595 & -0.170296 \\ \mathrm{C} & 0.800920 & -0.850198 & -0.978953 \\ \mathrm{C} & -0.553895 & -0.867136 & -1.090755 \\ \mathrm{H} & -1.521159 & 0.500317 & 1.951300 \\ \mathrm{H} & -1.255381 & -1.581756 & -1.514559 \\ \mathrm{H} & -1.215920 & 1.285215 & -0.865321 \\ \mathrm{H} & 1.584663 & -1.552617 & -1.245579 \\ \mathrm{H} & 1.326594 & 0.469091 & 2.119754\end{array}$

$\mathrm{HF}=\mathbf{- 2 3 1 . 4 4 4 8 5 3}$

Thermal correction to Enthalpy $=\mathbf{0 . 0 9 0 2 2 2}$

Bicyclo[2.2.0]hexa-2,5-diene C2 radical:

Charge $=0$ Multiplicity $=2$

$\begin{array}{lrrr}\mathrm{C} & -0.724471 & 0.449624 & -0.255899 \\ \mathrm{C} & -0.761459 & 0.448877 & 1.276222 \\ \mathrm{C} & 0.570972 & 0.488829 & 1.323701 \\ \mathrm{C} & 0.861835 & 0.450016 & -0.171400 \\ \mathrm{C} & 0.782928 & -0.895043 & -0.885747 \\ \mathrm{C} & -0.553268 & -0.894070 & -0.970260 \\ \mathrm{H} & -1.569367 & 0.382444 & 1.993321 \\ \mathrm{H} & -1.286036 & -1.604735 & -1.341680 \\ \mathrm{H} & -1.214474 & 1.278713 & -0.775774 \\ \mathrm{H} & 1.453936 & 1.256641 & -0.608356 \\ \mathrm{H} & 1.556723 & -1.602461 & -1.167215\end{array}$

$\mathrm{HF}=\mathbf{- 2 3 1 . 4 2 9 2 5 8 8}$

Thermal correction to Enthalpy $=0.090627$

Bicyclo[2.2.0]hexa-1(4),2,5-triene:

Charge $=0$ Multiplicity $=1$

$\begin{array}{lrrr}\text { C } & -0.784796 & 0.012979 & -0.042256 \\ \text { C } & -0.776978 & 0.737239 & 1.146600 \\ \text { C } & 0.673673 & 0.714736 & 1.223701 \\ \text { C } & 0.784001 & -0.011210 & 0.041115 \\ \text { C } & 0.777331 & -0.738130 & -1.145974 \\ \text { C } & -0.673381 & -0.714753 & -1.223730 \\ \text { H } & -1.552428 & 1.160865 & 1.783925\end{array}$




$\begin{array}{crrr}\mathrm{H} & -1.389637 & -1.117278 & -1.939208 \\ \mathrm{H} & 1.552819 & -1.165828 & -1.780436 \\ \mathrm{H} & 1.390151 & 1.117076 & 1.938989\end{array}$

$\mathrm{HF}=\mathbf{- 2 3 0 . 8 1 8 4 3 1 7}$

Thermal correction to Enthalpy= $\mathbf{0 . 0 7 8 4 7 4}$

Bicyclo[2.2.0]hexa-1(4),2,5-triene $\mathrm{C} 2$ radical: Charge $=0$ Multiplicity $=2$

$\begin{array}{lrrr}\mathrm{C} & -0.791591 & -0.011943 & -1.375347 \\ \mathrm{C} & -0.922682 & -0.014754 & -0.010804 \\ \mathrm{C} & -0.701873 & -0.011859 & 1.354888 \\ \mathrm{C} & 0.724307 & 0.011124 & 1.279002 \\ \mathrm{C} & 0.798495 & 0.012730 & -0.104786 \\ \mathrm{C} & 0.651255 & 0.010943 & -1.464841 \\ \mathrm{H} & -1.393301 & -0.023150 & 2.195931 \\ \mathrm{H} & 1.340624 & 0.022452 & -2.303876 \\ \mathrm{H} & 1.505215 & 0.023252 & 2.039269\end{array}$

$\mathrm{HF}=\mathbf{- 2 3 0 . 1 4 6 9 3 2 1}$

Thermal correction to Enthalpy= $\mathbf{0 . 0 6 5 4 1 6}$

Ammonia:

Charge $=0$ Multiplicity $=1$

$\begin{array}{lrrr}\mathrm{N} & -0.056473 & -0.097625 & -0.039817 \\ \mathrm{H} & -0.024507 & -0.043225 & 0.978104 \\ \mathrm{H} & 0.913800 & -0.043332 & -0.349609 \\ \mathrm{H} & -0.493979 & 0.769932 & -0.349779\end{array}$

$\mathrm{HF}=\mathbf{- 5 6 . 5 4 7 9 4 7}$

Thermal correction to Enthalpy= $\mathbf{0 . 0 3 8 3 0 9}$

\section{Ammonia $\mathrm{N}$ radical:}

\section{Charge $=\mathbf{0}$ Multiplicity $=2$}

$\begin{array}{cccc}\mathrm{N} & -0.117881 & 0.000000 & -0.083355 \\ \mathrm{H} & -0.051880 & 0.000000 & 0.948593 \\ \mathrm{H} & 0.877050 & 0.000000 & -0.365108\end{array}$

$\mathrm{HF}=\mathbf{- 5 5 . 8 7 2 6 1 8 6}$

Thermal correction to Enthalpy $=\mathbf{0 . 0 2 2 7 6 7}$

Methylamine:

Charge $=0$ Multiplicit $y=1$

$\begin{array}{llll}\text { C } & 0.307219 & -0.585595 & 0.246696\end{array}$ 


$\begin{array}{cccc}\mathrm{H} & 0.297242 & -0.608437 & 1.342240 \\ \mathrm{H} & 1.359553 & -0.660308 & -0.080821 \\ \mathrm{H} & -0.213860 & -1.484611 & -0.100502 \\ \mathrm{~N} & -0.420110 & 0.599005 & -0.215055 \\ \mathrm{H} & 0.062036 & 1.440047 & 0.097189 \\ \mathrm{H} & -0.407517 & 0.633842 & -1.232899 \\ & & & \\ \mathbf{H F}=\mathbf{- 9 5 . 8 5 3 2 0 4 5} & & \end{array}$

Thermal correction to Enthalpy $=\mathbf{0 . 0 6 8 7 5 3}$

Methylamine $\mathrm{N}$ radical:

Charge $=0$ Multiplicity $=2$

$\begin{array}{lrrr}\mathrm{C} & 0.260157 & -0.542682 & 0.182821 \\ \mathrm{H} & 0.295976 & -0.568664 & 1.286378 \\ \mathrm{H} & 1.311360 & -0.564390 & -0.155059 \\ \mathrm{H} & -0.235539 & -1.452462 & -0.169871 \\ \mathrm{~N} & -0.430940 & 0.628698 & -0.304321 \\ \mathrm{H} & 0.083840 & 1.440720 & 0.071874\end{array}$

HF $=\mathbf{- 9 5 . 1 9 0 8 6 0 4}$

Thermal correction to Enthalpy $=\mathbf{0 . 0 5 3 4 7 3}$

Dimethylamine:

Charge $=0$ Multiplicity $=1$

$\begin{array}{lrrr}\mathrm{C} & 0.454129 & -0.760004 & 0.859306 \\ \mathrm{H} & 0.426624 & -0.745468 & 1.954098 \\ \mathrm{H} & 1.507387 & -0.774067 & 0.553064 \\ \mathrm{H} & -0.003395 & -1.707311 & 0.513280 \\ \mathrm{~N} & -0.181933 & 0.442588 & 0.337293 \\ \mathrm{C} & -0.249471 & 0.457793 & -1.118245 \\ \mathrm{H} & -1.124656 & 0.511251 & 0.714976 \\ \mathrm{H} & -0.785453 & 1.352463 & -1.452613 \\ \mathrm{H} & 0.767327 & 0.506805 & -1.526956 \\ \mathrm{H} & -0.742248 & -0.428527 & -1.563265 \\ & & & \\ \text { HF }=\mathbf{- 1 3 5 . 1 6 2 8 5 0 5} & & \end{array}$

Thermal correction to Enthalpy= 0.098306

Dimethylamine $\mathrm{N}$ radical:

\begin{tabular}{cccc}
\multicolumn{5}{c}{ Charge $=\mathbf{0}$ Multiplicity $=\mathbf{2}$} \\
C & 0.415686 & -0.719995 & 0.872599 \\
H & 0.415430 & -0.719557 & 1.967104 \\
H & 1.462065 & -0.764765 & 0.521253 \\
H & -0.068736 & -1.648567 & 0.521249
\end{tabular}




$\begin{array}{cccc}\mathrm{N} & -0.260362 & 0.450965 & 0.370223 \\ \mathrm{C} & -0.275576 & 0.477315 & -1.071900 \\ \mathrm{H} & -0.792429 & 1.372539 & -1.430716 \\ \mathrm{H} & 0.748084 & 0.471686 & -1.487322 \\ \mathrm{H} & -0.782539 & -0.412011 & -1.487321 \\ & & & \\ \mathbf{H F}=\mathbf{- 1 3 4 . 5 0 9 4 8 5 5} & & \end{array}$

Thermal correction to Enthalpy $=\mathbf{0 . 0 8 3 3 4 1}$

Methyleneamine:

Charge $=0$ Multiplicity $=1$

$\begin{array}{cccc}\mathrm{N} & -0.091131 & 0.000000 & -0.681785 \\ \mathrm{C} & -0.026019 & 0.000000 & 0.586857 \\ \mathrm{H} & 0.852160 & 0.000000 & -1.088402 \\ \mathrm{H} & 0.898970 & 0.000000 & 1.179349 \\ \mathrm{H} & -0.957095 & 0.000000 & 1.160404 \\ & & & \\ \mathbf{H F}=\mathbf{- 9 4 . 6 2 7 2 1 4 7} & & \end{array}$

Thermal correction to Enthalpy= 0.043918

Methyleneamine $\mathrm{N}$ radical:

Charge $=0$ Multiplicity $=2$

$\begin{array}{lrrr}\mathrm{N} & 0.000000 & 0.000000 & -0.743051 \\ \mathrm{C} & 0.000000 & 0.000000 & 0.505074 \\ \mathrm{H} & 0.935739 & 0.000000 & 1.085457 \\ \mathrm{H} & -0.935739 & 0.000000 & 1.085457\end{array}$

$\mathrm{HF}=\mathbf{- 9 3 . 9 8 2 4 4 2 6}$

Thermal correction to Enthalpy $=\mathbf{0 . 0 2 9 2 3 9}$

Aziridine:

Charge $=0$ Multiplicity $=1$

$\begin{array}{lrrr}\text { C } & -0.441045 & 0.037042 & -0.717383 \\ \text { N } & -0.441034 & 0.041172 & 0.754651 \\ \text { C } & 0.841486 & 0.037053 & 0.032162 \\ \text { H } & -0.804593 & 0.953701 & -1.176261 \\ \text { H } & 1.419701 & 0.953719 & 0.123731 \\ \text { H } & 1.439927 & -0.872872 & 0.020665 \\ \text { H } & -0.724719 & -0.872894 & -1.244427 \\ \text { H } & -0.645727 & -0.894428 & 1.105061 \\ & & & \\ \text { HF }=\mathbf{- 1 3 3 . 9 1 9 3 8 7 8} & & \end{array}$

Thermal correction to Enthalpy= $\mathbf{0 . 0 7 4 6 7 7}$ 
Aziridine $\mathbf{N}$ radical:

Charge $=0$ Multiplicity $=2$

$\begin{array}{lrrr}\mathrm{C} & -0.462535 & 0.000016 & -0.671365 \\ \mathrm{~N} & -0.464631 & 0.000004 & 0.792433 \\ \mathrm{C} & 0.811741 & -0.000016 & 0.075789 \\ \mathrm{H} & -0.819983 & 0.915381 & -1.143784 \\ \mathrm{H} & 1.398632 & 0.915297 & 0.157028 \\ \mathrm{H} & 1.398535 & -0.915395 & 0.157014 \\ \mathrm{H} & -0.820007 & -0.915314 & -1.143832\end{array}$

$\mathrm{HF}=\mathbf{- 1 3 3 . 2 6 8 3 2 3 3}$

Thermal correction to Enthalpy $=\mathbf{0 . 0 6 0 0 3 6}$

\section{Azetidine:}

Charge $=0$ Multiplicity $=1$

$\begin{array}{lrrr}\mathrm{C} & -0.734661 & 0.084827 & -0.757154 \\ \mathrm{C} & -0.735928 & 0.088008 & 0.791355 \\ \mathrm{C} & 0.808414 & 0.084789 & 0.677848 \\ \mathrm{~N} & 0.671777 & -0.387555 & -0.722379 \\ \mathrm{H} & -0.865591 & 1.098594 & -1.168521 \\ \mathrm{H} & -1.428683 & -0.591779 & -1.270300 \\ \mathrm{H} & -1.203039 & 0.938446 & 1.293668 \\ \mathrm{H} & -1.126343 & -0.842695 & 1.211149 \\ \mathrm{H} & 1.228215 & 1.098544 & 0.778641 \\ \mathrm{H} & 1.370502 & -0.591844 & 1.332835 \\ \mathrm{H} & 1.295547 & 0.057880 & -1.393114 \\ & & & \\ \text { HF }=\mathbf{- 1 7 3 . 2 3 7 7 1 2 5} & & \end{array}$

Thermal correction to Enthalpy= 0.105155

Azetidine $N$ radical:

Charge $=0$ Multiplicity $=2$

$\begin{array}{lrrr}\mathrm{C} & -0.703850 & 0.053055 & -0.767996 \\ \mathrm{C} & -0.704457 & 0.051463 & 0.779022 \\ \mathrm{C} & 0.834703 & 0.053056 & 0.623292 \\ \mathrm{~N} & 0.744288 & -0.240267 & -0.823069 \\ \mathrm{H} & -0.914618 & 1.039541 & -1.210530 \\ \mathrm{H} & -1.318356 & -0.684167 & -1.301668 \\ \mathrm{H} & -1.166147 & 0.901953 & 1.289580 \\ \mathrm{H} & -1.092720 & -0.876285 & 1.208383 \\ \mathrm{H} & 1.296141 & 1.039543 & 0.788623 \\ \mathrm{H} & 1.427305 & -0.684165 & 1.181189 \\ & & & \\ \mathbf{H F}=\mathbf{- 1 7 2 . 5 8 5 1 6 0 2} & & \end{array}$


Thermal correction to Enthalpy= 0.090448

Pyrrolidine:

Charge $=0$ Multiplicity $=1$

$\begin{array}{lrrr}\mathrm{C} & -0.953060 & -0.349832 & -0.798922 \\ \mathrm{C} & -0.950147 & -0.350088 & 0.744572 \\ \mathrm{~N} & 0.479711 & -0.358342 & 1.066052 \\ \mathrm{C} & 1.072703 & 0.633918 & 0.165097 \\ \mathrm{C} & 0.403164 & 0.310351 & -1.187498 \\ \mathrm{H} & 0.837923 & 1.675814 & 0.457243 \\ \mathrm{H} & 0.274487 & 1.210022 & -1.797153 \\ \mathrm{H} & -1.815520 & 0.192339 & -1.198743 \\ \mathrm{H} & -1.470561 & 0.552977 & 1.118298 \\ \mathrm{H} & -1.450687 & -1.224258 & 1.175601 \\ \mathrm{H} & 2.163494 & 0.533679 & 0.140085 \\ \mathrm{H} & 1.018663 & -0.389486 & -1.760662 \\ \mathrm{H} & -1.003013 & -1.373908 & -1.180896 \\ \mathrm{H} & 0.651268 & -0.134880 & 2.044361\end{array}$

$\mathrm{HF}=\mathbf{- 2 1 2 . 5 8 1 7 2 8 9}$

Thermal correction to Enthalpy $=\mathbf{0 . 1 3 6 1 6 4}$

Pyrrolidine $\mathbf{N}$ radical:

Charge $=0$ Multiplicity $=2$

$\begin{array}{lrrr}\mathrm{C} & -0.965901 & -0.208509 & -0.763561 \\ \mathrm{C} & -0.964895 & -0.206800 & 0.780252 \\ \mathrm{~N} & 0.415234 & -0.208401 & 1.245850 \\ \mathrm{C} & 1.253538 & 0.062046 & 0.085857 \\ \mathrm{C} & 0.351064 & 0.516957 & -1.081208 \\ \mathrm{H} & 2.040887 & 0.778861 & 0.354905 \\ \mathrm{H} & 0.206831 & 1.604023 & -1.043476 \\ \mathrm{H} & -1.848014 & 0.275108 & -1.195028 \\ \mathrm{H} & -1.441725 & 0.710104 & 1.171421 \\ \mathrm{H} & -1.511521 & -1.044318 & 1.233991 \\ \mathrm{H} & 1.776776 & -0.877900 & -0.166753 \\ \mathrm{H} & 0.761319 & 0.269974 & -2.065517 \\ \mathrm{H} & -\mathbf{- 0 . 9 3 4 0 2 1} & -1.239202 & -1.138532 \\ & & & \\ \text { HF= -211.9338792 } & & \end{array}$

Thermal correction to Enthalpy $=\mathbf{0 . 1 2 1 4 2 2}$

Piperidine:

Charge $=\mathbf{0}$ Multiplicity $=1$

$\begin{array}{llll}\text { C } & -1.048931 & -0.638930 & -0.760292\end{array}$ 


$\begin{array}{lrrr}\mathrm{N} & -1.054380 & -0.631194 & 0.704506 \\ \mathrm{C} & 0.300981 & -0.638735 & 1.260061 \\ \mathrm{C} & 1.042363 & 0.630150 & 0.824570 \\ \mathrm{C} & 1.059221 & 0.749299 & -0.707609 \\ \mathrm{C} & -0.362516 & 0.629798 & -1.278941 \\ \mathrm{H} & 0.227409 & -0.681754 & 2.353220 \\ \mathrm{H} & 0.529713 & 1.499548 & 1.256227 \\ \mathrm{H} & 2.064827 & 0.619209 & 1.223027 \\ \mathrm{H} & 1.520085 & 1.696268 & -1.015556 \\ \mathrm{H} & 1.684145 & -0.054543 & -1.124824 \\ \mathrm{H} & -0.957589 & 1.499395 & -0.971191 \\ \mathrm{H} & -0.338879 & 0.618275 & -2.376022 \\ \mathrm{H} & -2.087031 & -0.681652 & -1.110685 \\ \mathrm{H} & -0.525620 & -1.524143 & -1.177669 \\ \mathrm{H} & 0.887094 & -1.523692 & 0.936065 \\ \mathrm{H} & -1.570204 & -1.438045 & 1.049132 \\ & & & \\ \text { HF }=\mathbf{- 2 5 1 . 9 0 4 3 9 8 8} & & \\ \text { Thermal correction to Enthalpy }=\mathbf{0 . 1 6 6 4 5 6}\end{array}$

Piperidine N radical:

Charge $=0$ Multiplicity $=2$

\begin{tabular}{lrrr}
$\mathrm{C}$ & -1.091795 & -0.665124 & -0.695864 \\
$\mathrm{~N}$ & -1.096411 & -0.663909 & 0.752587 \\
$\mathrm{C}$ & 0.256872 & -0.665134 & 1.268955 \\
$\mathrm{C}$ & 1.029279 & 0.600683 & 0.821255 \\
$\mathrm{C}$ & 1.031675 & 0.717078 & -0.708150 \\
$\mathrm{C}$ & -0.396362 & 0.600698 & -1.255688 \\
$\mathrm{H}$ & 0.222345 & -0.723473 & 2.362704 \\
$\mathrm{H}$ & 0.542416 & 1.481015 & 1.260335 \\
$\mathrm{H}$ & 2.050310 & 0.563736 & 1.220742 \\
$\mathrm{H}$ & 1.484854 & 1.666898 & -1.019214 \\
$\mathrm{H}$ & 1.652025 & -0.084668 & -1.133973 \\
$\mathrm{H}$ & -0.981104 & 1.481027 & -0.959190 \\
$\mathrm{H}$ & -0.402116 & 0.563773 & -2.352075 \\
$\mathrm{H}$ & -2.124846 & -0.723455 & -1.056812 \\
$\mathrm{H}$ & -0.552598 & -1.553343 & -1.076177 \\
$\mathrm{H}$ & 0.805586 & -1.553354 & 0.902506 \\
& & & \\
HF $=\mathbf{- 2 5 1 . 2 5 0 3 2 5 2}$ & & \\
\hline
\end{tabular}

Thermal correction to Enthalpy= $\mathbf{0 . 1 5 2 2 5 9}$

Azepane:

Charge $=0$ Multiplicity $=1$ 


$\begin{array}{lrrr}\mathrm{C} & -1.095366 & 0.920441 & -0.726453 \\ \mathrm{C} & -1.074975 & 0.916698 & 0.814495 \\ \mathrm{C} & 0.315923 & 0.890237 & 1.483123 \\ \mathrm{C} & 1.406992 & 0.040445 & 0.786514 \\ \mathrm{~N} & 1.013583 & -1.224365 & 0.167215 \\ \mathrm{C} & 0.451564 & -1.130279 & -1.179897 \\ \mathrm{C} & -0.935038 & -0.479905 & -1.344820 \\ \mathrm{H} & -0.325073 & 1.601531 & -1.119727 \\ \mathrm{H} & -1.698006 & -1.141153 & -0.904662 \\ \mathrm{H} & 1.183025 & -0.576084 & -1.785429 \\ \mathrm{H} & -1.661043 & 0.050978 & 1.155874 \\ \mathrm{H} & 0.190142 & 0.540975 & 2.518101 \\ \mathrm{H} & -2.058151 & 1.330445 & -1.060811 \\ \mathrm{H} & -1.157054 & -0.434255 & -2.422078 \\ \mathrm{H} & 0.415701 & -2.145775 & -1.597464 \\ \mathrm{H} & -1.610982 & 1.798952 & 1.189738 \\ \mathrm{H} & 0.705100 & 1.916313 & 1.562293 \\ \mathrm{H} & 2.199029 & -0.165229 & 1.520408 \\ \mathrm{H} & 1.880273 & 0.632691 & -0.006860 \\ \mathrm{H} & 0.427352 & -1.784661 & 0.782338 \\ & & & \\ \text { HF= -291.2178715 } & & \\ \mathrm{T} & & & \end{array}$

Thermal correction to Enthalpy $=\mathbf{0 . 1 9 5 8 8 3}$

\begin{tabular}{|c|c|c|c|}
\hline \multicolumn{4}{|c|}{$\begin{array}{l}\text { Azepane } N \text { radical: } \\
\text { Charge }=0 \text { Multiplicity }=2\end{array}$} \\
\hline & -1.045789 & 0.897600 & -0.733345 \\
\hline $\mathrm{C}$ & -1.044427 & 0.922198 & 0.809535 \\
\hline $\mathrm{C}$ & 0.336120 & 0.836310 & 1.498165 \\
\hline $\mathrm{C}$ & 1.407709 & -0.001370 & 0.746580 \\
\hline $\mathrm{N}$ & 0.862390 & -1.247062 & 0.260938 \\
\hline $\mathrm{C}$ & 0.490208 & -1.184636 & -1.132555 \\
\hline $\mathrm{C}$ & -0.893845 & -0.511470 & -1.333681 \\
\hline $\mathrm{H}$ & -0.270967 & 1.568880 & -1.133010 \\
\hline $\mathrm{H}$ & -1.665123 & -1.164132 & -0.903405 \\
\hline $\mathrm{H}$ & 1.239482 & -0.624350 & -1.722116 \\
\hline $\mathrm{H}$ & -1.671149 & 0.090468 & 1.158063 \\
\hline $\mathrm{H}$ & 0.203607 & 0.421866 & 2.504217 \\
\hline $\mathrm{H}$ & -2.004929 & 1.303597 & -1.081998 \\
\hline $\mathrm{H}$ & -1.076115 & -0.475778 & -2.416508 \\
\hline $\mathrm{H}$ & 0.435993 & -2.205308 & -1.531094 \\
\hline $\mathrm{H}$ & -1.539926 & 1.838881 & 1.155519 \\
\hline $\mathrm{H}$ & 0.757926 & 1.842800 & 1.624161 \\
\hline $\mathrm{H}$ & 2.214446 & -0.228046 & 1.457018 \\
\hline
\end{tabular}


$\begin{array}{llll}\mathrm{H} & 1.840171 & 0.608756 & -0.065599\end{array}$

$\mathrm{HF}=\mathbf{- 2 9 0 . 5 6 6 0 5 7 8}$

Thermal correction to Enthalpy $=\mathbf{0 . 1 8 2 2 1 8}$

2-Aza-bicyclo[1.1.0]butane:

Charge $=0$ Multiplicity $=1$

$\begin{array}{lrrr}\mathrm{C} & -0.561590 & 0.248439 & -0.975745 \\ \mathrm{C} & -0.560396 & 0.220521 & 0.526146 \\ \mathrm{~N} & 0.881237 & 0.190781 & 0.781282 \\ \mathrm{C} & 0.287614 & -0.718586 & -0.201037 \\ \mathrm{H} & -0.081075 & 1.084723 & -1.495391 \\ \mathrm{H} & -1.440439 & -0.150011 & -1.486050 \\ \mathrm{H} & 1.342706 & 0.975940 & 0.305449 \\ \mathrm{H} & 0.341222 & -1.796704 & -0.158923 \\ \mathrm{H} & -1.324845 & 0.048338 & 1.269758 \\ & & & \\ \text { HF }=\mathbf{- 1 7 1 . 9 6 5 6 6 9 9} & & \end{array}$

Thermal correction to Enthalpy= 0.079754

2-Aza-bicyclo[1.1.0]butane $\mathrm{N}$ radical:

Charge $=0$ Multiplicity $=2$

$\begin{array}{cccc}\mathrm{C} & -0.521752 & 0.268887 & -0.955694 \\ \mathrm{C} & -0.503988 & 0.299679 & 0.543522 \\ \mathrm{~N} & 0.893065 & 0.185756 & 0.801563 \\ \mathrm{C} & 0.375188 & -0.673942 & -0.210387 \\ \mathrm{H} & -0.057642 & 1.100587 & -1.488551 \\ \mathrm{H} & -1.403872 & -0.149116 & -1.444562 \\ \mathrm{H} & 0.412298 & -1.757277 & -0.204938 \\ \mathrm{H} & -1.298926 & 0.137774 & 1.262465\end{array}$

$\mathrm{HF}=\mathbf{- 1 7 1 . 3 2 9 7 7 5 1}$

Thermal correction to Enthalpy $=\mathbf{0 . 0 6 6 0 4 1}$

5-Aza-bicyclo[2.1.0]pentane:

Charge $=0$ Multiplicity $=1$

$\begin{array}{lrrr}\mathrm{C} & -0.652509 & -0.610102 & -0.374270 \\ \mathrm{~N} & -0.641337 & -0.647821 & 1.083570 \\ \mathrm{C} & 0.642090 & -0.610011 & 0.392025 \\ \mathrm{C} & 0.976786 & 0.803082 & -0.112047 \\ \mathrm{C} & -0.371770 & 0.802981 & -0.910282 \\ \mathrm{H} & 1.846780 & 0.832861 & -0.774927 \\ \mathrm{H} & 1.104710 & 1.566424 & 0.665793 \\ \mathrm{H} & -1.115306 & 1.566274 & -0.648314\end{array}$




$\begin{array}{rrrr}\mathrm{H} & -0.209157 & 0.832671 & -1.991885 \\ \mathrm{H} & -1.160475 & -1.414268 & -0.899387 \\ \mathrm{H} & 1.346905 & -1.414090 & 0.584771 \\ \mathrm{H} & -0.891677 & 0.249173 & 1.506400 \\ & & & \\ \mathbf{H F}=\mathbf{- 2 1 1 . 3 0 9 5 9 6 5} & & \end{array}$

Thermal correction to Enthalpy $=\mathbf{0 . 1 1 0 6 3 8}$

5-Aza-bicyclo[2.1.0]pentane $\mathrm{N}$ radical:

Charge $=\mathbf{0}$ Multiplicity $=2$

$\begin{array}{lrrr}\text { C } & -0.686379 & -0.605454 & -0.340300 \\ \text { N } & -0.650449 & -0.650293 & 1.098831 \\ \text { C } & 0.628576 & -0.605338 & 0.438157 \\ \text { C } & 0.945947 & 0.813109 & -0.066290 \\ \text { C } & -0.397014 & 0.813017 & -0.861234 \\ \text { H } & 1.820073 & 0.840706 & -0.722546 \\ \text { H } & 1.061545 & 1.567312 & 0.719134 \\ \text { H } & -1.141298 & 1.567133 & -0.584774 \\ \text { H } & -0.242181 & 0.840595 & -1.943266 \\ \text { H } & -1.247974 & -1.377964 & -0.862019 \\ \text { H } & 1.356196 & -1.377738 & 0.679654 \\ & & & \\ \text { HF }=\mathbf{- 2 1 0 . 6 5 8 8 4 7} & & \\ \text { Thermal correction to Enthalpy= } & \mathbf{0 . 0 9 6 6 8 2}\end{array}$

Thermal correction to Enthalpy= $\mathbf{0 . 0 9 6 6 8 2}$

\begin{tabular}{|c|c|c|c|}
\hline & \\
\hline \multicolumn{4}{|c|}{$\begin{array}{l}\text { 6-Aza-bicyclo[3.1.0]hexane: } \\
\text { Charge=0 Multiplicity=1 } \\
\text { N } \quad-0.322329 \quad 0.776969\end{array}$} \\
\hline \multicolumn{4}{|c|}{$\begin{array}{lll}\mathrm{N} & -0.322329 & 0.776969 \\
\mathrm{C} & -0.728387 & 0.857966\end{array}$} \\
\hline $\mathrm{C}$ & 0.442330 & 0.729462 & 1.120738 \\
\hline $\mathrm{C}$ & 1.235914 & -0.489258 & 0.583308 \\
\hline \multirow{2}{*}{$\begin{array}{l}\mathrm{C} \\
\mathrm{C}\end{array}$} & 0.187606 & -1.390908 & -0.118091 \\
\hline & -0.881639 & -0.417398 & -0.583971 \\
\hline $\mathrm{H}$ & 1.781063 & -1.016111 & 1.372966 \\
\hline $\mathrm{H}$ & 1.966235 & -0.146350 & -0.153796 \\
\hline \multirow{2}{*}{$\begin{array}{l}\mathrm{H} \\
\mathrm{H}\end{array}$} & 1.057496 & 1.635370 & 1.171034 \\
\hline & 0.052475 & 0.534706 & 2.128992 \\
\hline $\mathrm{H}$ & -1.586706 & 1.471602 & 0.426746 \\
\hline \multirow{2}{*}{$\begin{array}{l}\mathrm{H} \\
\mathrm{H}\end{array}$} & -1.857964 & -0.785833 & -0.892007 \\
\hline & -0.263804 & -2.098271 & 0.590675 \\
\hline $\mathrm{H}$ & 0.623913 & -1.973711 & -0.937611 \\
\hline $\mathrm{H}$ & -1.051345 & 1.200634 & -1.839317 \\
\hline
\end{tabular}


Thermal correction to Enthalpy=0.141683

\begin{tabular}{|c|c|c|c|}
\hline \\
\hline \multicolumn{2}{|c|}{$\begin{array}{llll}C & -0.860706 & -0.250114 & -0.741947\end{array}$} & \multicolumn{2}{|c|}{$\begin{array}{l}\text { 6-Aza-bicyclo[3.1.0]hexane } \mathbf{N} \text { radical: } \\
\text { Charge=0 Multiplicity=2 }\end{array}$} \\
\hline \multicolumn{4}{|c|}{$\begin{array}{llll}\mathrm{C} & -0.860710 & -0.250116 & 0.741941\end{array}$} \\
\hline \\
\hline \multirow{2}{*}{$\begin{array}{l}\mathrm{C} \\
\mathrm{C}\end{array}$} & & & 004 \\
\hline & 0.58 & -0.24 & -1.232980 \\
\hline \multirow{2}{*}{$\begin{array}{l}\mathrm{N} \\
\mathrm{H}\end{array}$} & -1.279592 & 0.941 & -0.000003 \\
\hline & & & 007 \\
\hline \multirow{2}{*}{$\begin{array}{l}\mathrm{H} \\
\mathrm{H}\end{array}$} & 1.475197 & 1.00 & 0.000006 \\
\hline & 0.733576 & 0.41 & 2.097392 \\
\hline \multirow{2}{*}{$\begin{array}{l}\mathrm{H} \\
\mathrm{H}\end{array}$} & & & 758 \\
\hline & -1.628525 & -0.712247 & 1.361957 \\
\hline \multirow{2}{*}{$\begin{array}{l}\mathrm{H} \\
\mathrm{H}\end{array}$} & -1.628517 & -0.712244 & -1.361968 \\
\hline & 0.858716 & -1.259668 & -1.539756 \\
\hline $\mathrm{H}$ & 0.733588 & 0.416547 & -2.097387 \\
\hline
\end{tabular}

$\mathrm{HF}=\mathbf{- 2 5 0 . 0 0 9 3 8 5 4}$

Thermal correction to Enthalpy $=\mathbf{0 . 1 2 7 7 2 1}$

2-Aza-bicyclo[1.1.1]pentane:

Charge $=0$ Multiplicity $=1$

$\begin{array}{lrrr}\mathrm{C} & -0.562448 & 0.504876 & -0.996223 \\ \mathrm{C} & -0.542868 & 0.482966 & 0.552484 \\ \mathrm{C} & 1.009381 & 0.512490 & 0.494957 \\ \mathrm{C} & 0.508040 & -0.521971 & -0.550130 \\ \mathrm{~N} & -0.526756 & -1.038299 & 0.444265 \\ \mathrm{H} & 1.093968 & -1.204100 & -1.172910 \\ \mathrm{H} & -1.211656 & 1.000666 & 1.246153 \\ \mathrm{H} & 1.530518 & 0.100273 & 1.367356 \\ \mathrm{H} & 1.499512 & 1.424286 & 0.131081 \\ \mathrm{H} & -0.209040 & 1.414846 & -1.488748 \\ \mathrm{H} & -1.455179 & 0.083622 & -1.463154 \\ \mathrm{H} & -0.033457 & -1.421663 & 1.263835 \\ & & & \\ \text { HF }=\mathbf{- 2 1 1 . 2 7 9 5 2 7 2} & & \\ \text { Thermal corretion }\end{array}$

Thermal correction to Enthalpy= $\mathbf{0 . 1 1 0 7 5 1}$

2-Aza-bicyclo[1.1.1]pentane $\mathrm{N}$ radical:

Charge $=\mathbf{0}$ Multiplicity $=2$
C $\quad-0.575193$
$0.461184 \quad-0.968549$
C -0.557473
0.445720
0.584889 


$\begin{array}{rrrr}\mathrm{C} & 0.995035 & 0.460948 & 0.528260 \\ \mathrm{C} & 0.502050 & -0.567454 & -0.526764 \\ \mathrm{~N} & -0.477474 & -1.048712 & 0.500726 \\ \mathrm{H} & 1.086507 & -1.256782 & -1.139997 \\ \mathrm{H} & -1.224679 & 0.953300 & 1.284902 \\ \mathrm{H} & 1.511694 & 0.047358 & 1.397641 \\ \mathrm{H} & 1.473522 & 1.373323 & 0.152760 \\ \mathrm{H} & -0.222872 & 1.373579 & -1.464318 \\ \mathrm{H} & -1.468365 & 0.047813 & -1.443085 \\ & & & \\ \text { HF= -210.6362589 } & & \end{array}$

Thermal correction to Enthalpy $=\mathbf{0 . 0 9 7 1 0 2}$

\section{2-Aza-tricyclo[1.1.1.01,3]pentane:}

Charge $=0$ Multiplicity $=1$

$\begin{array}{cccc}\mathrm{C} & -0.420319 & 0.531678 & -1.110993 \\ \mathrm{C} & -0.426744 & 0.509019 & 0.404769 \\ \mathrm{~N} & 1.058034 & 0.549683 & 0.492158 \\ \mathrm{C} & 0.462149 & -0.478006 & -0.403858 \\ \mathrm{C} & -0.587418 & -0.992741 & 0.566071 \\ \mathrm{H} & 1.353709 & 0.100330 & 1.365664 \\ \mathrm{H} & 0.086951 & 1.370277 & -1.576984 \\ \mathrm{H} & -1.302616 & 0.156880 & -1.623391 \\ \mathrm{H} & -0.228976 & -1.431187 & 1.495259 \\ \mathrm{H} & -1.481316 & -1.463781 & 0.158404\end{array}$

$\mathrm{HF}=\mathbf{- 2 1 0 . 0 2 6 6 6 4 6}$

Thermal correction to Enthalpy=0.086575

2-Aza-tricyclo[1.1.1.01,3]pentane $\mathrm{N}$ radical: Charge $=\mathbf{0}$ Multiplicity $=2$

$\begin{array}{cccc}\mathrm{C} & -0.376405 & 0.546583 & -1.080886 \\ \mathrm{C} & -0.393450 & 0.524434 & 0.437116 \\ \mathrm{~N} & 1.078026 & 0.509555 & 0.563096 \\ \mathrm{C} & 0.504097 & -0.472219 & -0.379400 \\ \mathrm{C} & -0.536094 & -0.977835 & 0.604308 \\ \mathrm{H} & 0.123636 & 1.385719 & -1.555489 \\ \mathrm{H} & -1.260282 & 0.172113 & -1.595396 \\ \mathrm{H} & -0.168914 & -1.407081 & 1.531867 \\ \mathrm{H} & -1.429514 & -1.443416 & 0.190520 \\ & & & \\ \mathbf{H F}=\mathbf{- 2 0 9 . 3 7 4 9 3 8 3} & & \end{array}$

Thermal correction to Enthalpy=0.072498 


\section{5-Aza-bicyclo[2.1.1]hexane:}

\section{Charge $=0$ Multiplicity $=1$}

$\begin{array}{lrrr}\mathrm{N} & -0.705552 & 0.632102 & -0.971345 \\ \mathrm{C} & -0.659247 & 0.653994 & 0.529074 \\ \mathrm{C} & 0.850978 & 0.670403 & 0.873505 \\ \mathrm{C} & 1.322177 & -0.401261 & -0.165533 \\ \mathrm{C} & -0.058581 & -0.711332 & -0.795523 \\ \mathrm{C} & -0.971221 & -0.862407 & 0.448448 \\ \mathrm{H} & -1.312730 & 1.363816 & 1.042758 \\ \mathrm{H} & 1.056507 & 0.383729 & 1.910837 \\ \mathrm{H} & 1.298048 & 1.656530 & 0.703594 \\ \mathrm{H} & 1.786683 & -1.277467 & 0.300310 \\ \mathrm{H} & 2.027006 & -0.000920 & -0.903183 \\ \mathrm{H} & -0.095620 & -1.402717 & -1.641319 \\ \mathrm{H} & -2.010323 & -1.058169 & 0.178833 \\ \mathrm{H} & -0.637566 & -1.508676 & 1.265938 \\ \mathrm{H} & -0.077773 & 1.322778 & -1.398178 \\ & & & \\ \text { HF= -250.6462968 } & & \end{array}$

Thermal correction to Enthalpy= 0.141951

5-Aza-bicyclo[2.1.1]hexane $\mathrm{N}$ radical:

Charge $=0$ Multiplicity $=2$

$\begin{array}{lrrr}\text { C } & -0.675075 & -0.630787 & -0.996027 \\ \mathrm{C} & -0.665453 & -0.633401 & 0.557436 \\ \mathrm{C} & 0.840926 & -0.622975 & 0.901226 \\ \mathrm{C} & 1.288003 & 0.487273 & -0.105071 \\ \mathrm{C} & -0.094384 & 0.784909 & -0.727854 \\ \mathrm{~N} & -0.996902 & 0.818389 & 0.460187 \\ \mathrm{H} & -1.327268 & -1.255902 & 1.163249 \\ \mathrm{H} & 1.313703 & -1.594446 & 0.720923 \\ \mathrm{H} & 1.017765 & -0.342274 & 1.943709 \\ \mathrm{H} & 2.011492 & 0.138534 & -0.849879 \\ \mathrm{H} & 1.705676 & 1.365777 & 0.395536 \\ \mathrm{H} & -0.172377 & 1.612468 & -1.436075 \\ \mathrm{H} & -1.683862 & -0.600989 & -1.411423 \\ \mathrm{H} & -0.050913 & -1.362009 & -1.525606 \\ & & & \\ \text { HF }=\mathbf{- 2 4 9 . 9 9 4 8 9 5 3} & & \end{array}$

Thermal correction to Enthalpy $=\mathbf{0 . 1 2 8 1 7 3}$

5-Aza-tricyclo[2.1.1.01,4]hexane:

Charge $=0$ Multiplicity $=1$

$\begin{array}{llll}\mathrm{N} & -0.420442 & 0.628367 & -1.206100\end{array}$ 


$\begin{array}{cccc}\mathrm{C} & -0.549529 & 0.653184 & 0.254039 \\ \mathrm{C} & 0.885443 & 0.692834 & 0.857456 \\ \mathrm{C} & 1.297191 & -0.559437 & 0.060323 \\ \mathrm{C} & -0.116831 & -0.662951 & -0.582342 \\ \mathrm{C} & -1.230548 & -0.680397 & 0.434627 \\ \mathrm{H} & 0.882702 & 0.575481 & 1.943970 \\ \mathrm{H} & 1.407530 & 1.616105 & 0.600096 \\ \mathrm{H} & 1.545938 & -1.436697 & 0.662671 \\ \mathrm{H} & 2.073818 & -0.412159 & -0.692303 \\ \mathrm{H} & -2.241755 & -0.758862 & 0.035010 \\ \mathrm{H} & -1.086164 & -1.226241 & 1.368227 \\ \mathrm{H} & -1.353327 & 0.584409 & -1.619596 \\ & & & \\ \mathbf{H F}=\mathbf{- 2 4 9 . 3 4 4 1 4 1 2} & & \end{array}$

Thermal correction to Enthalpy $=\mathbf{0 . 1 1 6 5 6 0}$

\section{5-Aza-tricyclo[2.1.1.01,4]hexane $\mathrm{N}$ radical:}

Charge $=\mathbf{0}$ Multiplicity $=\mathbf{2}$

$\begin{array}{lrcc}\mathrm{N} & -0.499968 & 0.666718 & -1.198024 \\ \mathrm{C} & -0.576738 & 0.663828 & 0.237645 \\ \mathrm{C} & 0.875119 & 0.689879 & 0.818832 \\ \mathrm{C} & 1.264063 & -0.559123 & -0.037210 \\ \mathrm{C} & -0.176666 & -0.620959 & -0.644622 \\ \mathrm{C} & -1.235084 & -0.676694 & 0.425722 \\ \mathrm{H} & 0.912005 & 0.555698 & 1.902637 \\ \mathrm{H} & 1.412227 & 1.601913 & 0.549242 \\ \mathrm{H} & 1.535852 & -1.455890 & 0.524927 \\ \mathrm{H} & 2.025789 & -0.363547 & -0.795156 \\ \mathrm{H} & -2.256828 & -0.746727 & 0.064401 \\ \mathrm{H} & -1.033423 & -1.240062 & 1.337907 \\ & & & \\ \text { HF }=\mathbf{- 2 4 8 . 6 9 9 4 5 2 3} & & \end{array}$

Thermal correction to Enthalpy= $\mathbf{0 . 1 0 2 7 1 7}$ 